\title{
Symmetric homology of algebras
}

\author{
SHAUN V AULT
}

\begin{abstract}
The symmetric homology of a unital algebra $A$ over a commutative ground ring $k$ is defined using derived functors and the symmetric bar construction of Fiedorowicz. For a group ring $A=k[\Gamma]$, the symmetric homology is related to stable homotopy theory via $H S_{*}(k[\Gamma]) \cong H_{*}\left(\Omega \Omega^{\infty} S^{\infty}(B \Gamma) ; k\right)$. Two chain complexes that compute $H S_{*}(A)$ are constructed, both making use of a symmetric monoidal category $\Delta S_{+}$containing $\Delta S$. Two spectral sequences are found that aid in computing symmetric homology. The second spectral sequence is defined in terms of a family of complexes, $\operatorname{Sym}_{*}^{(p)}$. $\operatorname{Sym}^{(p)}$ is isomorphic to the suspension of the cycle-free chessboard complex $\Omega_{p+1}$ of Vrećica and Živaljević, and so recent results on the connectivity of $\Omega_{n}$ imply finite-dimensionality of the symmetric homology groups of finite-dimensional algebras. Some results about the $k \Sigma_{p+1}$-module structure of $\operatorname{Sym}^{(p)}$ are devloped. A partial resolution is found that allows computation of $H S_{1}(A)$ for finite-dimensional $A$ and some concrete computations are included.
\end{abstract}

\section{Introduction and definitions}

The theory of symmetric homology, in which the symmetric groups $\Sigma_{k}^{\text {op }}$, for $k \geq 0$, play the role that the cyclic groups do in cyclic homology, begins with the definition of the category $\Delta S$, containing the simplicial category $\Delta$ as subcategory. Indeed, $\Delta S$ is an example of crossed simplicial group (see Fiedorowicz and Loday [10]). One develops a notion of a bar resolution over crossed simplicial groups by analogy with the cyclic bar resolution. However, the naïve contravariant symmetric bar resolution produces trivial results. Fiedorowicz found that a covariant symmetric bar construction, $B_{*}^{\text {sym }}$, produces a non-trivial and important homology theory (see Fiedorowicz [8]). One defines the symmetric homology of an algebra $A$ by

Definition $1 H S_{*}(A)=\operatorname{Tor}_{*}^{\Delta S}\left(\underline{k}, B_{*}^{\mathrm{sym}} A\right)$.

Almost 20 years ago, Fiedorowicz found but did not publish the following remarkable result [8]. 
Theorem 2 (Fiedorowicz's Theorem) If $\Gamma$ is a group, then

$$
H S_{*}(k[\Gamma]) \cong H_{*}\left(\Omega \Omega^{\infty} S^{\infty}(B \Gamma) ; k\right) .
$$

This formula shows in particular that $H S_{*}$ is an important and non-trivial theory. While it is true that $H_{*}\left(\Omega^{\infty} S^{\infty} X\right)=H_{*}(Q X)$ is well understood, the same cannot be said of the homology of $\Omega \Omega^{\infty} S^{\infty} X$. Indeed, $H_{*}(Q X)$ has been studied extensively by Cohen, Lada and May [4]. $H_{*}(Q X)$ may be regarded as the free allowable $A R$-Hopf algebra with conjugation generated by $H_{*}(X)$. On the other hand, results about the homology of $\Omega \Omega^{\infty} S^{\infty} X$ are fewer and farther between. Cohen and Peterson [5] computed $H_{*}\left(\Omega \Omega^{\infty} S^{\infty}\right)$ (that is, the case when the space $X$ is the zero-sphere, $\left.S^{0}\right)$, but there is little hope of extending this result to arbitrary $X$ using the same methods.

We find that adjoining a "unit" to $\Delta S$ results in a permutative category $\Delta S_{+}$. This step is necessary in order to prove Theorem 2 and related theorems.

By reducing the standard resolution that computes $H S_{*}(A)$ to one that involves only epimorphisms of $\Delta S_{+}$, we develop two spectral sequences abutting to $H S_{*}(A)$. The first spectral sequence is based on the work of Słomińska [27] on $E-I$-categories, and relates symmetric homology to the homology of the symmetric groups.

The second spectral sequence makes use of a family of complexes, $\operatorname{Sym}_{*}^{(p)}$ which distill the relevent combinatorial data of the nerve of $\operatorname{Epi}_{\Delta S}$.

Theorem 3 If $A$ has an augmentation ideal $I$ which is free as $k$-module, with countable basis $X$, then there is a spectral sequence $E_{p, q}^{1} \Rightarrow \widetilde{H} S_{p+q}(A)$, with

$$
E_{p, q}^{1} \cong \bigoplus_{u \in X^{p+1 / \Sigma_{p+1}}} H_{p+q}\left(E_{*} G_{u} \otimes_{G_{u}} \operatorname{Sym}_{*}^{(p)} ; k\right),
$$

where $G_{u}$ is the isotropy subgroup for the chosen representative of $u \in X^{p+1} / \Sigma_{p+1}$ and the symbol $\otimes_{G_{u}}$ stands for the chain complex analog of equivariant half-smash product for spaces, $\ltimes_{G_{u}}$.

The multiplicative structure of $A$ becomes encapsulated in the differential $d_{p, q}^{1}$ on $E^{1}$, and so results about $H S_{*}(A)$ for general algebras $A$ will follow from the structure of $\operatorname{Sym}_{*}^{(p)}$. There is an isomorphism of complexes, $k\left[S \Omega_{p+1}^{+}\right] \stackrel{\cong}{\longrightarrow} \operatorname{Sym}_{*}^{(p)}$, where $\Omega_{n}^{+}$ is the augmented cycle-free $(n \times n)$-chessboard complex of Vrećica and Živaljević [30], which produces the following immediate important corollary:

Corollary 4 If $A$ is finitely-generated over a Noetherian ground ring $k$, then $H S_{*}(A)$ is finitely-generated over $k$ in each degree. 
In the final section, we develop a partial resolution of the trivial $\Delta S^{\mathrm{op}}$-module $\underline{k}$ by projective modules, leading to the following:

Theorem $5 H S_{i}(A)$ for $i=0,1$ may be computed as the degree 0 and degree 1 homology groups of the (partial) chain complex

$$
0 \longleftarrow A \stackrel{\partial_{1}}{\longleftarrow} A \otimes A \otimes A \stackrel{\partial_{2}}{\longleftarrow}(A \otimes A \otimes A \otimes A) \oplus A,
$$

where

$$
\begin{aligned}
& \partial_{1}: a \otimes b \otimes c \mapsto a b c-c b a, \\
& \partial_{2}:\left\{\begin{array}{cc}
a \otimes b \otimes c \otimes d & \mapsto a b \otimes c \otimes d+d \otimes c a \otimes b \\
a & +b c a \otimes 1 \otimes d+d \otimes b c \otimes a, \\
& \mapsto 1 \otimes a \otimes 1 .
\end{array}\right.
\end{aligned}
$$

In particular, we see that $H S_{0}(A)=A /([A, A])$, where $([A, A])$ is the ideal generated by the commutator submodule $[A, A]$. In other words, $H S_{0}(A)$ is the symmetrization of $A$ as an algebra. Compare with the zeroth Hochschild or Cyclic homology of $A$, which is $A /[A, A]$.

\subsection{The category $\Delta S$}

Let $\Delta S$ be the category that has as objects, the ordered sets $[n]=\{0,1, \ldots, n\}$ for $n \geq 0$, and as morphisms, pairs $(\phi, g)$, where $\phi:[n] \rightarrow[m]$ is a non-decreasing map of sets (that is, a morphism in $\Delta$ ), and $g \in \Sigma_{n+1}^{\mathrm{op}}$ (the opposite group of the symmetric group acting on $[n]$ ). The element $g$ represents an automorphism of $[n]$, and as a set map, takes $i \in[n]$ to $g^{-1}(i)$. Equivalently, a morphism in $\Delta S$ is a morphism in $\Delta$ together with a total ordering of the domain $[n]$. Composition of morphisms is achieved as in Fiedorowicz-Loday [10], namely, $(\phi, g) \circ(\psi, h)=\left(\phi \cdot g^{*}(\psi), \psi^{*}(g) \cdot h\right)$. Observe that the properties of $g^{*}(\phi)$ and $\phi^{*}(g)$ stated in [10, Proposition 1.6] are formally rather similar to the properties of exponents (except for properties $2 . \mathrm{h}$ and 2.v). Indeed, the notation $g \stackrel{\phi^{\text {def }}}{=} \phi^{*}(g), \phi^{g} \stackrel{\text { def }}{=} g^{*}(\phi)$ will generally be used in lieu of the original notation in what follows. For reference, we restate [10, Proposition 1.6] using the "exponent" notation:

Proposition 6 If $G_{*}$ is a crossed simplicial group, for $g, h \in G_{n}, \phi \in \operatorname{Mor}_{\Delta}([m],[n])$, $\psi \in \operatorname{Mor}_{\Delta}([p],[m])$,
$(1 . h)^{\prime} \quad g^{\phi \psi}=\left(g^{\phi}\right)^{\psi}$
$(2 . h)^{\prime} \quad(\phi \psi)^{g}=\phi^{g} \psi^{\left(g^{\phi}\right)}$
$(1 . v)^{\prime} \quad \phi^{g h}=\left(\phi^{g}\right)^{h}$
(3.h) $)^{\prime} \quad g^{\text {id }_{n}}=g \quad$ and $\quad 1^{\phi}=1$
$(2 . v)^{\prime} \quad(g h)^{\phi}=g^{\phi} h^{\left(\phi^{g}\right)}$
(3.v) $)^{\prime} \quad \phi^{1}=\phi \quad$ and $\quad \mathrm{id}_{n}^{g}=\mathrm{id}$ 
It is often helpful to represent morphisms of $\Delta S$ as diagrams of points and lines, indicating images of set maps. Using these diagrams, we may see clearly how $\psi^{g}$ and $g^{\psi}$ are related to $\psi$ and $g$ (see Figure 1).

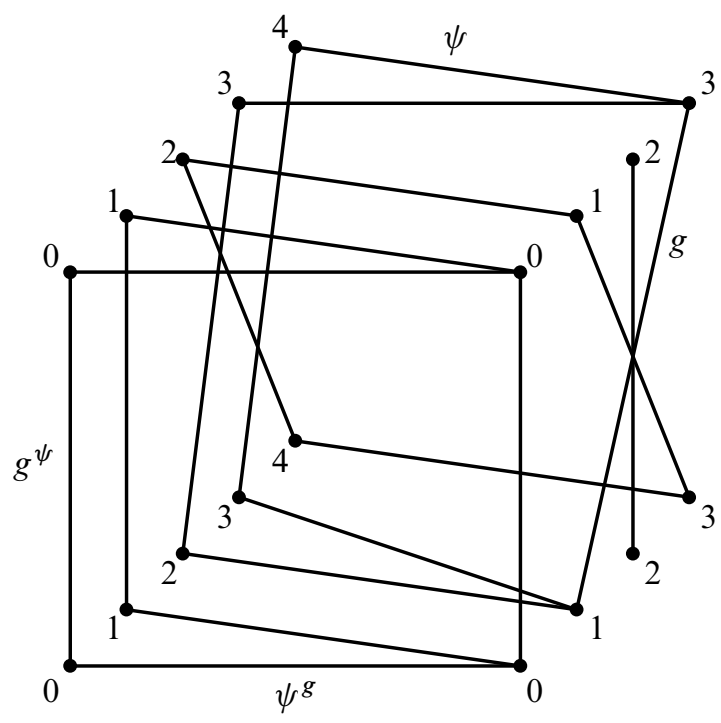

Figure 1: Morphisms of $\Delta S: g \cdot \psi=\left(g^{*}(\psi), \psi^{*}(g)\right)=\left(\psi^{g}, g^{\psi}\right)$

An equivalent characterization of $\Delta S$ comes from Pirashvili, as the category $\mathcal{F}$ (as) of non-commutative sets [22]. The objects are sets $\underline{n} \stackrel{\text { def }}{=}\{1,2, \ldots, n\}$ for $n \geq 0$. By convention, $\underline{0}$ is the empty set. A morphism in $\operatorname{Mor}_{\mathcal{F}(\text { as) }}(\underline{n}, \underline{m})$ consists of a set map $f: \underline{n} \rightarrow \underline{m}$ together with a total ordering on each preimage set $f^{-1}(j)$. There is an obvious inclusion of categories, $\Delta S \hookrightarrow \mathcal{F}$ (as), taking $[n]$ to $\underline{n+1}$, but there is no object of $\Delta S$ that maps to $\underline{0}$. It will be useful to define $\Delta S+\supset \Delta S$ which is isomorphic to $\mathcal{F}($ as) :

Definition $7 \Delta S_{+}$is the category consisting of all objects and morphisms of $\Delta S$, with the additional object $[-1]$, representing the empty set, and a unique morphism $\iota_{n}:[-1] \rightarrow[n]$ for each $n \geq-1$.

Remark 8 Pirashvili's construction is a special case of a more general construction due to May and Thomason [20]. This construction associates to any topological operad $\{\mathcal{C}(n)\}_{n \geq 0}$ a topological category $\widehat{\mathcal{C}}$ together with a functor $\widehat{\mathcal{C}} \rightarrow \mathcal{F}$, where $\mathcal{F}$ is the category of finite sets, such that the inverse image of any function $f: \underline{m} \rightarrow \underline{n}$ is the space $\prod_{i=1}^{n} \mathcal{C}\left(\# f^{-1}(i)\right)$. Composition in $\hat{\mathcal{C}}$ is defined using the composition of the operad. May and Thomason refer to $\hat{\mathcal{C}}$ as the category of operators associated to $\mathcal{C}$. 
They were interested in the case of an $E_{\infty}$ operad, but their construction evidently works for any operad. The category of operators associated to the discrete $A_{\infty}$ operad Ass, which parametrizes monoid structures, is precisely Pirashvili's construction of $\mathcal{F}($ as $)$, that is, $\Delta S_{+}$.

One very useful advantage in enlarging our category to $\Delta S$ to $\Delta S_{+}$is the added structure inherent in $\Delta S_{+}$.

\section{Proposition $9 \Delta S_{+}$is a permutative category.}

Proof Define the monoid product on objects by $[n] \odot[m] \stackrel{\text { def }}{=}[n+m+1]$, (disjoint union of sets), and on morphisms $(\phi, g):[n] \rightarrow\left[n^{\prime}\right],(\psi, h):[m] \rightarrow\left[m^{\prime}\right]$, by $(\phi, g) \odot(\psi, h)=$ $(\eta, k):[n+m+1] \rightarrow\left[n^{\prime}+m^{\prime}+1\right]$, where $(\eta, k)$ is just the morphism $(\phi, g)$ acting on the first $n+1$ points of $[n+m+1]$, and $(\psi, h)$ acting on the remaining points.

The unit object will be $[-1]=\varnothing . \odot$ is clearly associative, and $[-1]$ acts as two-sided identity. Finally, define the transposition transformation $\gamma_{n, m}:[n] \odot[m] \rightarrow[m] \odot[n]$ to be the identity on objects, and on morphisms to be precomposition with the block transposition that switches the first block of size $n+1$ with the second block of size $m+1$.

Remark 10 The fact that $\Delta S_{+}$is permutative shall be exploited to prove that $H S_{*}(A)$ admits homology operations in a forthcoming paper.

Remark 11 It will become convenient to include the object $[-1]=\varnothing$ in $\Delta$ as well. Denote the enlarged category by $\Delta_{+}$.

For the purposes of computation, a morphism $\alpha:[n] \rightarrow[m]$ of $\Delta S$ may be conveniently represented as a tensor product of monomials in the formal non-commuting variables $\left\{x_{0}, x_{1}, \ldots, x_{n}\right\}$. Let $\alpha=(\phi, g)$, with $\phi \in \operatorname{Mor}_{\Delta}([n],[m])$ and $g \in \Sigma_{n+1}^{\mathrm{op}}$. The tensor representation of $\alpha$ will have $m+1$ tensor factors. Each $x_{i}$ will occur exactly once, in the order $x_{g(0)}, x_{g(1)}, \ldots, x_{g(n)}$. The $i$ th tensor factor consists of the product of $\# \phi^{-1}(i-1)$ variables, with the convention that the empty product will be denoted 1 . Thus, the $i$ th tensor factor records the total ordering of $\phi^{-1}(i)$. As an example, the tensor representation of the morphism depicted in Figure 2 is $x_{1} x_{0} \otimes x_{3} x_{4} \otimes 1 \otimes x_{2}$. With this notation, the composition of two morphisms $\alpha=X_{0} \otimes X_{1} \otimes \cdots \otimes X_{m}:[n] \rightarrow[m]$ and $\beta=Y_{1} \otimes Y_{2} \otimes \cdots Y_{n}:[p] \rightarrow[n]$ is given by, $\alpha \beta=Z_{0} \otimes Z_{1} \otimes \cdots \otimes Z_{m}$, where $Z_{i}$ is determined by replacing each variable in the monomials $X_{i}=x_{j_{1}} \ldots x_{j_{s}}$ by the corresponding monomials $Y_{j_{k}}$ in $\beta$. So, $Z_{i}=Y_{j_{1}} \ldots Y_{j_{s}}$. 


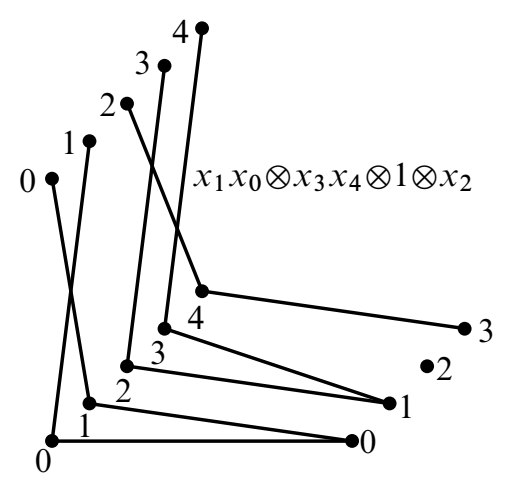

Figure 2: Morphisms of $\Delta S$ in tensor notation

\subsection{Homological algebra of functors}

Recall, for a category $\mathscr{b}$, a $\mathscr{C}$-module is covariant functor $F: \mathscr{b} \rightarrow k$-Mod. Similarly, a $\mathscr{C}^{\mathrm{op}}$-module is a contravariant functor $G: \mathscr{C} \rightarrow k$-Mod. Let $M$ be a $\mathscr{C}^{\mathrm{op}}$-module and $N$ be a $\mathscr{C}$-module. Following Mac Lane [17], define the tensor product of functors as a coend, $M \otimes_{\mathscr{C}} N=\int^{X}(M X) \otimes(N X)$. That is,

$$
M \otimes_{\mathscr{b}} N=\bigoplus_{X \in \mathrm{Obj} \mathscr{C}} M(X) \otimes_{k} N(X) / \approx,
$$

where the equivalence $\approx$ is generated by $y \otimes f_{*}(x) \approx f^{*}(y) \otimes x$ for $f \in \operatorname{Mor}_{\mathscr{C}}(X, Y)$, $x \in N(X)$ and $y \in M(Y)$.

Remark 12 Note, the existing literature based on the work of Connes, Loday and Quillen consistently defines the categorical tensor product in the reverse sense: $N \otimes_{\complement} M$ is the direct sum of copies of $N X \otimes_{k} M X$ modded out by the equivalence $x \otimes f^{*}(y) \approx$ $f_{*}(x) \otimes y$ for all $\mathscr{C}$-morphisms $f: X \rightarrow Y$. In this context, $N$ is covariant, while $M$ is contravariant. I chose to follow the convention of Pirashvili and Richter [23] in writing tensor products as $M \otimes_{\mathscr{C}} N$ so that the equivalence $\xi: \mathscr{C}-\operatorname{Mod} \rightarrow k[\operatorname{Mor} \mathscr{C}]-\operatorname{Mod}$ passes to tensor products in a straightforward way: $\xi\left(M \otimes_{\mathscr{C}} N\right)=\xi(M) \otimes_{k[\operatorname{Mor} \mathscr{C}]} \xi(N)$.

The trivial $\mathscr{C}$-module, resp. $\mathscr{C}^{\text {op }}$-module, denoted by $\underline{k}$ (for either variance), is the functor taking each object to $k$ and each morphism to the identity. As noted in Fiedorowicz-Loday [10], the category of $\mathscr{C}$-modules is abelian and has enough projectives. For any $\mathscr{C}^{\mathrm{op}}$-module $M$, the functor $N \mapsto M \otimes_{\mathscr{C}} N$ is right-exact and so admits derived functors $\operatorname{Tor}_{n}^{\mathscr{C}}(M,-)$ such that $\operatorname{Tor}_{0}^{\mathscr{C}}(M, N)=M \otimes_{\mathscr{C}} N$. 


\subsection{The symmetric bar construction}

Now that we have defined the category $\Delta S$, the next step should be to define an appropriate bar construction. Recall that the cyclic bar construction is a functor $B_{*}^{\text {cyc }} A: \Delta C^{\text {op }} \rightarrow k-$ Mod. One then takes the groups $\operatorname{Tor}_{n}^{\Delta C}\left(B_{*}^{\text {cyc }} A, \underline{k}\right)$ as the definition of $H C_{n}(A)$ for $n \geq 0$. However the results of [10] show that the cyclic bar construction does not extend to a functor $\Delta S^{\text {op }} \rightarrow k-$ Mod. Furthermore, for any functor $F: \Delta S^{\mathrm{op}} \rightarrow k$-Mod, the groups $\operatorname{Tor}_{n}^{\Delta S}(F, \underline{k})$ simply compute the homology of the underlying simplicial module of $F$ (given by restricting $F$ to $\Delta^{\mathrm{op}}$ ). However, Fiedorowicz discovered that there is a natural extension of the cyclic bar construction not to a contravariant functor on $\Delta S$, but to a covariant functor [8].

Definition 13 Let $A$ be an associative, unital algebra over a commutative ground ring $k$. Define a $\Delta S$-module (that is, a functor $\Delta S \rightarrow k-$ Mod), $B_{*}^{\text {sym }} A$ by

$$
\begin{aligned}
& B_{n}^{\mathrm{sym}} A=B_{*}^{\mathrm{sym}} A[n] \stackrel{\text { def }}{=} A^{\otimes(n+1)} \\
& B_{*}^{\mathrm{sym}} A(\alpha):\left(a_{0} \otimes a_{1} \otimes \cdots \otimes a_{n}\right) \mapsto \alpha\left(a_{0}, \ldots, a_{n}\right),
\end{aligned}
$$

where $\alpha:[n] \rightarrow[m]$ is represented in tensor notation, and evaluation at $\left(a_{0}, \ldots, a_{n}\right)$ simply amounts to substituting each $a_{i}$ for $x_{i}$ and multiplying the resulting monomials in $A$. If the pre-image $\alpha^{-1}(i)$ is empty, then the unit of $A$ is inserted. Observe that $B_{*}^{\text {sym }} A$ is natural in $A$.

Note that there are natural inclusions $\Delta \hookrightarrow \Delta C \hookrightarrow \Delta S$. The second inclusion is induced from inclusions of groups $C_{n+1} \hookrightarrow \Sigma_{n+1}$. To be precise, for each $n$, let $\tau_{n}$ be the $(n+1)$-cycle $(0, n, n-1, \ldots, 1) \in \Sigma_{n+1} \cdot \tau_{n}$ generates a subgroup isomorphic to $C_{n+1} . B_{*}^{\text {sym }} A$ may be regarded as a simplicial $k$-module via the chain of functors, $\Delta^{\mathrm{op}} \hookrightarrow \Delta C^{\mathrm{op}} \stackrel{\cong}{\longrightarrow} \Delta C \hookrightarrow \Delta S$. Here, the isomorphism $D: \Delta C^{\mathrm{op}} \rightarrow \Delta C$ is the standard duality (see Loday [16]). Note that the cyclic bar construction can be recovered from the covariant symmetric bar construction by $B_{*}^{\text {cyc }} A=B_{*}^{\text {sym }} A \circ D$.

Definition 14 The symmetric homology of an associative, unital $k$-algebra $A$ is denoted $H S_{*}(A)$, and is defined as

$$
H S_{*}(A) \stackrel{\text { def }}{=} \operatorname{Tor}_{*}^{\Delta S}\left(\underline{k}, B_{*}^{\text {sym }} A\right)
$$

Remark 15 Since $\underline{k} \otimes \Delta S M \cong \operatorname{colim}_{\Delta S} M$, for any $\Delta S$-module $M$, we can alternatively describe symmetric homology as derived functors of the colimit

$$
H S_{n}(A)=\operatorname{colim}_{\Delta S}{ }^{(n)} B_{*}^{\text {sym }} A .
$$


In the language of Gabriel-Zisman [11, Appendix II.3], $H S_{*}(A)=H_{*}\left(\Delta S, B_{*}^{\mathrm{sym}} A\right)$. Moreover, a simplicial module whose homology computes $H_{*}\left(\Delta S, B_{*}^{\text {sym }} A\right)$ is readily available [11, page 153]. In order to describe this simplicial module, we recall that the nerve of a category $\mathscr{C}$ is the simplicial set $N^{\mathscr{C}}$ such that $n$-chains are sequences of $n$ composable morphisms of $\mathscr{C}, 0$-chains are simply objects of $\mathscr{C}$, and face maps are given by deletion of objects along with the composition of the corresponding morphisms. If $F: \mathscr{C} \rightarrow \mathscr{D}$ is a morphism and $\chi \in N \mathscr{b}$ is an $n$-chain,

$$
\chi_{n} \stackrel{f_{n}}{\longleftarrow} \chi_{n-1} \stackrel{f_{n-1}}{\longleftarrow} \cdots \stackrel{f_{2}}{\longleftarrow} \chi_{1} \stackrel{f_{1}}{\longleftarrow} \chi_{0},
$$

then let $F \chi=F \chi_{0}$. The following simplicial module computes $H_{*}\left(\Delta S, B_{*}^{\mathrm{sym}} A\right)$ :

$$
\left\{C_{n}\left(\Delta S, B_{*}^{\mathrm{sym}} A\right)\right\}_{n \geq 0}=\left\{\bigoplus_{\chi \in N_{n} \Delta S} B_{*}^{\mathrm{sym}} A \chi\right\}_{n \geq 0},
$$

with face maps and degeneracies defined by:

$$
\begin{aligned}
& d_{i}(\chi, x)= \begin{cases}\left(d_{0} \chi,\left(f_{1}\right)_{*}(x)\right), & i=0, \\
\left(d_{i} \chi, x\right), & 1 \leq i \leq n\end{cases} \\
& s_{i}(\chi, x)=\left(s_{i} \chi, x\right)
\end{aligned}
$$

In this formula, we have $\chi \in N_{n} \Delta S$ and $x \in B_{*}^{\text {sym }} A \chi$.

$C_{n}\left(\Delta S, B_{*}^{\text {sym }} A\right)$ may be interpreted in terms of the categorical tensor product construction mentioned in Section 1.2, using the under-category functor, $(-\backslash \Delta S): \Delta S^{\mathrm{op}} \rightarrow$ cat, which associates to each object $[p]$ of $\Delta S$, the category $[p] \backslash \Delta S$ of objects under [ $p$ ] (see Quillen [24], Mac Lane [18], etc). The $n$-chains of $N([p] \backslash \Delta S)$ may be identified with $(n+1)$-chains of $N \Delta S$ which have domain $[p]$. Let $k[-]$ be the functor that associates to any simplicial set the corresponding simplicial $k$-module. We may identify

$$
C_{*}\left(\Delta S, B_{*}^{\mathrm{sym}} A\right)=k[N(-\backslash \Delta S)] \otimes_{\Delta S} B_{*}^{\mathrm{sym}} A .
$$

Indeed, observe that every element of $k[N(-\backslash \Delta S)] \otimes_{\Delta S} B_{*}^{\text {sym }} A$ of the form

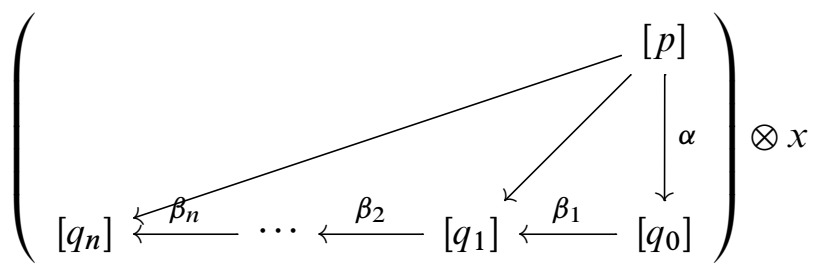


is equivalent to one in which the incoming morphism $\alpha$ becomes an identity:

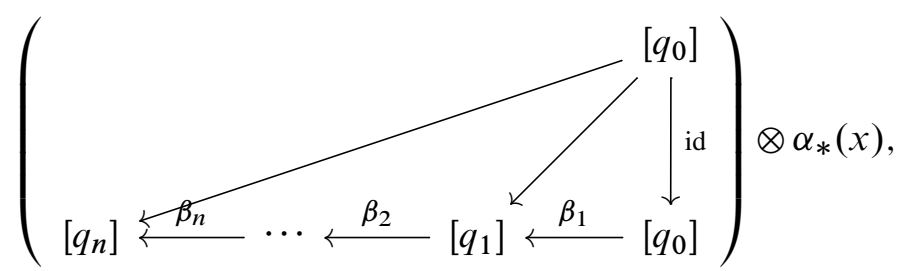

the latter element being identified with

$$
\left(\left[q_{n}\right] \stackrel{\beta_{n}}{\longleftarrow} \cdots \stackrel{\beta_{2}}{\longleftarrow}\left[q_{1}\right] \stackrel{\beta_{1}}{\longleftarrow}\left[q_{0}\right], \alpha_{*}(x)\right) \in C_{n}\left(\Delta S, B_{*}^{\mathrm{sym}} A\right) .
$$

Proposition 16 For an associative, unital $k$-algebra $A$,

$$
H S_{*}(A)=H_{*}\left(k[N(-\backslash \Delta S)] \otimes_{\Delta S} B_{*}^{\mathrm{sym}} A ; k\right) .
$$

Remark 17 By duality of $\Delta C$, it is clear that the related complex $k[N(-\backslash \Delta C)] \otimes_{\Delta C}$ $B_{*}^{\mathrm{sym}} A$ computes $H C_{*}(A)$, where we understand that the functor $B_{*}^{\mathrm{sym}} A$ is restricted to $\Delta C \hookrightarrow \Delta S$.

\subsection{Symmetric homology of the ground ring}

We now have enough tools to compute $H S_{*}(k)$. First, we need to show:

Lemma $18 N(\Delta S)$ is contractible.

Proof Define a functor $\mathscr{F}: \Delta S \rightarrow \Delta S$ on objects by $\mathscr{F}[n]=[0] \odot[n]$, and on morphisms by $\mathscr{F} f=\operatorname{id}_{[0]} \odot f$, using the monoid multiplication $\odot$ defined in Proposition 9 . There is a natural transformation $\mathrm{id}_{\Delta S} \rightarrow \mathscr{F}$ given by the following commutative diagram for each $f:[m] \rightarrow[n]:$

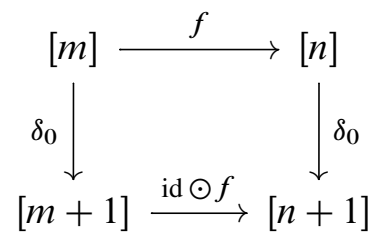

Here, $\delta_{j}^{(k)}:[k-1] \rightarrow[k]$ is the $\Delta$ morphism that misses the point $j \in[k]$.

Consider the constant functor $\Delta S \stackrel{[0]}{\longrightarrow} \Delta S$ that sends all objects to [0] and all morphisms to $\mathrm{id}_{[0]}$. There is a natural transformation $[0] \rightarrow \mathscr{F}$ given by the following commutative 
diagram for each $f:[m] \rightarrow[n]$.

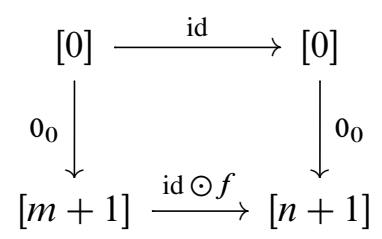

Here, $0_{j}^{(k)}:[0] \rightarrow[k]$ is the morphism that sends the point 0 to $j \in[k]$.

Natural transformations induce homotopy equivalences (see Segal [26] or Quillen [24, Proposition 1.2]), so in particular, the identity map on $N(\Delta S)$ is homotopic to the map that sends $N(\Delta S)$ to the nerve of a trivial category. Thus, $N(\Delta S)$ is contractible.

Corollary 19 The symmetric homology of the ground ring $k$ is isomorphic to $k$, concentrated in degree 0 .

Proof $H S_{*}(k)$ is the homology of the chain complex generated (freely) over $k$ by the chains

$$
\left\{\left[q_{n}\right] \stackrel{\beta_{n}}{\longleftarrow} \cdots \stackrel{\beta_{2}}{\longleftarrow}\left[q_{1}\right] \stackrel{\beta_{1}}{\longleftarrow}\left[q_{0}\right] \otimes(1 \otimes \cdots \otimes 1)\right\},
$$

where $\beta_{i} \in \operatorname{Mor}_{\Delta S}\left(\left[q_{i-1}\right],\left[q_{i}\right]\right)$. Each such chain may be identified with the chain

$$
\left[q_{n}\right] \stackrel{\beta_{n}}{\longleftarrow} \ldots \stackrel{\beta_{2}}{\longleftarrow}\left[q_{1}\right] \stackrel{\beta_{1}}{\longleftarrow}\left[q_{0}\right]
$$

of $N(\Delta S)$, and this defines a chain isomorphism to $N(\Delta S)$. The result now follows from Lemma 18.

\section{Symmetric homology with coefficients and the UCT}

Following the conventions for Hochschild and cyclic homology in Loday [16], when we need to indicate explicitly the ground ring $k$ over which we compute symmetric homology of $A$, we shall use the notation: $H S_{*}(A \mid k)$. On the other hand, for a $k$-module $M, H S_{*}(A ; M)$ will denote the homology of the complex $C_{*}\left(\Delta S, B_{*}^{\mathrm{sym}} A\right) \otimes_{k} M$.

Two easy propositions are collected here for reference.

\section{Proposition 20}

(1) If $M$ is flat over $k$, then $H S_{*}(A ; M) \cong H S_{*}(A) \otimes_{k} M$.

(2) If $B$ is a commutative $k$-algebra, then $H S_{*}\left(A \otimes_{k} B \mid B\right) \cong H S_{*}(A ; B)$. 
Proof Left to the reader.

Theorem 21 (Universal Coefficient Theorem) If $A$ is a flat $k$-algebra, and $B$ is a commutative $k$-algebra, then there is a spectral sequence with

$$
E_{2}^{p, q}=\operatorname{Tor}_{p}^{k}\left(H S_{q}(A \mid k), B\right) \Rightarrow H S_{*}(A \mid B) .
$$

Proof Let $T_{q}: k$-Mod $\rightarrow k$-Mod be the functor $H S_{q}(A ;-)$. Observe, since $A$ is flat, $\left\{T_{q}\right\}$ is a long exact sequence of additive covariant functors (see Dold [6, Definition 1.1] and also McCleary [21, Section 12.1] for details). $T_{q}=0$ for sufficiently small $q$ (indeed, for $q<0$ ) and $T_{q}$ commutes with arbitrary direct sums. Hence, by the Universal Coefficient Theorem [6, Theorem 2.12] (see also [21, Theorem 12.11]), there is a spectral sequence with $E_{2}^{p, q}=\operatorname{Tor}_{p}^{k}\left(T_{q}(k), B\right) \Rightarrow T_{*}(B)$.

As an immediate consequence, we have the following result.

Corollary 22 If $f: \underset{\cong}{A} \rightarrow A^{\prime}$ is a $k$-algebra map between flat algebras inducing an isomorphism $H S_{*}(A) \stackrel{\cong}{\cong} H S_{*}\left(A^{\prime}\right)$ in symmetric homology, then for a commutative $k$ algebra $B$, the chain map $f \otimes \mathrm{id}_{B}$ induces an isomorphism $H S_{*}(A ; B) \stackrel{\cong}{\longrightarrow} H S_{*}\left(A^{\prime} ; B\right)$.

Under stronger hypotheses, the universal coefficient spectral sequence reduces to short exact sequences.

Corollary 23 If $k$ has weak global dimension $\leq 1$, then the spectral sequence of Theorem 21 reduces to short exact sequences,

$$
0 \longrightarrow H S_{n}(A \mid k) \otimes_{k} B \longrightarrow H S_{n}(A ; B) \longrightarrow \operatorname{Tor}_{1}^{k}\left(H S_{n-1}(A \mid k), B\right) \longrightarrow 0 .
$$

Moreover, if $k$ is hereditary and and $A$ is projective over $k$, then these sequences split (unnaturally). (See Itô [13] for precise definitions of weak global dimension and hereditary.)

Proof Assume first that $k$ has weak global dimension $\leq 1$. So $\operatorname{Tor}_{p}^{k}\left(H S_{q}(A \mid k), B\right)=$ 0 for all $p>1$. Following Dold's argument [6, Corollary 2.13], we obtain the required exact sequences. Assume further that $k$ is hereditary and $A$ is projective. Then Rotman [25, Theorem 8.22] gives us the desired splitting.

Remark 24 The proof given above also proves UCT for cyclic homology. A partial result along these lines exists in Loday [16, 2.1.16]. There, he shows $H C_{*}(A \mid k) \otimes_{k}$ $K \cong H C_{*}(A \mid K)$ and $H H_{*}(A \mid k) \otimes_{k} K \cong H H_{*}(A \mid K)$ in the case that $K$ is a localization of $k$, and $A$ is a $K$-module, flat over $k$. I am not aware of a statement of UCT for cyclic or Hochschild homology in its full generality in the literature. 


\section{Integral symmetric homology and a Bockstein spectral se- quence}

We shall obtain a converse to Corollary 22 in the case $k=\mathbb{Z}$.

Theorem 25 Let $f: A \rightarrow A^{\prime}$ be an algebra map between torsion-free $\mathbb{Z}$-algebras. Suppose that for $B=\mathbb{Q}$ or $B=\mathbb{Z} / p \mathbb{Z}$ (for any prime $p$ ) the map

$$
f \otimes \operatorname{id}_{B}: C_{*}\left(\Delta S, B_{*}^{\mathrm{sym}} A\right) \otimes_{k} B \longrightarrow C_{*}\left(\Delta S, B_{*}^{\mathrm{sym}} A^{\prime}\right) \otimes_{k} B
$$

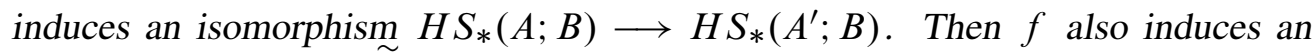
isomorphism $H S_{*}(A) \stackrel{\cong}{\cong} H S_{*}\left(A^{\prime}\right)$.

First, note that if $A$ is flat over $k$, one can construct the Bockstein homomorphisms $\beta_{n}: H S_{n}(A ; Z) \rightarrow H S_{n-1}(A ; X)$ associated to a short exact sequence of $k$-modules, $0 \rightarrow X \rightarrow Y \rightarrow Z \rightarrow 0$. These Bockstein maps are natural in $A$, since $H S_{*}(A ;-)$ is a long exact sequence of functors (see Section 2). Moreover if the induced map $f_{*}: H S_{*}(A ; W) \rightarrow H S_{*}\left(A^{\prime} ; W\right)$ is an isomorphism for any two of $W=X, W=Y$ and $W=Z$, then it is an isomorphism for the third - an easy exercise for the reader. We shall now proceed with the proof of Theorem 25 . All tensor products will be over $\mathbb{Z}$ in what follows.

Proof Let $A$ and $A^{\prime}$ be torsion-free $\mathbb{Z}$-modules. Let $f: A \rightarrow A^{\prime}$ be an algebra map inducing isomorphism in symmetric homology with coefficients in $\mathbb{Q}$ and also in $\mathbb{Z} / p \mathbb{Z}$ for any prime $p$. For $m \geq 2$, there is a short exact sequence,

$$
0 \longrightarrow \mathbb{Z} / p^{m-1} \mathbb{Z} \stackrel{p}{\longrightarrow} \mathbb{Z} / p^{m} \mathbb{Z} \longrightarrow \mathbb{Z} / p \mathbb{Z} \longrightarrow 0 .
$$

A straightforward induction argument shows that the maps induced by $f$ are isomorphisms,

$$
f_{*}: H S_{*}\left(A ; \mathbb{Z} / p^{m} \mathbb{Z}\right) \stackrel{\cong}{\longrightarrow} H S_{*}\left(A^{\prime} ; \mathbb{Z} / p^{m} \mathbb{Z}\right)
$$

Denote $\mathbb{Z} / p^{\infty} \mathbb{Z}=\lim \mathbb{Z} / p^{m} \mathbb{Z}$. Note, this is a direct limit in the sense that it is a colimit over a directed system. The direct limit functor is exact (see Stenström [28, Proposition 5.3]), so the maps $H S_{n}\left(A ; \mathbb{Z} / p^{\infty} \mathbb{Z}\right) \rightarrow H S_{n}\left(A^{\prime} ; \mathbb{Z} / p^{\infty} \mathbb{Z}\right)$ induced by $f$ are isomorphisms, given by the following chain of isomorphisms:

$$
\begin{aligned}
H S_{n}\left(A ; \mathbb{Z} / p^{\infty} \mathbb{Z}\right) & \cong \lim _{*} H_{*}\left(k\left[C_{*}\left(\Delta S, B_{*}^{\mathrm{sym}} A\right)\right] \otimes \mathbb{Z} / p^{m} \mathbb{Z}\right) \\
\stackrel{f_{*}}{\longrightarrow} & \stackrel{\lim }{\longrightarrow} H_{*}\left(k\left[C_{*}\left(\Delta S, B_{*}^{\mathrm{sym}} A^{\prime}\right)\right] \otimes \mathbb{Z} / p^{m} \mathbb{Z}\right) \cong H S_{*}\left(A^{\prime} ; \mathbb{Z} / p^{\infty} \mathbb{Z}\right)
\end{aligned}
$$


(Note that $f_{*}$ here stands for $\underset{\lim }{\longrightarrow} H_{n}\left(k\left[C_{*}\left(\Delta S, B_{*}^{\text {sym }} f\right)\right] \otimes\right.$ id $)$.)

Finally, consider the short exact sequence of abelian groups,

$$
0 \longrightarrow \mathbb{Z} \longrightarrow \mathbb{Q} \longrightarrow \underset{p \text { prime }}{\bigoplus} \mathbb{Z} / p^{\infty} \mathbb{Z} \longrightarrow 0
$$

The isomorphism $f_{*}: H S_{*}\left(A ; \mathbb{Z} / p^{\infty} \mathbb{Z}\right) \rightarrow H S_{*}\left(A^{\prime} ; \mathbb{Z} / p^{\infty} \mathbb{Z}\right)$ passes to direct sums, giving isomorphisms

$$
f_{*}: H S_{n}\left(A ; \bigoplus_{p} \mathbb{Z} / p^{\infty} \mathbb{Z}\right) \stackrel{\cong}{\longrightarrow} H S_{n}\left(A^{\prime} ; \bigoplus_{p} \mathbb{Z} / p^{\infty} \mathbb{Z}\right)
$$

for each $n$. Since $H S_{*}(A ; \mathbb{Q}) \rightarrow H S_{*}\left(A^{\prime} ; \mathbb{Q}\right)$ is an isomorphism, we have the required isomorphism in symmetric homology, $f_{*}: H S_{n}(A \mid \mathbb{Z}) \stackrel{\cong}{\longrightarrow} H S_{n}\left(A^{\prime} \mid \mathbb{Z}\right)$.

Remark 26 Theorem 25 may be useful for determining integral symmetric homology, since rational computations are generally simpler, and computations mod $p$ may be made easier due to the presence of additional structure, such as homology operations (see the author's forthcoming paper).

Finally, we state a result along the lines of McCleary [21, Theorem 10.3]. Denote the torsion submodule of a graded module $H_{*}$ by $\tau\left(H_{*}\right)$.

Theorem 27 (Bockstein spectral sequence) Suppose $A$ is free of finite rank over $\mathbb{Z}$. Then there is a singly-graded spectral sequence with

$$
E_{*}^{1}=H S_{*}(A ; \mathbb{Z} / p \mathbb{Z}) \Rightarrow H S_{*}(A) / \tau\left(H S_{*}(A)\right) \otimes \mathbb{Z} / p \mathbb{Z},
$$

with differential map $d^{1}=\beta$, the standard Bockstein map associated to

$$
0 \rightarrow \mathbb{Z} / p \mathbb{Z} \rightarrow \mathbb{Z} / p^{2} \mathbb{Z} \rightarrow \mathbb{Z} / p \mathbb{Z} \rightarrow 0 .
$$

Moreover, the convergence is strong.

Proof The proof McCleary gives [21, page 459] carries over to our case intact. All that is required for this proof is that each $H S_{n}(A)$ be a finitely-generated abelian group. The hypothesis that $A$ is finitely-generated, coupled with the result of Corollary 78 of Section 9.4, guarantees this.

\section{Symmetric homology using $\Delta S_{+}$}

In this section, we shall show that replacing $\Delta S$ by $\Delta S_{+}$in an appropriate way does not affect the computation of $H S_{*}$. We shall define a functor $B_{*}^{\text {sym }_{+}} A: \Delta S_{+} \rightarrow k-M o d$

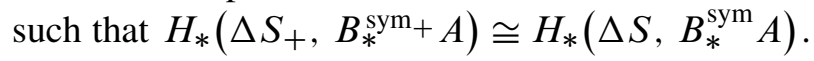




\subsection{Extension of the bar construction to $\Delta S_{+}$}

Recall for a functor $\mathscr{F}: \mathscr{D} \rightarrow \mathscr{C}$ of small categories, the induced functor from the category of $\mathscr{C}$-modules to $\mathscr{D}$-modules given by $M \mapsto M \circ \mathscr{F}$ admits a left adjoint functor, $\mathscr{F}^{*}$ (see Kan [14] or Gabriel-Zisman [11, Appendix II.3]). In particular, the inclusion $i: \Delta S \hookrightarrow \Delta S_{+}$induces a functor $i^{*}: \Delta S-\operatorname{Mod} \longrightarrow \Delta S_{+}-\operatorname{Mod}$. It is instructive to analyze the functor $i^{*}\left(B_{*}^{\mathrm{sym}} A\right): \Delta S_{+} \rightarrow k-\operatorname{Mod}$. Note, $i^{*}\left(B_{*}^{\mathrm{sym}} A\right)$ is just the left Kan extension of $B_{*}^{\text {sym }} A$ along $i$. By definition, $i^{*}\left(B_{*}^{\text {sym }} A\right)$ is the functor, $[n] \mapsto \operatorname{colim}_{(i /[n])} B_{*}^{\text {sym }} A \circ \pi_{1}$, where $\pi_{1}:(i /[n]) \rightarrow \Delta S$ is the projection $(i[m] \stackrel{\phi}{\rightarrow}[n]) \mapsto[m]$. Note, for $n=-1, \operatorname{colim}_{(i /[-1])} B_{*}^{\text {sym }} A \circ \pi_{1} \cong 0$, the initial object of $k$-Mod, since the category $(i /[-1])$ is empty. For $n \geq 0$,

(2) $\underset{(i /[n])}{\operatorname{colim}} B_{*}^{\mathrm{sym}} \circ \pi_{1}=\left(\begin{array}{c}\bigoplus \\ ([n] \stackrel{\phi}{\leftarrow}[m]) \in \operatorname{Mor} \Delta S \\ B_{m}^{\mathrm{sym}} A\end{array}\right) /(\phi \psi, x) \approx\left(\phi, \psi_{*}(x)\right) \cong B_{n}^{\mathrm{sym}} A$

The last isomorphism results from the "identity trick" (see, for example, Section 1.3), namely $(\phi, x) \approx\left(\mathrm{id}, \phi_{*}(x)\right)$. For any morphism $\beta \in$ Mor $\Delta S$, the morphism induced by $i^{*}\left(B_{*}^{\text {sym }} A\right)$ is simply $\beta$ itself. On the other hand, the morhpism $\iota_{n}:[-1] \rightarrow[n]$ of $\Delta S_{+}$becomes the zero map $0 \rightarrow A^{\otimes(n+1)}$ under $i^{*}\left(B_{*}^{\mathrm{sym}} A\right)$.

Definition 28 Given an associative, unital algebra $A$ over a commutative ground ring $k$, we let $B_{*}^{\text {sym }}+A$ be the functor $i^{*}\left(B_{*}^{\text {sym }} A\right): \Delta S_{+} \rightarrow k-$ Mod defined above.

Lemma $29 H_{*}\left(\Delta S_{+}, B_{*}^{\mathrm{sym}_{+}^{0}} A\right) \cong H_{*}\left(\Delta S, B_{*}^{\mathrm{sym}} A\right)$.

Proof By Gabriel-Zisman [11, Theorem 3.6], there is a spectral sequence with

$$
E_{p, q}^{2}=\underset{\Delta S_{+}}{\operatorname{colim}}\left(L_{q} i^{*}\right)\left(B_{*}^{\text {sym }} A\right) \Rightarrow \underset{\Delta S}{\operatorname{colim}}(p+q) B_{*}^{\text {sym }} A,
$$

where $i$ is the inclusion $\Delta S \hookrightarrow \Delta S_{+}$as above and $L_{q} i^{*}$ is the $q$ th left-derived functor of the right-exact functor $i^{*}$. The target $\operatorname{colim}_{\Delta S}{ }^{(p+q)} B_{*}^{\text {sym }} A$ is the same as $H S_{p+q}(A)$. I claim that $i^{*}$ is in fact left-exact.

Let $0 \rightarrow M \rightarrow N \rightarrow P \rightarrow 0$ be an exact sequence of $\Delta S$-modules. By the same argument displayed in (2), we have

$$
\underset{(i /[n])}{\operatorname{colim}} M \circ \pi_{1} \cong \begin{cases}0, & \text { if } n=-1 \\ M_{n}, & \text { if } n \geq 0\end{cases}
$$

Similar results follow for $N$ and $P$. Thus, the sequence $0 \rightarrow i^{*} M \rightarrow i^{*} N \rightarrow i^{*} P \rightarrow 0$ is exact, since each sequence of $k$-modules, $0 \rightarrow M_{n} \rightarrow N_{n} \rightarrow P_{n} \rightarrow 0$ is exact, and 
certainly the sequence of 0 modules corresponding to $n=-1$ is exact. Now, since $i^{*}$ is shown to be an exact functor, its left-derived functors $L_{q} i^{*}$ must be trivial for $q \geq 1$. The spectral sequence (3) is trivial in columns $q \geq 1$, and thus strongly converges

$$
\underset{\Delta S_{+}}{\operatorname{colim}}(p) i^{*} B_{*}^{\mathrm{sym}} A=H_{p}\left(\Delta S_{+}, B_{*}^{\mathrm{sym}_{+}^{0}} A\right) \Rightarrow H S_{p}(A) .
$$

\subsection{A more useful extension}

Unfortunately, the $\Delta S_{+}-$module $B_{*}^{\text {sym }}+A$ does not seem to reflect the useful properties of $\Delta S_{+}$. In particular, the module corresponding to the object [-1] is trivial in $B_{*}^{\text {sym }}+A$. Define a related functor which is non-trivial at the object $[-1]$ :

Definition 30 For an associative, unital algebra, $A$, over a commutative ground ring $k$, define a functor $B_{*}^{\mathrm{sym}_{+}} A: \Delta S_{+} \rightarrow k-$ Mod by

$$
\left\{\begin{array}{l}
B_{n}^{\mathrm{sym}_{+}} A=A^{\otimes(n+1)}, \text { for } n \geq 0 \\
B_{-1}^{\mathrm{sym}_{+}} A=k,
\end{array}\right.
$$

Morphisms are mapped in the same way as for $B_{*}^{\mathrm{sym}_{+}^{0}} A$ with the exception that $\iota_{n}$ gets mapped to the inclusion $k \hookrightarrow A^{\otimes(n+1)}$ as algebras.

One very useful consequence of defining symmetric in terms of the homology groups $H_{*}(\mathscr{C}, F)$ for an appropriate category $\mathscr{C}$ and functor $F$, is that the functor $H_{*}(\mathscr{C},-)$, takes short exact sequences of $\mathscr{C}$-modules to long exact sequences in homology (see Gabriel-Zisman [11]). We use this fact to prove:

Theorem 31 For an associative, unital $k$-algebra $A, H S_{*}(A)=H_{*}\left(\Delta S_{+}, B_{*}^{\mathrm{sym}_{+}} A\right)$.

Proof Consider the following sequence of $\Delta S_{+}$-modules:

$$
0 \rightarrow B_{*}^{\mathrm{sym}_{+}^{0}} A \rightarrow B_{*}^{\mathrm{sym}_{+}} A \rightarrow Q \rightarrow 0
$$

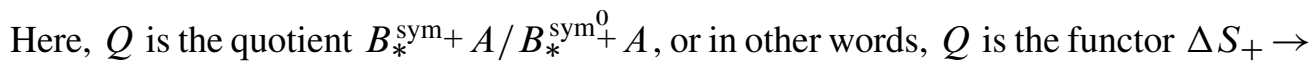
$k$-Mod taking $[-1] \mapsto k$ and $[n] \mapsto 0$ for all $n \geq 0$, which has no dependence on the choice of algebra $A$. Indeed, with $A=k$, we have the exact sequence:

$$
0 \rightarrow B_{*}^{\mathrm{sym}_{+}^{0}} k \rightarrow B_{*}^{\mathrm{sym}_{+}} k \rightarrow Q \rightarrow 0
$$

The corresponding long exact sequence in homology is rather easy to analyze. Using Lemma 29,

$$
\begin{aligned}
H_{p}\left(\Delta S_{+}, B_{*}^{\mathrm{sym}_{+}^{0}} k\right)=H S_{p}(k) & = \begin{cases}k, & p=0 \\
0, & p>0\end{cases} \\
& \cong H_{*}\left(N\left(\Delta S_{+}\right)\right) \cong H_{*}\left(\Delta S_{+}, B_{*}^{\mathrm{sym}_{+}}\right)
\end{aligned}
$$


The last isomorphism is obtained in the same way as in Corollary 19. Thus it follows that $H_{*}\left(\Delta S_{+}, Q\right)=0$. The 5-lemma applied to the long exact sequence corresponding to diagram (4) then proves $H_{n}\left(\Delta S_{+}, B_{*}^{\text {sym }}+A\right) \cong H_{n}\left(\Delta S_{+}, B_{*}^{\text {sym }}+A\right)$ in every degree. Lemma 29 provides the link to symmetric homology.

\section{Tensor algebra decomposition and Fiedorowicz's theorems}

For a general $k$-algebra $A$, finding $H S_{*}(A)$ using the standard resolution is often too difficult. The multiplicative structure of $A$ is hard to control. On the other hand, tensor algebras may be easier to deal with since their multiplicative structure is so clean. Indeed, tensor algebra arguments are also key in the proof of Fiedorowicz's Theorem about the symmetric homology of group algebras.

\subsection{Resolution of algebras using the 2-sided bar construction}

Let $T: k$-Mod $\rightarrow k$-Alg be the functor sending a $k$-module to the tensor algebra generated by that $k$-module. In other words, $T M=\bigoplus_{n \geq 0} M^{\otimes n}$. There is an algebra homomorphism $\theta_{A}: T A \rightarrow A$, defined by multiplying tensor factors, $\theta_{A}\left(a_{0} \otimes a_{1} \otimes \cdots \otimes\right.$ $\left.a_{k}\right)=a_{0} a_{1} \cdots a_{k}$. By abuse of notation, let $T$ also stand for the functor $k-$ Mod $\rightarrow k-$ Mod obtained by composing with the forgetful functor $k-\mathbf{A l g} \rightarrow k-$ Mod. $T$ becomes a monad with multiplication $\theta_{T A}: T^{2} \rightarrow T$ and unit transformation $h:$ id $\rightarrow T$, defined by sending the module $M$ identically onto the summand corresponding to $n=1$. The homomorphism $\theta_{A}$ then expresses $A$ as a $T$-algebra.

Observe that May's 2-sided bar construction $B_{*}(T, T, A)$ (see May [19, Chapter 9]) gives a resolution of $A$ by tensor algebras:

$$
0 \longleftarrow A \stackrel{\theta_{A}}{\longleftarrow} T A \stackrel{\theta_{1}}{\longleftarrow} T^{2} A \stackrel{\theta_{2}}{\longleftarrow} \ldots
$$

The boundary maps $\theta_{n}$ are defined in the standard way: $\theta_{n} \stackrel{\text { def }}{=} \sum_{i=0}^{n}(-1)^{i} T^{n-i} \theta_{T^{i} A}$. If we denote by $A_{*}$ the simplicial algebra consisting of $A$ all degrees and whose faces and degeneracies are all $\mathrm{id}_{A}$, then there is a strong deformation retract $B_{*}(T, T, A) \rightarrow$ $A_{*}$ [19].

\subsection{Monoid algebras}

In the case that $A=k[M]$ for a monoid, $M$, we find a remarkable interpretation of the complex $C_{*}\left(\Delta S_{+}, B_{*}^{\mathrm{sym}_{+}} T A\right)$ as an $E_{\infty}$-algebra. First, define a set-valued variant of the symmetric bar construction: 
Definition 32 Let $M$ be a monoid. Define a functor $B_{*}^{\text {sym }} M: \Delta S \rightarrow$ Sets by:

$$
\begin{array}{ll}
B_{n}^{\text {sym }} M=B_{*}^{\text {sym }} M[n] \stackrel{\text { def }}{=} M^{n+1}, & \text { (set product) } \\
B_{*}^{\text {sym }} M(\alpha):\left(m_{0}, \ldots, m_{n}\right) \mapsto \alpha\left(m_{0}, \ldots, m_{n}\right), & \text { for } \alpha \in \text { Mor } \Delta S .
\end{array}
$$

where $\alpha:[n] \rightarrow[k]$ is represented in tensor notation, and evaluation at $\left(m_{0}, \ldots, m_{n}\right)$ is as in Definition 13.

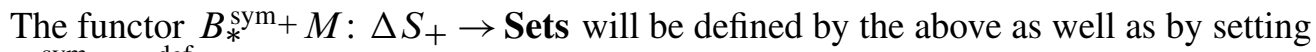
$B_{-1}^{\text {sym }_{+}} M \stackrel{\text { def }}{=} \varnothing$, and $B_{*}^{\text {sym }}+M \iota_{n}$ is the inclusion $\varnothing \hookrightarrow M^{n+1}$.

Regarding $N\left(-\backslash \Delta S_{+}\right)$as a contravariant functor to sets, define $N\left(-\backslash \Delta S_{+}\right) \times \Delta S_{+}$ $B_{*}^{\text {sym }_{+}} M$ as a coend construction similar to that in Section 1.2. It is immediate that this construction is a simplicial set whose homology computes $H S_{*}(k[M])$.

Now, in the context of monoids, the James construction is the appropriate analog of the the tensor algebra construction of Section 5.1. If $M$ is a free monoid on a generating set $X$, that is, $M=J X_{+}$, then $k[M]=k\left[J X_{+}\right]=T(k[X])$ is the free tensor algebra over $k$ on the set $X$ with disjoint basepoint. In this case, we have the following:

\section{Lemma 33}

$$
\left.H S_{*}\left(k\left[J X_{+}\right]\right)\right) \cong H_{*}\left(\coprod_{n \geq-1} \tilde{X}_{n} ; k\right)
$$

where

$$
\tilde{X}_{n}= \begin{cases}N\left(\Delta S_{+}\right), & n=-1 \\ N\left([n] \backslash \Delta S_{+}\right) \times_{\Sigma_{n+1}^{\mathrm{op}}} X^{n+1}, & n \geq 0\end{cases}
$$

Proof Using the simplicial set $N\left(-\backslash \Delta S_{+}\right) \times_{\Delta S_{+}} B_{*}^{\mathrm{sym}_{+}} J X_{+}$, a typical generator has the form,

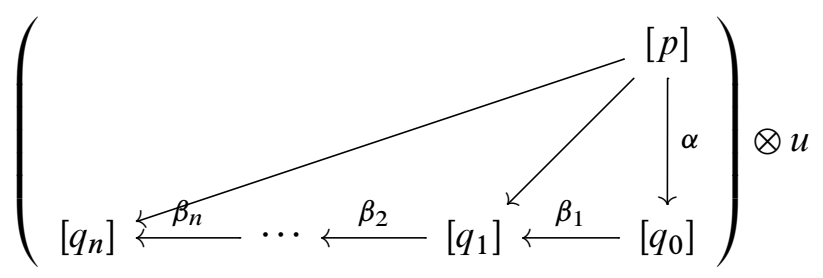

where

$$
u=\left(\bigotimes_{x \in X_{0}} x\right) \otimes\left(\bigotimes_{x \in X_{1}} x\right) \otimes \cdots \otimes\left(\bigotimes_{x \in X_{p}} x\right)
$$


and $X_{0}, X_{1}, \ldots, X_{p}$ are finite ordered lists of elements of $X$. The idea is to "expand" the element $u$ into a tensor of individual elements of $X$, and at the same time to get rid of the units that may be present. Indeed, each $X_{j}$ may be thought of as an element of the set product $X^{m_{j}}$ for some $m_{j}$. If $X_{j}=\varnothing$, then set $m_{j}=0$. We use the convention that an empty tensor product is equal to $1_{k}$ (think of this as the disjoint basepoint appended to $X$ ), and say that the corresponding tensor factor is trivial. Now let $m=\left(\sum m_{j}\right)-1$. Let $\pi: X^{m_{0}} \times X^{m_{1}} \times \cdots \times X^{m_{p}} \longrightarrow X^{m+1}$ be the evident isomorphism. Let $X_{m}=\pi\left(X_{0}, X_{1}, \ldots, X_{p}\right)$.

Case 1 If $u$ is non-trivial (that is, $X_{m} \neq \varnothing$ ), then construct the element

$$
u^{\prime}=\bigotimes_{x \in X_{m}} x
$$

Next, construct a $\Delta$-morphism $\zeta_{u}:[m] \rightarrow[p]$ as follows: For each $j, \zeta_{u}$ maps the points

$$
\sum_{i=0}^{j-1} m_{i},\left(\sum_{i=0}^{j-1} m_{i}\right)+1, \ldots,\left(\sum_{i=0}^{j} m_{i}\right)-1 \mapsto j
$$

Observe, $\left(\zeta_{u}\right)_{*}\left(u^{\prime}\right)=u$. Under $\Delta S_{+}$-equivalence, expression (7) is equivalent to

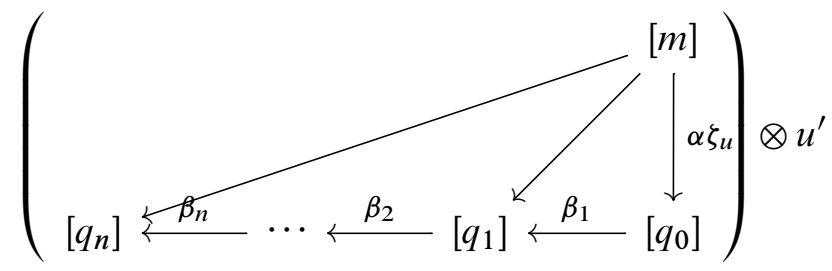

The choice of $u^{\prime}$ and $\zeta_{u}$ is well-defined with respect to the $\Delta S_{+- \text {-equivalence up to }}$ isomorphism of $[m]$ (an element of $\Sigma_{m+1}^{\mathrm{op}}$ ). This shows that any such non-trivial chain may be written uniquely as an element of $N\left([m] \backslash \Delta S_{+}\right) \times_{k} \Sigma_{m+1}^{\text {op }} X^{m+1}$. An example should clarify this process. If $u=\left(x_{i_{0}} \otimes x_{i_{1}}\right) \otimes 1 \otimes\left(x_{i_{2}} \otimes x_{i_{3}}\right) \in(T(k[X]))^{\otimes 3}$, then $u^{\prime}=x_{i_{0}} \otimes x_{i_{1}} \otimes x_{i_{2}} \otimes x_{i_{3}}$ and $\zeta_{u}=x_{0} x_{1} \otimes 1 \otimes x_{2}$ (written in tensor notation - these $x_{i}$ 's are formal variables, not elements of $X$ ). Clearly, $\zeta_{u}\left(u^{\prime}\right)=u$.

Case 2 If $u$ is trivial, then $u=1_{k}^{\otimes(p+1)}$, and we have

$$
\left(\left[q_{n}\right] \longleftarrow \cdots \longleftarrow\left[q_{0}\right] \stackrel{\alpha}{\longleftarrow}[p]\right) \otimes u \approx\left(\left[q_{n}\right] \longleftarrow \cdots \longleftarrow\left[q_{0}\right] \stackrel{l q_{0}}{\longleftarrow}[-1]\right) \otimes 1_{k} .
$$

This element can be identified uniquely with the chain $\left[q_{n}\right] \longleftarrow \cdots \longleftarrow\left[q_{0}\right] \in N\left(\Delta S_{+}\right)$. Thus, the isomorphism (6) is proven. Note that the total number of non-trivial tensor factors is preserved under $\Delta S$ morphisms. This shows that the differential respects the direct sum decomposition. 
Remark 34 This proof works the same using $\Delta S$ rather than $\Delta S_{+}$, by replacing the argument of Case 2 with the "identity trick",

$$
\left(\left[q_{n}\right] \longleftarrow \cdots \longleftarrow\left[q_{0}\right] \stackrel{\alpha}{\longleftarrow}[p]\right) \otimes u \approx\left(\left[q_{n}\right] \longleftarrow \cdots \longleftarrow\left[q_{0}\right] \stackrel{\mathrm{id}}{\longleftarrow}\left[q_{0}\right]\right) \otimes 1_{k}^{\otimes\left(q_{0}+1\right)} .
$$

However the extra structure of $\Delta S_{+}$will be necessary to prove the $E_{\infty}$-structure needed for the results in the next section.

Breaking normal convention, let $X^{0}$ stand for the one-point set, $\{*\}$, (or $\left\{1_{k}\right\}$, which signifies the purpose of this set). Then the content of Lemma 33 can be more concisely,

$$
H S_{*}\left(k\left[J X_{+}\right]\right) \cong \bigoplus_{n \geq-1} H_{*}\left(N\left([n] \backslash \Delta S_{+}\right) \times_{\Sigma_{n+1}^{\mathrm{op}}} X^{n+1} ; k\right)
$$

Remark 35 It will be useful to work with a resolution of the monoid $M$ by free monoids. We obtain an equivalence, $B_{*}(J, J, M) \rightarrow M_{*}$, where $M_{*}$ is the simplicial monoid consisting of $M$ in all degrees and whose faces and degeneracies are all $\mathrm{id}_{M}$.

The next lemma will provide an essential link in the computation of $H S_{*}(k[M])$.

Lemma 36 Let $\mathcal{N} \stackrel{\text { def }}{=} N\left(-\backslash \Delta S_{+}\right) \times_{\Delta S_{+}} B_{*}^{\text {sym }_{+}} J$. There is an equivalence of functors $\Theta: \mathcal{N} \stackrel{\simeq}{\longrightarrow} C_{\infty}$, where $C_{\infty}$ is the monad associated to the $E_{\infty}$-operad, $\mathcal{C}_{\infty}$. Moreover, $\Theta$ induces an equivalence

$$
\Theta_{*}: B_{*}(\mathcal{N}, J, M) \rightarrow B_{*}\left(C_{\infty}, J, M\right)
$$

Remark $37 \mathcal{N}$ itself turns out not to be a full-fledged operad, since it fails the rightunit condition. However, in order to prove Fiedorowicz's Theorems below, all that we require is an equivalence of functors which preserves the simplicial maps of the bar construction.

Proof By definition,

$$
\mathcal{N} X=\coprod_{n \geq 0} N\left([n-1] \backslash \Delta S_{+}\right) \times{ }_{\Sigma_{n}^{\mathrm{op}}} X^{n},
$$

which has been re-indexed to begin at $n=0$. Let $\mathscr{D}$ denote the operad with $\mathscr{D}(n)=E \Sigma_{n}$. Since $\mathscr{D}$ is an $E_{\infty}$-operad, and the monads associated to different $E_{\infty}$-operads are equivalent, it suffices to show the equivalence, $\mathcal{N} X \simeq \mathscr{D} X$.

Note, the 0 -chains (objects) of $N\left([n-1] \backslash \Delta S_{+}\right)$are pairs $(\phi, g) \in \operatorname{Mor}_{\Delta_{+}}([n-$ $1],[m]) \times \Sigma_{n}^{\text {op }}$, so the $\Sigma_{n}$-action is free. Since $N\left([n-1] \backslash \Delta S_{+}\right)$is a contractible 
simplicial set, there is an equivalence induced on 0 -chains by the map sending $(\phi, g) \mapsto$ $g$ :

$$
B\left([n-1] \backslash \Delta S_{+}\right) \stackrel{\simeq}{\rightarrow} E \Sigma_{n}^{\mathrm{op}},
$$

where $B \mathscr{C}$ is the classifying space of a category $\mathscr{C}$. The map $g \rightarrow g^{-1}$ provides an equivalence $E \Sigma_{n}^{\mathrm{op}} \simeq E \Sigma_{n}$.

These equivalences induce

$$
\coprod_{n \geq 0} N\left([n-1] \backslash \Delta S_{+}\right) \times \Sigma_{n}^{\mathrm{op}} X^{n} \simeq \coprod_{n \geq 0} E_{*} \Sigma_{n}^{\mathrm{op}} \times \Sigma_{n}^{\mathrm{op}} X^{n} \simeq \coprod_{n \geq 0} E_{*} \Sigma_{n} \times_{\Sigma_{n}} X^{n} .
$$

Equation (11) gives the equivalence of functors, $\mathcal{N} \rightarrow C_{\infty}$. For the second part of the lemma, we need only consider the 0 -faces of each bar construction in (10), since the other face maps and all of the degeneracies only depend on the monad $J$ and the monoid $M$. The 0 -faces correspond to the transformations $\mathcal{N} J \rightarrow \mathcal{N}$ on the one hand, and $\mathscr{D} J \rightarrow \mathscr{D}$ on the other. Since the map $\mathcal{N} X \rightarrow \mathscr{D} X$ is natural in $X$, the diagram below commutes:

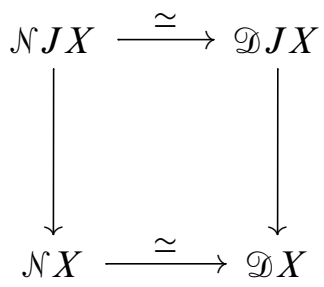

Thus, the equivalence passes to the bar construction with no problem.

\subsection{Fiedorowicz's theorems}

The results of this section have been known for some time now, but not previously published. Fiedorowicz studied the symmetric homology of monoid algebras and group algebras [8]. All proofs of this section are either based on or inspired by the corresponding proofs given in that preprint. The following theorem is the first major result, which serves as a stepping stone to the group-algebra case.

Theorem $38 H S_{*}(k[M]) \cong H_{*}\left(B\left(C_{\infty}, C_{1}, M\right) ; k\right)$, where $C_{1}$ is the little 1-cubes monad and $C_{\infty}$ is the little $\infty$-cubes monad.

Proof The arguments become clearer if we use homotopy colimits throughout. The link is provided by the following fact of homological algebra:

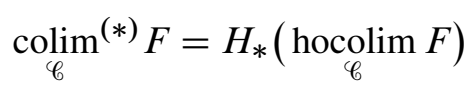


In our case, we are interested in $\mathscr{C}=\Delta S_{+}$and $F=B_{*}^{\mathrm{sym}_{+}} M$. By Section 5.1, we can replace $M$ by its resolution $B_{*}(J, J, M)$. So there is an equivalence,

$$
\underset{\Delta S_{+}}{\operatorname{hocolim}} B_{*}^{\mathrm{sym}_{+}} M \simeq \underset{\Delta S_{+}}{\operatorname{hocolim}} B_{*}^{\mathrm{sym}_{+}} B_{*}(J, J, M)
$$

Now using May [19, Lemma 9.7],

$$
\underset{\Delta S_{+}}{\operatorname{hocolim}} B_{*}^{\mathrm{sym}_{+}} B_{*}(J, J, M) \cong B_{*}\left(\underset{\Delta S_{+}}{\operatorname{hocolim}} B_{*}^{\mathrm{sym}_{+}} J, J, M\right)=B_{*}(\mathcal{N}, J, M)
$$

By Lemma 36 the latter is equivalent to $B_{*}\left(C_{\infty}, J, M\right)$. The proof is completed by using the equivalence $J \simeq C_{1}$.

As we turn our attention to group algebras, the following lemma will be necessary.

Lemma 39 For any topological monoid, $M$, there is a natural equivalence,

$$
B\left(\Omega^{\infty} S^{\infty}, C_{1}, M\right) \simeq \Omega \Omega^{\infty} S^{\infty}(B M)
$$

Proof Consider the bar construction $B_{*}\left(S, C_{1}, M\right)$, where $S$ is suspension. May [19, Lemma 9.7] gives the isomorphism

$$
B_{*}\left(\Omega^{\infty} S^{\infty} S, C_{1}, M\right) \cong \Omega^{\infty} S^{\infty} B_{*}\left(S, C_{1}, M\right) .
$$

Looping on both sides and taking geometric realization, we get

$$
B\left(\Omega \Omega^{\infty} S^{\infty} S, C_{1}, M\right) \cong \Omega \Omega^{\infty} S^{\infty} B\left(S, C_{1}, M\right) .
$$

Now $B\left(\Omega \Omega^{\infty} S^{\infty} S, C_{1}, M\right) \simeq B\left(\Omega^{\infty} S^{\infty}, C_{1}, M\right)$. Finally, by Thomason [29] and Fiedorowicz [9], $B\left(S, C_{1}, M\right)$ is naturally equivalent to the bar construction $B M$, which yields the equivalence, $\Omega \Omega^{\infty} S^{\infty} B\left(S, C_{1}, M\right) \simeq \Omega \Omega^{\infty} S^{\infty}(B M)$.

When the monoid is a group, we obtain the following important consequence.

Corollary 40 If $\Gamma$ is a group, then $H S_{*}(k[\Gamma]) \cong H_{*}\left(\Omega \Omega^{\infty} S^{\infty}(B \Gamma) ; k\right)$.

Proof From Theorem $38, H S_{*}(k[\Gamma]) \cong H_{*}\left(\underset{\sim}{B}\left(C_{\infty}, C_{1}, \Gamma\right) ; k\right)$. Since $\Gamma$ is a group, $B\left(C_{\infty}, C_{1}, \Gamma\right)$ is grouplike, so $B\left(C_{\infty}, C_{1}, \Gamma\right) \stackrel{\simeq}{\longrightarrow} B\left(\Omega^{\infty} S^{\infty}, C_{1}, \Gamma\right)$. Lemma 39 then applies.

The analysis of $H S_{*}(k[\Gamma])$ then becomes interesting in its own right. We shall have more specific to say about $H S_{1}(k[\Gamma])$ in Section 11.5. 


\section{The category Epi $\Delta S$ and a smaller resolution}

The complexes for computing $H_{*}\left(\Delta S, B_{*}^{\text {sym }} A\right)$ or $H_{*}\left(\Delta S_{+}, B_{*}^{\text {sym }}+A\right)$ are extremely large and unwieldy for computation. Fortunately, when the algebra $A$ is equipped with an augmentation, $\epsilon: A \rightarrow k$, we may use a more manageable subcomplex of $C_{*}\left(\Delta S_{+}, B_{*}^{\mathrm{sym}_{+} A}\right)$, related to the subcategory of $\Delta S_{+}$consisting only of epimorphisms.

\subsection{Basic and reduced tensors}

Recall, if $A$ has an augmentation $\epsilon$, then there is an augmentation ideal $I$ and the exact sequence $0 \longrightarrow I \longrightarrow A \stackrel{\epsilon}{\longrightarrow} k \longrightarrow 0$ splits as $k$-modules. So $A \cong I \oplus k$, and every $x \in A$ can be decomposed uniquely as $x=a+\lambda$ for some $a \in I, \lambda \in k$.

Definition 41 Define $B_{-1, \varnothing} A=k$. For $n \geq 0$, if $J \subseteq[n]$, define

$$
B_{n, J} A \stackrel{\text { def }}{=} B_{0}^{J} \otimes B_{1}^{J} \otimes \cdots \otimes B_{n}^{J}, \quad \text { where } B_{j}^{J}= \begin{cases}I & \text { if } j \in J \\ k & \text { if } j \notin J\end{cases}
$$

Remark 42 For each $n \geq-1$, there is a direct sum decomposition of $k$-modules, $B_{n}^{\mathrm{sym}_{+}} A \cong \bigoplus_{J \subseteq[n]} B_{n, J} A$.

Definition 43 A basic tensor is any tensor $w_{0} \otimes w_{1} \otimes \cdots \otimes w_{n}$, where each $w_{j}$ is in $I$ or is equal to the unit of $A$. Call a tensor factor $w_{j}$ trivial if it is the unit of $A$. If all factors of a basic tensor are trivial, then the tensor is called trivial, and if no factors are trivial, the tensor is called reduced.

For a basic tensor $Y \in B_{n}^{\text {sym }_{+}} A$, we shall define a map $\delta_{Y} \in$ Mor $\Delta_{+}$as follows: If $Y$ is trivial, let $\delta_{Y}=\iota_{n}$. Otherwise, $Y$ has $\bar{n}+1$ non-trivial factors for some $\bar{n} \geq 0$. Define $\delta_{Y}:[\bar{n}] \rightarrow[n]$ to be the unique injective map that sends each point $0,1, \ldots, \bar{n}$ to a point $p \in[n]$ such that $Y$ is non-trivial at factor $p$. Let $\bar{Y}$ be the tensor obtained from $Y$ by omitting all trivial factors if such exist, or $\bar{Y}=1_{k}$ if $Y$ is trivial. Note, $\bar{Y}$ is the unique basic tensor such that $\left(\delta_{Y}\right)_{*}(\bar{Y})=Y$.

Proposition 44 Any chain

$$
\left(\left[q_{n}\right] \leftarrow\left[q_{n-1}\right] \leftarrow \cdots \leftarrow\left[q_{0}\right] \leftarrow[q]\right) \otimes Y \in k\left[N\left(-\backslash \Delta S_{+}\right)\right] \otimes_{\Delta S_{+}} B_{*}^{\mathrm{sym}_{+}} A,
$$

where $Y$ is a basic tensor, is equivalent to a chain

$$
\left(\left[q_{n}\right] \leftarrow\left[q_{n-1}\right] \leftarrow \cdots \leftarrow\left[q_{0}\right] \leftarrow[\bar{q}]\right) \otimes \bar{Y},
$$

where either $\bar{Y}$ is reduced or $\bar{Y}=1_{k}$ and $\bar{q}=-1$. 
Proof Let $\delta_{Y}$ and $\bar{Y}$ be defined as above, and let $[\bar{q}]$ be the domain of $\delta_{Y}$. Then $Y=\left(\delta_{Y}\right)_{*}(\bar{Y})$, and

$$
\left(\left[q_{n}\right] \leftarrow\left[q_{n-1}\right] \leftarrow \cdots \leftarrow\left[q_{0}\right] \stackrel{\phi}{\longleftarrow}[q]\right) \otimes Y \approx\left(\left[q_{n}\right] \leftarrow\left[q_{n-1}\right] \leftarrow \cdots \leftarrow\left[q_{0}\right] \stackrel{\phi \delta_{Y}}{\longleftarrow}[\bar{q}]\right) \otimes \bar{Y}
$$

\subsection{Reducing to epimorphisms}

We now turn our attention to the morphisms in the chains. Our goal is to reduce to those chains that involve only epimorphisms.

Definition 45 Let $\mathscr{C}$ be a category. The category Epi $\mathscr{C}$ (resp. Mono $\mathscr{C}$ ) is the subcategory of $\mathscr{C}$ consisting of the same objects as $\mathscr{C}$ and only those morphisms $f \in \operatorname{Mor}^{\mathscr{C}}$ that are epic (resp. monic). The set of morphisms of Epi $\mathscr{C}$ from $X$ to $Y$ may be denoted $\operatorname{Epi}_{\mathscr{C}}(X, Y)$. Similarly, the set of morphisms of Mono $\mathscr{C}$ from $X$ to $Y$ may be denoted $\operatorname{Mono}_{\mathscr{C}}(X, Y)$.

Remark 46 A morphism $\alpha=(\phi, g) \in$ Mor $\Delta S_{+}$is epic (resp. monic) if and only if $\phi$ is epic (resp. monic) as morphism in $\Delta_{+}$.

Proposition 47 Any morphism $\alpha \in \operatorname{Mor} \Delta S_{+}$decomposes uniquely as ( $\eta$, id) $\circ \gamma$, where $\gamma \in \operatorname{Mor}\left(\operatorname{Epi} \Delta S_{+}\right)$and $\eta \in \operatorname{Mor}\left(\operatorname{Mono} \Delta_{+}\right)$.

Proof Suppose $\alpha$ has source $[-1]$ and target $[n]$. Then $\alpha=\iota_{n}$ is the only possibility, and this decomposes as $\iota_{n} \circ \mathrm{id}_{[-1]}$. Now suppose the source of $\alpha$ is $[p]$ for some $p \geq 0$. Write $\alpha=(\phi, g)$, with $\phi \in \operatorname{Mor}_{\Delta}([p],[r])$ and $g \in \Sigma_{p+1}^{\mathrm{op}}$. Suppose $\phi$ hits $q+1$ distinct points in $[r]$. Then there is a morphism $\pi:[p] \rightarrow[q]$ induced by $\phi$ by maintaining the order of the points hit. Let $\eta$ be the obvious order-preserving monomorphism $[q] \rightarrow[r]$ so that $\eta \pi=\phi$ as morphisms in $\Delta$. To get the required decomposition in $\Delta S$, use: $\alpha=(\eta$, id $) \circ(\pi, g)$.

Now, if $(\xi$, id $) \circ(\psi, h)$ is also a decomposition of $\alpha$, with $\xi$ monic and $\psi$ epic, then $(\xi$, id $) \circ(\psi, h)=(\eta$, id $) \circ(\pi, g)$ implies $\left(\xi \psi, g^{-1} h\right)=(\eta \pi$, id $)$, proving $g=h$. Uniqueness will then follow from uniqueness of such decompositions entirely within the category $\Delta$. The latter follows from Loday [16, Theorem B.2].

This decomposition will be written

$$
[p] \longrightarrow[r]=[p] \rightarrow \operatorname{im}([p] \longrightarrow[r]) \hookrightarrow[r],
$$

and we call the epimorphism construction, the rule

$$
\mathscr{E}_{p}([p] \rightarrow[r])=[p] \rightarrow \operatorname{im}([p] \rightarrow[r]) .
$$


Proposition 48 The epimorphism construction is a functor $\mathscr{E}_{p}:[p] \backslash \Delta S_{+} \rightarrow[p] \backslash$ Epi $\Delta S_{+}$.

Proof If $[p] \stackrel{\beta}{\longrightarrow}\left[r_{1}\right] \stackrel{\alpha}{\longrightarrow}\left[r_{2}\right]$, then there is an induced map

$$
\operatorname{im}\left([p] \longrightarrow\left[r_{1}\right]\right) \stackrel{\bar{\alpha}}{\longrightarrow} \operatorname{im}\left([p] \longrightarrow\left[r_{2}\right]\right)
$$

making the following diagram commute:

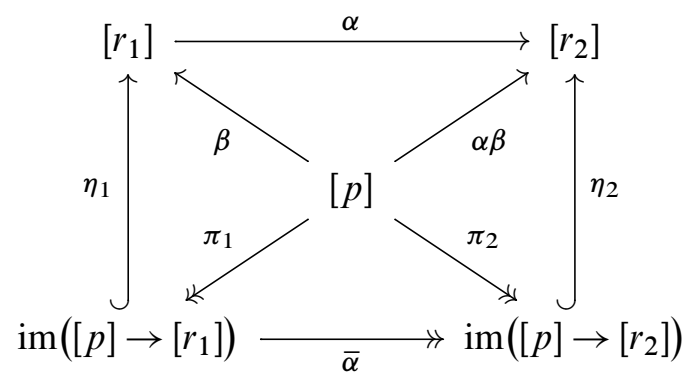

$\bar{\alpha}$ is the epimorphism induced from the map $\alpha \eta_{1}$. Furthermore, for morphisms

$$
[p] \longrightarrow\left[r_{1}\right] \stackrel{\alpha_{1}}{\longrightarrow}\left[r_{2}\right] \stackrel{\alpha_{2}}{\longrightarrow}\left[r_{3}\right]
$$

we have $\overline{\alpha_{2} \alpha_{1}}=\bar{\alpha}_{2} \circ \bar{\alpha}_{1}$.

Remark 49 If $\alpha:[p] \rightarrow[r]$ is an epimorphism of $\Delta S_{+}$, then $\mathscr{E}_{p}(\alpha)=\alpha$.

Define a variant of the symmetric bar construction using Epi $\Delta S$ :

Definition $50 B_{*}^{\mathrm{sym}}+I$ : Epi $\Delta S_{+} \rightarrow k-$ Mod is the functor defined by

$$
\left\{\begin{array}{l}
B_{n}^{\text {sym }_{+}} I=I^{\otimes(n+1)}, \quad n \geq 0, \\
B_{-1}^{\text {sym }_{+}} I=k,
\end{array}\right.
$$

and

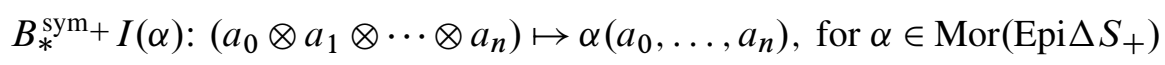

This definition makes sense since $I$ is an ideal, and $\alpha$ is required to be epimorphic. Note, the simple tensors $w_{0} \otimes \cdots \otimes w_{n}$ in $B_{n}^{\mathrm{sym}_{+}} I$ are by definition reduced. Consider the simplicial $k$-module:

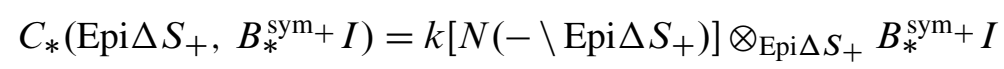


There is an obvious inclusion

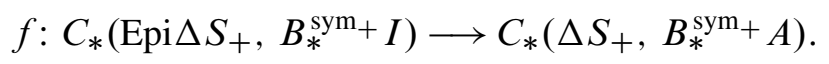

Define a chain map $g$ in the opposite direction as follows. First, by Proposition 44 and observations above, we only need to define $g$ on the chains

$$
\left(\left[q_{n}\right] \longleftarrow \cdots \longleftarrow\left[q_{0}\right] \longleftarrow[q]\right) \otimes Y
$$

where $Y$ is reduced (or $Y=1_{k}$ ). In this case, define component maps $g(q)=$ $N\left(\mathscr{E}_{q}\right) \otimes$ id. A priori, this definition is well-defined only when the tensor product is over $k$. We would like to assemble the maps $g(q)$ into a chain map $g$. In order to do this, we must show that the maps are compatible under $\Delta S_{+}$-equivalence.

Suppose

$$
\begin{aligned}
v & =\left(\left[p_{n}\right] \longleftarrow \cdots \longleftarrow\left[p_{0}\right] \stackrel{\phi \psi}{\longleftarrow}[p]\right) \otimes Z \\
\text { and } \quad & v^{\prime}
\end{aligned}
$$

where $\psi \in \operatorname{Mor}_{\Delta S_{+}}([p],[q])$. If $Z$ is a basic tensor, then so is $\psi_{*}(Z)$. In order to apply $g$ to $v$ or $v^{\prime}$, each must first be put into a reduced form.

Case 1 Suppose $Z$ is trivial. Then $v$ and $v^{\prime}$ both reduce to

$$
\left(\left[p_{n}\right] \longleftarrow \cdots \longleftarrow\left[p_{0}\right] \longleftarrow[-1]\right) \otimes 1,
$$

hence $g(v)=g\left(v^{\prime}\right)$.

Case 2 Suppose $Z$ is non-trivial. For the sake of clean notation, let $W=\psi_{*}(Z)$. Construct $\delta_{Z}, \bar{Z}, \delta_{W}$ and $\bar{W}$ such that $Z=\left(\delta_{Z}\right)_{*}(\bar{Z})$ and $W=\left(\delta_{W}\right)_{*}(\bar{W})$ as in Proposition 44, and reduce both chains:

$$
\begin{array}{cc}
\left(\left[p_{n}\right] \longleftarrow \cdots \longleftarrow[\right. & \left.\left[p_{0}\right] \stackrel{\phi \psi}{\longleftarrow}[p]\right) \otimes Z \\
\text { reduce } \mid \approx & \left(\left[p_{n}\right] \longleftarrow \cdots \longleftarrow\left[p_{0}\right] \stackrel{\phi}{\longleftarrow}[q]\right) \otimes W \\
\left(\left[p_{n}\right] \longleftarrow \cdots \longleftarrow\left[p_{0}\right] \stackrel{\phi \psi \delta_{Z}}{\longleftarrow}[\bar{p}]\right) \otimes \bar{Z} & \left(\left[p_{n}\right] \longleftarrow \cdots \longleftarrow\left[p_{0}\right] \stackrel{\phi \delta_{W}}{\longleftarrow}[\bar{q}]\right) \otimes \bar{W}
\end{array}
$$

Observe that number of distinct points hit by $\psi \delta_{Z}$ is exactly $\bar{q}+1$; indeed, $W=\psi_{*}(Z)$ has $\bar{q}+1$ non-trivial factors. Thus, $[\bar{q}]=\operatorname{im}([\bar{p}] \rightarrow[q])$. Now, Proposition 47 implies that 
there is precisely one $\Delta S$-epimorphism $\gamma:[\bar{p}] \rightarrow[\bar{q}]$ making Diagram (15) commute.

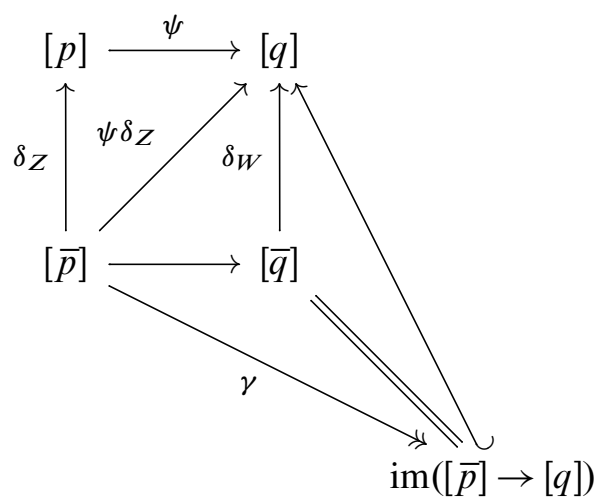

That is to say, there exists an epimorphism $\gamma$ such that $\gamma_{*}(\bar{Z})=\bar{W}$ and $\psi \delta_{Z}=\delta_{W} \gamma$. So we may replace the first morphism of the chain in the lower left of Diagram (14) with $\phi \delta_{W} \gamma$. Then when we apply $g$ to the chain, the first morphism becomes $\mathscr{E} \bar{p}\left(\phi \delta_{W} \gamma\right)=$ $\mathscr{E} \bar{p}\left(\phi \delta_{W}\right) \circ \gamma$, since $\gamma$ is epic. Let $\pi \stackrel{\text { def }_{\mathscr{E}}}{=} \mathscr{E}_{\bar{p}}\left(\phi \delta_{W}\right)$. Then the result of applying $g$ to each side of Diagram (14) is shown below:

$$
\begin{gathered}
\left(\left[p_{n}\right] \longleftarrow \cdots \longleftarrow\left[p_{0}\right] \stackrel{\phi \psi \delta}{\longleftarrow}[\bar{p}]\right) \otimes \bar{Z} \\
g(\bar{p}) \mid \\
\left(\operatorname{im}\left([\bar{p}] \rightarrow\left[p_{n}\right]\right) \longleftarrow \cdots \longleftarrow \operatorname{im}\left([\bar{p}] \rightarrow\left[p_{0}\right]\right) \stackrel{\pi \gamma}{\longleftarrow}[\bar{p}]\right) \otimes \bar{Z} \\
\left(\left[p_{n}\right] \longleftarrow \cdots \longleftarrow\left[p_{0}\right] \stackrel{\phi \delta_{W}}{\longleftarrow}[\bar{q}]\right) \otimes \bar{W} \\
\\
\qquad g(\bar{q}) \\
\left(\operatorname{im}\left([\bar{q}] \rightarrow\left[p_{n}\right]\right) \longleftarrow \cdots \longleftarrow \operatorname{im}\left([\bar{q}] \rightarrow\left[p_{0}\right]\right) \longleftarrow[\bar{q}]\right) \otimes \bar{W}
\end{gathered}
$$

Observe that there is equality of objects and morphisms up to the morphisms $\pi \gamma$ on the left and $\pi$ on the right. Since $\gamma$ is epic, the Epi $\Delta S_{+}$-equivalence allows us to transport the morphism $\gamma$ to the right of the tensor, showing that $g(v) \approx g\left(v^{\prime}\right)$, hence $g$ is well-defined.

Proposition 51 If $A$ has augmentation ideal $I$, then

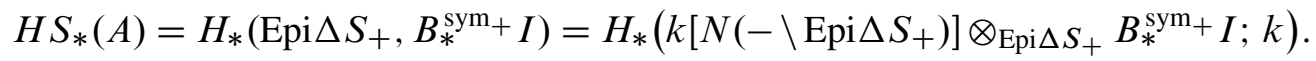


Proof Clearly $g f=\mathrm{id}$, where $f$ is the inclusion mentioned above. It remains to show that $f g \simeq$ id. Assume $Y$ is a basic tensor in $B_{q}^{\text {sym }_{+}} I$. Define maps $h_{j}^{(n)}$ as follows:

$$
\begin{aligned}
h_{j}^{(n)}:\left(\left[q_{n}\right] \leftarrow \cdots \leftarrow\left[q_{0}\right]\right. & \leftarrow[q]) \otimes Y \longmapsto \\
& \left(\left[q_{n}\right] \leftarrow \cdots \leftarrow\left[q_{j}\right] \leftarrow \operatorname{im}\left([q] \rightarrow\left[q_{j}\right]\right) \leftarrow \cdots \leftarrow \operatorname{im}\left([q] \rightarrow\left[q_{0}\right]\right) \leftarrow[q]\right) \otimes Y .
\end{aligned}
$$

$h_{j}^{(n)}$ is well-defined by the functorial properties of the epimorphism construction, and a routine, but tedious, verification shows that $h$ defines a presimplicial homotopy from $f g$ to id.

\subsection{Reduced symmetric homology}

Denote by $B_{*}^{\text {sym }} I$, the restriction of $B_{*}^{\text {sym }}+I$ to $\Delta S$. That is, $B_{n}^{\text {sym }} I \stackrel{\text { def }}{=} I^{\otimes(n+1)}$ for all $n \geq 0$. Then there is a splitting of the chain complex,

$$
C_{*}\left(\operatorname{Epi} \Delta S_{+}, B_{n}^{\mathrm{sym}_{+}} I\right)=C_{*}\left(\operatorname{Epi} \Delta S, B_{n}^{\mathrm{sym}} I\right) \oplus k[N(*)],
$$

where $*$ is the full subcategory of Epi $\Delta S_{+}$consisting only of the object [-1]. Indeed, since there are no epimorphisms $[-1] \rightarrow[n]$ or $[n] \rightarrow[-1]$ for $n \geq 0$, we may think of $[-1]$ as a disconnected basepoint. Hence, we have $H S_{*}(A) \cong H_{*}\left(\right.$ Epi $\left.\Delta S, B_{n}^{\text {sym }} I\right) \oplus$ $k_{0}$, where $k_{0}$ is the graded $k$-module consisting of $k$ concentrated in degree 0 .

Definition 52 The reduced symmetric homology of $A$ is defined,

$$
\tilde{H} S_{*}(A) \stackrel{\text { def }}{=} H_{*}\left(\text { Epi } \Delta S, B_{n}^{\text {sym }} I\right) .
$$

Remark 53 Reduction to epimorphisms seems to hinge on the property that $A$ has an augmentation ideal. This condition may be lifted (as Richter conjectures), if it can be shown that $N($ Epi $\Delta S)$ is contractible. As partial progress along these lines, it can be shown that $N($ Epi $\Delta S)$ is simply-connected.

\section{Interpretation of $H S_{*}(A)$ in terms of group homology}

Epi $\Delta S$ is an E-I-A-category, that is, a category in which all endomorphisms are isomorphisms and also each isomorphism class of an object has only a single member (all isomorphism are automorphisms). In this section, we use results of Słomińska [27] about E-I-A-categories to provide an interpretation of symmetric homology as the homology of products of symmetric groups with coefficients in certain modules. As a corollary, we find that when the ground ring is a field of characteristic $0, H S_{*}(A)$ can be computed as the coinvariants of a group action. 
Fix a unital associative algebra $A$ over commutative ground ring $k$, and assume $A$ has an augmentation, with augmentation ideal $I$. By Proposition 51 and Definition 52,

$$
\tilde{H} S_{*}(A)=H_{*}\left(\text { Epi } \Delta S, B_{*}^{\text {sym }} I\right)=\underset{\text { Epi } \Delta S}{\operatorname{colim}}{ }^{*)} B_{*}^{\text {sym }} I,
$$

where $\operatorname{colim}^{(*)}$ represents the left derived functors of the colimit.

Let $\mathcal{S}_{0}$ be the category whose objects are ordered tuples of non-negative integers, $\left(q_{n}<q_{n-1}<\cdots<q_{0}\right)$, with a unique morphism $\left(q_{n}<\cdots<q_{0}\right) \rightarrow\left(q_{n^{\prime}}^{\prime}<\cdots<q_{0}^{\prime}\right)$ if $\left\{q_{n^{\prime}}^{\prime}, \ldots, q_{0}^{\prime}\right\} \subseteq\left\{q_{n}, \ldots, q_{0}\right\}$. Let $\mathcal{A} u t$ be the functor ( $\mathcal{S}_{0}$-group) defined by

$$
\mathcal{A} u t\left(q_{n}<\cdots<q_{0}\right)=\prod_{i=0}^{n} \Sigma_{q_{i}+1}^{\mathrm{op}}=\prod_{i=0}^{n} \operatorname{Aut}_{\mathrm{EpiS}}\left(\left[q_{i}\right]\right)
$$

Morphisms of $\mathcal{S}_{0}$ map under $\mathcal{A} u t$ to the evident projections. Let $\mathcal{E} p i$ be the functor $\mathcal{S}_{0} \rightarrow$ Set defined by

$$
\mathcal{E} p i\left(q_{n}<\cdots<q_{0}\right)= \begin{cases}\left\{q_{0}\right\}, & \text { if } n=0 \\ \prod_{i=1}^{n} \operatorname{Epi}_{\Delta S}\left(\left[q_{i-1}\right],\left[q_{i}\right]\right), & \text { if } n \geq 1\end{cases}
$$

If $\left\{q_{n^{\prime}}^{\prime}, \ldots, q_{0}^{\prime}\right\} \subseteq\left\{q_{n}, \ldots, q_{0}\right\}$, then the set map

$$
\mathcal{E} p i\left(q_{n}<\cdots<q_{0}\right) \longrightarrow \mathcal{E} p i\left(q_{n^{\prime}}^{\prime}<\cdots<q_{0}^{\prime}\right)
$$

is defined by the appropriate projections and compositions of morphisms, if $n^{\prime} \geq 1$, and by the unique set map into $\left\{q_{0}^{\prime}\right\}$ if $n=0$.

There is an action of $\mathcal{A u t}\left(q_{n}<\cdots<q_{0}\right)$ on $\mathcal{E} p i\left(q_{n}<\cdots<q_{0}\right)$ given by

$$
\mu:\left(g_{n}, \ldots, g_{0}\right) \otimes\left(\phi_{n}, \ldots, \phi_{1}\right) \mapsto\left(g_{n} \phi_{n} g_{n-1}^{-1}, \ldots, g_{1} \phi_{1} g_{0}^{-1}\right)
$$

The maps induced by $\left(q_{n}<\cdots<q_{0}\right) \rightarrow\left(q_{n^{\prime}}^{\prime}<\cdots<q_{0}^{\prime}\right)$ are equivariant with respect to this action in the sense that the following diagram commutes:

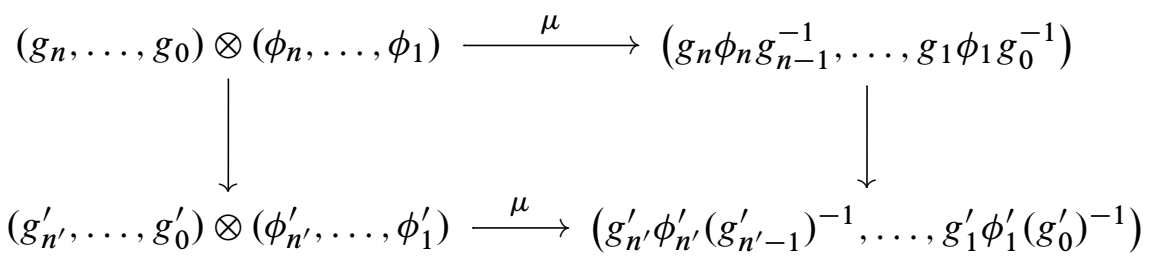

Now, given the augmentation ideal $I$ of $A$, define a related functor, $\mathcal{E} p i_{I}: \mathcal{S}_{0} \rightarrow k-$ Mod by

$$
\left(q_{n}<\cdots<q_{0}\right) \mapsto k\left[\mathcal{E} p i\left(q_{n}<\cdots<q_{0}\right)\right] \otimes_{k} B_{q_{0}}^{\mathrm{sym}} I
$$


The effect of $\mathcal{E} p i_{I}$ on morphisms of $\mathcal{S}_{0}$ requires some explanation. If

$$
\left(q_{n}<\cdots<q_{0}\right) \rightarrow\left(q_{n^{\prime}}^{\prime}<\cdots<q_{0}^{\prime}\right)
$$

is a morphism of $\mathcal{S}_{0}$, then the map

$$
\mathcal{E} p i_{I}\left(q_{n}<\cdots<q_{0}\right) \rightarrow \mathcal{E} p i_{I}\left(q_{n^{\prime}}^{\prime}<\cdots<q_{0}^{\prime}\right)
$$

is defined on generators $\left(\phi_{n}, \ldots, \phi_{1}\right) \otimes x$ by applying the map

$$
\mathcal{E} p i\left(\left(q_{n}<\cdots<q_{0}\right) \rightarrow\left(q_{n^{\prime}}^{\prime}<\cdots<q_{0}^{\prime}\right)\right) \otimes \phi_{r} \cdots \phi_{1},
$$

if $q_{0}^{\prime}=q_{r}$. That is, morphisms "on the left" are thrown out; morphisms "in the middle" are composed; and morphisms "on the right" are applied to the element of $B_{q_{0}}^{\mathrm{sym}} I$ to produce an element of $B_{q_{0}^{\prime}}^{\mathrm{sym}} I$. Each $k\left[\mathcal{E} p i\left(q_{n}<\cdots<q_{0}\right)\right] \otimes B_{q_{0}}^{\mathrm{sym}} I$ becomes an $\mathcal{A u t}\left(q_{n}<\cdots<q_{0}\right)$-module in the obvious way. Diagram (16) implies that there are well-defined functors, $H_{q}\left(\mathcal{A} u t-, \mathcal{E} p i_{I^{-}}\right): \mathcal{S}_{0} \rightarrow k$-Mod.

Theorem 54 With $\mathcal{A} u t$ and $\mathcal{E} p i_{I}$ defined as above, there is a spectral sequence

$$
\operatorname{colim}_{\mathcal{S}_{0}}^{(p)} H_{q}\left(\mathcal{A} u t-, \mathcal{E} p i_{I^{-}}\right) \Rightarrow \tilde{H} S_{p+q}(A)
$$

Proof This is essentially Słomińska [27, Proposition 1.6], reinterpreted in terms of the derived functors of colim. Compare also [27, Proposition 1.8], where variance of the functor $N$ is opposite, and cohomology is computed. Let $Q$ stand for $\left(q_{n}<\cdots<q_{0}\right)$. For each $Q$, let $\mathcal{G} Q$ be the category consisting of a single object whose endomorphism set is the group $\mathcal{A} u t(Q)$. Then we obtain

$$
\begin{aligned}
\operatorname{colim}_{Q \in \mathcal{S}_{0}}{ }^{(p)} H_{q}\left(\mathcal{A u t}(Q), \mathcal{E} p i_{I}(Q)\right)=\operatorname{colim}_{Q \in \mathcal{S}_{0}}{ }^{(p)} \operatorname{colim}_{\mathcal{G} Q}{ }^{(q)} & \left(\mathcal{E} p i_{I}(Q)\right) \\
& \Longrightarrow \operatorname{colim}_{\mathrm{Epi} \Delta S}^{(p+q)} B_{*}^{\mathrm{sym}} I \cong \tilde{H} S_{p+q} A
\end{aligned}
$$

which completes the proof.

\subsection{Implications in characteristic 0}

If $k$ is a field of characteristic 0 , then for any finite group $G$ and $k G$-module $M$, $H_{q}(G, M)=0$ for all $q>0$ (see Brown [1], for example). Thus, the spectral sequence of Theorem 54, would collapse giving the following

Corollary 55 If $k$ is a field of characteristic 0 , and $A$ is a $k$-algebra, then

$$
\tilde{H} S_{p}(A)=\operatorname{colim}_{Q \in \mathcal{S}_{0}}{ }^{(p)} \mathcal{E} p i_{I}(Q)_{\mathcal{A u t}(Q)},
$$


where $\mathcal{E} p i_{I}(Q)_{\mathcal{A u t}(Q)}$ is the $k$-space of coinvariants of $\mathcal{E}_{p i_{I}}(Q)$ under the action of $\operatorname{Aut}(Q)$.

\section{A second spectral sequence}

In Section 7, a spectral sequence was found that essentially separates the symmetric group elements from the morphisms comprising the chains of the resolution $C_{*}\left(\right.$ Epi $\left.\Delta S, B_{*}^{\mathrm{sym}} I\right)$. What remains is still rather cumbersome. The following can be viewed as an attempt to separate the combinatorial structure inherent in the category Epi $\Delta S$ from the more complicated structure generally found in $B_{*}^{\text {sym }} I$. Indeed, the $E^{1}$ term of our spectral sequence isolates the multiplicative structure of $B_{*}^{\mathrm{sym}} I$ into the action of the differential.

\subsection{Filtering by degree}

Consider a filtration $\mathscr{F}_{*}$ of $C_{*}\left(\right.$ Epi $\left.\Delta S, B_{*}^{\text {sym }} I\right)$ given by

$$
\mathscr{F}_{p} C_{q}\left(\text { Epi } \Delta S, B_{*}^{\mathrm{sym}} I\right)=\bigoplus_{p \geq m_{0} \geq \cdots \geq m_{q}} k\left[\prod_{i=1}^{q} \operatorname{Epi}_{\Delta S}\left(\left[m_{i-1}\right],\left[m_{i}\right]\right)\right] \otimes B_{m_{0}}^{\mathrm{sym}} I
$$

$\mathscr{F}_{*}$ filters the complex by the length of $x \in B_{*}^{\text {sym }} I$. Here, length refers to the number of tensor components of $x$. Tensors of $B_{m_{0}}^{\text {sym } I}$ are considered to have length $m_{0}+1$. The only face map that can potentially change the length of $x$ is $d_{0}$, and since all morphisms are epic, $d_{0}$ can only reduce the length. Thus, $\mathscr{F}_{*}$ is compatible with the differential. The filtration quotients are easily described. The non-zero chains are those corresponding to $p=m_{0}$. In other words, the length of $x$ is exactly $p+1 . E^{0}$ splits into a direct sum based on the product of $x_{i}$ 's in $u=\left(x_{0}, \ldots, x_{p}\right) \in X^{p+1}$. For $u \in X^{p+1}$, let $P_{u}$ be the set of all distinct permutations of $u$. Then,

$$
E_{p, q}^{0}=\bigoplus_{u \in X^{p+1} / \Sigma_{p+1}} \bigoplus_{w \in P_{u}}\left(\bigoplus_{p=m_{0} \geq \cdots \geq m_{q}} k\left[\prod_{i=1}^{q} \operatorname{Epi}_{\Delta S}\left(\left[m_{i-1}\right],\left[m_{i}\right]\right)\right] \otimes w\right) .
$$

\subsection{The Categories $\tilde{\mathcal{S}}_{p}, \tilde{\mathcal{S}}_{p}^{\prime}, \mathcal{S}_{p}$ and $\mathcal{S}_{p}^{\prime}$}

Before proceeding with the main theorem of this section, we must define four related categories. In the definitions that follow, let $\left\{z_{0}, z_{1}, z_{2}, \ldots, z_{p}\right\}$ be a set of formal non-commuting indeterminates. 
Definition $56 \widetilde{\mathcal{S}}_{p}$ is the category with objects formal tensor products $Z_{0} \otimes \cdots \otimes Z_{S}$, where each $Z_{i}$ is a non-empty product of $z_{i}$ 's, and every one of $z_{0}, z_{1}, \ldots, z_{p}$ occurs exactly once in the tensor product. There is a unique morphism $Z_{0} \otimes \cdots \otimes Z_{s} \rightarrow$ $Z_{0}^{\prime} \otimes \cdots \otimes Z_{t}^{\prime}$, if and only if the tensor factors of the latter are products of the factors of the former in some order. In such a case, there is a unique $\beta \in \operatorname{Epi} \Delta S$ so that $\beta_{*}\left(Z_{0} \otimes \cdots \otimes Z_{s}\right)=Z_{0}^{\prime} \otimes \cdots \otimes Z_{t}^{\prime}$.

$\widetilde{\mathcal{S}}_{p}$ has initial objects $\sigma_{*}\left(z_{0} \otimes z_{1} \otimes \cdots \otimes z_{p}\right)$, for $\sigma \in \Sigma_{p+1}^{\mathrm{op}}$, so $N \widetilde{\mathcal{S}}_{p}$ is a contractible complex. Let $\widetilde{\mathcal{S}}_{p}^{\prime}$ be the full subcategory of $\widetilde{\mathcal{S}}_{p}$ with all objects $\sigma_{*}\left(z_{0} \otimes \cdots \otimes z_{p}\right)$ deleted.

Let $\mathcal{S}_{p}$ be a skeletal category equivalent to $\widetilde{\mathcal{S}}_{p}$. In fact, we may make $\mathcal{S}_{p}$ the quotient category, identifying each object $Z_{0} \otimes \cdots \otimes Z_{s}$ with any permutation of its tensor factors, and identifying morphisms $\phi$ and $\psi$ if their source and target are equivalent. This category has nerve $N \mathcal{S}_{p}$ homotopy-equivalent to $N \widetilde{\mathcal{S}}_{p}$. Now, $\mathcal{S}_{p}$ is a poset with unique initial object, $z_{0} \otimes \cdots \otimes z_{p}$. Let $\mathcal{S}_{p}^{\prime}$ be the full subcategory (subposet) of $\mathcal{S}_{p}$ obtained by deleting the object $z_{0} \otimes \cdots \otimes z_{p}$. Clearly, $\mathcal{S}_{p}^{\prime}$ is a skeletal category equivalent to $\widetilde{\mathcal{S}}_{p}^{\prime}$.

\subsection{Main theorem}

Theorem 57 There is spectral sequence converging (weakly) to $\tilde{H} S_{*}(A)$ with

$$
E_{p, q}^{1} \cong \bigoplus_{u \in X^{p+1} / \Sigma_{p+1}} H_{p+q}\left(E G_{u} \ltimes_{G_{u}}\left|N \mathcal{S}_{p} / N \mathcal{S}_{p}^{\prime}\right| ; k\right),
$$

where $G_{u}$ is the isotropy subgroup for the chosen representative of $u \in X^{p+1} / \Sigma_{p+1}$.

Recall, for a group $G$, right $G$-space X, and left $G$-space $Y, X \ltimes_{G} Y$ denotes the equivariant half-smash product. If $*$ is a chosen basepoint for $Y$ having trivial $G-$ action, then $X \ltimes_{G} Y \stackrel{\text { def }}{=}\left(X \times_{G} Y\right) /\left(X \times_{G} *\right)=X \times Y / \approx$, with equivalence relation defined by $(x . g, y) \approx(x, g . y)$ and $(x, *) \approx\left(x^{\prime}, *\right)$ for all $x, x^{\prime} \in X, y \in Y$ and $g \in G$ (see Bruner, May, McClure and Steinberger [3]). In our case, $X$ is of the form $E G$, with canonical underlying complex $E_{*} G$, equipped with a right $G$-action, $\left(g_{0}, g_{1}, \ldots, g_{n}\right) \cdot g=\left(g_{0}, g_{1}, \ldots, g_{n} g\right)$.

Observe, both $N \widetilde{\mathcal{S}}_{p}$ and $N \widetilde{\mathcal{S}}_{p}^{\prime}$ carry a left $\Sigma_{p+1}$-action (hence also a $G_{u}$-action). The action is defined on 0 -chains $Z_{0} \otimes \cdots \otimes Z_{s}$ by permutation of the individual indeterminates, $z_{0}, z_{1}, \ldots, z_{p}$. This action extends to $n$-chains in the straightforward manner. 
Define for each $u \in X^{p+1} / \Sigma_{p+1}$, the following subcomplex of $E_{p, q}^{0}$ :

$$
\mathcal{M}_{u} \stackrel{\text { def }}{=} \bigoplus_{w \in P_{u}}\left(\bigoplus_{p=m_{0} \geq \cdots \geq m_{q}} k\left[\prod_{i=1}^{q} \operatorname{Epi}_{\Delta S}\left(\left[m_{i-1}\right],\left[m_{i}\right]\right)\right] \otimes w\right)
$$

Lemma 58 There is a chain-isomorphism, $\left(N \widetilde{\mathcal{S}}_{p} / N \widetilde{\mathcal{S}}_{p}^{\prime}\right) / G_{u} \stackrel{\cong}{\longrightarrow} M_{u}$.

Proof Let $C_{j}$ denote objects of $\widetilde{\mathcal{S}}_{p}$. As above, we may view each $C_{j}$ as a morphism of $\Delta S$. By abuse of notation, let $C_{j}$ also represent a 0 -cell of $N \widetilde{\mathcal{S}}_{p} / N \widetilde{\mathcal{S}}_{p}^{\prime}$. Denote the chosen representative of $u$ again by $u$ (We view $u=\left(x_{i_{0}}, x_{i_{1}}, \ldots, x_{i_{p}}\right) \in X^{p+1} / \Sigma_{p+1}$ as represented by a $(p+1)$-tuple whose indices are in non-decreasing order).

First define a map $N \widetilde{\mathcal{S}}_{p} \longrightarrow \mu_{u}$ on $n$-cells by

$$
\alpha_{*}:\left(C_{q} \stackrel{\phi_{q}}{\longleftarrow} \cdots \stackrel{\phi_{1}}{\longleftarrow} C_{0}\right) \mapsto\left(\phi_{q}, \ldots, \phi_{1}\right) \otimes C_{0}(u) .
$$

The notation $C_{0}(u)$ is used in place of the more correct $\left(C_{0}\right)_{*}(u)$ in order to avoid clutter. I claim $\alpha_{*}$ factors through $N \widetilde{\mathcal{S}}_{p} / N \widetilde{\mathcal{S}}_{p}^{\prime}$. Indeed, if

$$
C_{q} \stackrel{\phi_{q}}{\longleftarrow} \cdots \stackrel{\phi_{1}}{\longleftarrow} C_{0} \in N \widetilde{\mathcal{S}}_{p}^{\prime},
$$

then we cannot have $C_{0}=\sigma\left(z_{0} \otimes \cdots \otimes z_{p}\right)$ for any symmetric group element $\sigma$. That is, $C_{0}$, viewed as a morphism, must be strictly epic. Then the length of of $C_{0}(u)$ is strictly less than the length of $u$, which would make $\left(\phi_{q}, \ldots, \phi_{1}\right) \otimes C_{0}(u)$ trivial in $E_{p, q}^{0}$.

The map $\alpha_{*}$ then factors through $\left(N \widetilde{\mathcal{S}}_{p} / N \widetilde{\mathcal{S}}_{p}^{\prime}\right) / G_{u}$, since if $\gamma \in G_{u}$, then $\gamma$ corresponds to an automorphism $g \in \Sigma_{p+1}^{\mathrm{op}}$, and by definition, we have

$$
\begin{aligned}
\gamma \cdot\left(C_{q} \stackrel{\phi_{q}}{\longleftarrow} \ldots \stackrel{\phi_{1}}{\longleftarrow} C_{0}\right)=\left(C_{q} g \stackrel{\phi_{q}}{\longleftarrow} \ldots \stackrel{\phi_{1}}{\longleftarrow} C_{0} g\right) \longmapsto \\
\\
\left(\phi_{q}, \ldots, \phi_{1}\right) \otimes C_{0}(g(u))=\left(\phi_{q}, \ldots, \phi_{1}\right) \otimes C_{0}(u)
\end{aligned}
$$

(Note, $g(u)=u$ follows from the fact that $\gamma \in G_{u}$, the isotropy subgroup for $u$ ).

For the map in the opposite direction, consider $\left(\phi_{q}, \ldots, \phi_{1}\right) \otimes w$ for $w \in P_{u}$. Let $t \in \Sigma_{p+1}^{\mathrm{op}}$ so that $w=t(u)$. Define a map sending

$$
\beta_{*}:\left(\phi_{q}, \ldots, \phi_{1}\right) \otimes w \mapsto\left(\left(\phi_{q} \cdots \phi_{1} t\right) \stackrel{\phi_{q}}{\longleftarrow} \cdots \stackrel{\phi_{2}}{\longleftarrow} \phi_{1} t \stackrel{\phi_{1}}{\longleftarrow} t\right)
$$


We must check that the definition of $\beta_{*}$ does not depend on choice of $t$. Indeed, if $w=s(u)$ also, then $u=s^{-1} t(u)$, hence $s^{-1} t \in G_{u}^{\text {op }}$. Thus

$$
\begin{aligned}
\left(\left(\phi_{q} \ldots \phi_{1} s\right) \stackrel{\phi_{q}}{\longleftarrow} \cdots \stackrel{\phi_{2}}{\longleftarrow} \phi_{1} s \stackrel{\phi_{1}}{\longleftarrow} s\right) & \approx\left(s^{-1} t\right) \cdot\left(\left(\phi_{q} \cdots \phi_{1} s\right) \stackrel{\phi_{q}}{\longleftarrow} \cdots \stackrel{\phi_{2}}{\longleftarrow} \phi_{1} s \stackrel{\phi_{1}}{\longleftarrow} s\right) \\
& =\left(\left(\phi_{q} \cdots \phi_{1} t\right) \stackrel{\phi_{q}}{\longleftarrow} \cdots \stackrel{\phi_{2}}{\longleftarrow} \phi_{1} t \stackrel{\phi_{1}}{\longleftarrow} t\right)
\end{aligned}
$$

The maps $\alpha_{*}$ and $\beta_{*}$ are clearly inverse to one another. All that remains is to verify that they are chain maps. We need only check compatibility with the zeroth face maps in either case, since the $i$ th face maps (for $i>0$ ) simply compose the morphisms $\phi_{i+1}$ and $\phi_{i}$ in either chain complex. The zeroth face maps of either complex will be denoted $d_{0}$.

First consider the map $\alpha_{*}$.

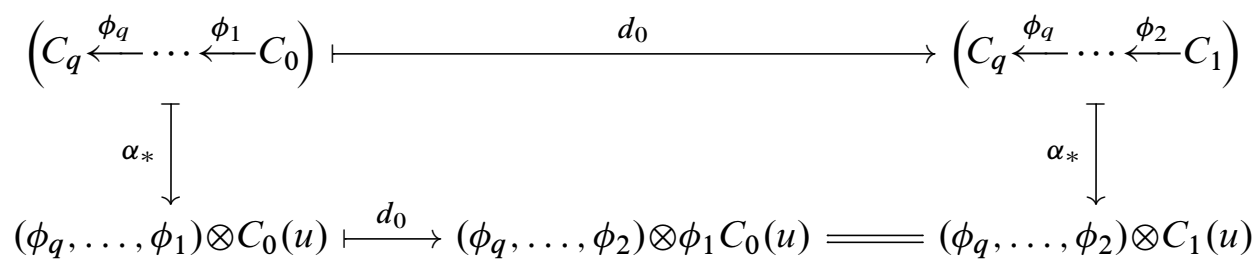

The equality in the lower right of the diagram is simply a restatement that $C_{0} \stackrel{\phi_{1}}{\rightarrow} C_{1}$ is a morphism of $\widetilde{\mathcal{S}}_{p}$.

For the reverse direction, assume $w=t(u)$ as above. The following diagram commutes.

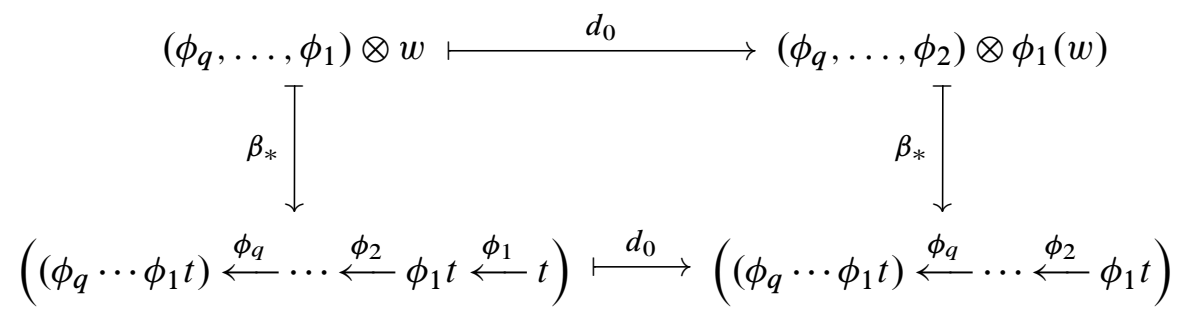

The lower righthand corner deserves some explanation. Since the target of $\beta_{*}$ should be $\left(N \widetilde{\mathcal{S}}_{p} / N \widetilde{\mathcal{S}}_{p}^{\prime}\right) / G_{u}$, we see that the lower righthand term is trivial unless $\phi_{1}$ is an isomorphism. In other words, we only need to prove the diagram commutes when $\phi_{1} \in \Sigma_{p+1}^{\mathrm{op}}$. As $\beta_{*}$ is applied to the chain on the upper righthand corner, the clear choice for group element to begin the chain is $\phi_{1} t$, since $\phi_{1}(w)=\phi_{1} t(u)$. 
Using Lemma 58 , we identify $\mu_{u}$ with the orbit complex $\left(N \widetilde{\mathcal{S}}_{p} / N \widetilde{\mathcal{S}}_{p}^{\prime}\right) / G_{u}$. Now, the complex $N \widetilde{\mathcal{S}}_{p} / N \widetilde{\mathcal{S}}_{p}^{\prime}$ is a free $G_{u}$-complex, so we have an isomorphism,

$$
H_{*}\left(\left(N \widetilde{\mathcal{S}}_{p} / N \widetilde{\mathcal{S}}_{p}^{\prime}\right) / G_{u}\right) \cong H_{*}^{G_{u}}\left(N \widetilde{\mathcal{S}}_{p} / N \widetilde{\mathcal{S}}_{p}^{\prime}\right)
$$

where $H_{*}^{G_{u}}$ is $G_{u}$-equivariant homology. See Brown [1] for details). Then, by definition,

$$
H_{*}^{G_{u}}\left(N \widetilde{\mathcal{S}}_{p} / N \widetilde{\mathcal{S}}_{p}^{\prime}\right)=H_{*}\left(G_{u}, N \widetilde{\mathcal{S}}_{p} / N \widetilde{\mathcal{S}}_{p}^{\prime}\right),
$$

which may be computed using the free resolution, $E_{*} G_{u}$ of $k$ as right $G_{u}$-module. The resulting complex $k\left[E_{*} G_{u}\right] \otimes_{k G_{u}} k\left[N \widetilde{\mathcal{S}}_{p}\right] / k\left[N \widetilde{\mathcal{S}}_{p}^{\prime}\right]$ is a double complex isomorphic to the quotient

$$
\begin{aligned}
\left(k\left[E_{*} G_{u}\right] \otimes_{k G_{u}} k\left[N \widetilde{\mathcal{S}}_{p}\right]\right) /\left(k\left[E_{*} G_{u}\right]\right. & \left.\otimes_{k G_{u}} k\left[N \widetilde{\mathcal{S}}_{p}^{\prime}\right]\right) \\
& \cong k\left[\left(E_{*} G_{u} \times G_{u} N \widetilde{\mathcal{S}}_{p}\right) /\left(E_{*} G_{u} \times G_{u} N \widetilde{\mathcal{S}}_{p}^{\prime}\right)\right] .
\end{aligned}
$$

This last complex may be identified with the simplicial complex of the space,

$$
\left(E G_{u} \times_{G_{u}}\left|N \widetilde{\mathcal{S}}_{p}\right|\right) /\left(E G_{u} \times_{G_{u}}\left|N \widetilde{\mathcal{S}}_{p}^{\prime}\right|\right) \cong E G_{u} \ltimes_{G_{u}}\left|N \widetilde{\mathcal{S}}_{p} / N \widetilde{\mathcal{S}}_{p}^{\prime}\right|
$$

The last piece of the puzzle involves simplifying the spaces $\left|N \widetilde{\mathcal{S}}_{p} / N \widetilde{\mathcal{S}}_{p}^{\prime}\right|$. Since $\mathcal{S}$ is a skeletal subcategory of $\widetilde{\mathcal{S}}$, there is an equivalence of categories $\widetilde{\mathcal{S}} \simeq \mathcal{S}$, inducing a homotopy equivalence of complexes (hence also of spaces) $|N \widetilde{\mathcal{S}}| \simeq|N \mathcal{S}|$. Note that $N \mathcal{S}$ inherits a $G_{u}$-action from $N \widetilde{\mathcal{S}}$, and the map $\widetilde{\mathcal{S}} \rightarrow \mathcal{S}$ is $G_{u}$-equivariant.

Proposition 59 There are weak equivalences, $E G_{u} \times_{G_{u}}\left|N \widetilde{\mathcal{S}}_{p}\right| \rightarrow E G_{u} \times_{G_{u}}\left|N \mathcal{S}_{p}\right|$ and $E G_{u} \times G_{u}\left|N \widetilde{\mathcal{S}}_{p}^{\prime}\right| \rightarrow E G_{u} \times G_{u}\left|N \mathcal{S}_{p}^{\prime}\right|$, inducing a weak equivalence $E G_{u} \ltimes_{G_{u}}$ $\left|N \widetilde{\mathcal{S}}_{p} / N \widetilde{\mathcal{S}}_{p}^{\prime}\right| \rightarrow E G_{u} \ltimes_{G_{u}}\left|N \mathcal{S}_{p} / N \mathcal{S}_{p}^{\prime}\right|$.

Proof The case $p>2$ will be handled first. As long as the spaces are path connected, we can use the fibration sequences associated to $X \rightarrow E G_{u} \times{ }_{G_{u}} X \rightarrow B G_{u}$ to show that a $G_{u}$-equivariant homotopy equivalence $X \simeq Y$ induces $E G_{u} \times{ }_{G_{u}} X \simeq E G_{u} \times{ }_{G_{u}} Y$. $\left|N \widetilde{\mathcal{S}}_{p}\right|$ and $\left|N \mathcal{S}_{p}\right|$ are path-connected because they are contractible. It suffices to show $\left|N \mathcal{S}_{p}^{\prime}\right|$ path connected, since $\left|N \widetilde{\mathcal{S}}_{p}^{\prime}\right| \simeq\left|N \mathcal{S}_{p}^{\prime}\right|$.

let $W_{0}=z_{0} z_{1} \otimes z_{2} \otimes \cdots \otimes z_{p}$. This represents a vertex of $N \mathcal{S}_{p}^{\prime}$. Suppose

$$
W=Z_{0} \otimes \cdots \otimes Z_{i}^{\prime} z_{0} z_{1} Z_{i}^{\prime \prime} \otimes \cdots \otimes Z_{s} .
$$

Then there is a morphism $W_{0} \rightarrow W$, hence an edge between $W_{0}$ and $W$. 
Next, suppose $W=Z_{0} \otimes \cdots \otimes Z_{i}^{\prime} z_{0} Z_{i}^{\prime \prime} z_{1} Z_{i}^{\prime \prime \prime} \otimes \cdots \otimes Z_{s}$. There is a path

$$
\begin{gathered}
Z_{0} \otimes \cdots \otimes Z_{i}^{\prime} z_{0} Z_{i}^{\prime \prime} z_{1} Z_{i}^{\prime \prime \prime} \otimes \cdots \otimes Z_{s} \\
Z_{0} Z_{1} \ldots Z_{i}^{\prime} z_{0} Z_{i}^{\prime \prime} z_{1} Z_{i}^{\prime \prime \prime} \ldots Z_{s} \\
\sum_{Z_{0} Z_{1} \ldots Z_{i}^{\prime} \otimes z_{0}} \otimes Z_{i}^{\prime \prime} z_{1} Z_{i}^{\prime \prime \prime} \ldots Z_{s} \\
\left.\right|_{z_{0} \otimes Z_{0} Z_{1} \ldots Z_{i}^{\prime} Z_{i}^{\prime \prime} z_{1} Z_{i}^{\prime \prime \prime} \ldots Z_{s}} ^{\uparrow} \\
\left.\right|_{z_{0} \otimes Z_{0} Z_{1} \ldots Z_{i}^{\prime} Z_{i}^{\prime \prime} \otimes z_{1} Z_{i}^{\prime \prime \prime} \ldots Z_{s}} \\
\left.\right|_{z_{0} z_{1} Z_{i}^{\prime \prime \prime} \ldots Z_{s} \otimes} Z_{0} Z_{1} \ldots Z_{i}^{\prime} Z_{i}^{\prime \prime} \\
W_{0} .
\end{gathered}
$$

Similarly, if $W=Z_{0} \otimes \cdots \otimes Z_{i}^{\prime} z_{1} Z_{i}^{\prime \prime} z_{0} Z_{i}^{\prime \prime \prime} \otimes \cdots \otimes Z_{s}$, there is a path to $W_{0}$. Finally, if $W=Z_{0} \otimes \cdots \otimes Z_{s}$ with $z_{0}$ occurring in $Z_{i}$ and $z_{1}$ occurring in $Z_{j}$ for $i \neq j$, there is an edge to some $W^{\prime}$ in which $Z_{i} Z_{j}$ occurs, and thus a path to $W_{0}$.

The cases $p=0,1$ and 2 are handled individually:

Observe that $\left|N \widetilde{\mathcal{S}}_{0}^{\prime}\right|$ and $\left|N \mathcal{S}_{0}^{\prime}\right|$ are empty spaces, since $\widetilde{\mathcal{S}}_{0}^{\prime}$ has no objects. Hence

$$
E G_{u} \times_{G_{u}}\left|N \widetilde{\mathcal{S}}_{0}^{\prime}\right|=E G_{u} \times{ }_{G_{u}}\left|N \mathcal{S}_{0}^{\prime}\right|=\varnothing .
$$

Furthermore, any group $G_{u}$ must be trivial. Thus there is a chain of homotopy equivalences

$$
E G_{u} \ltimes_{G_{u}}\left|N \widetilde{\mathcal{S}}_{0} / N \widetilde{\mathcal{S}}_{0}^{\prime}\right| \simeq\left|N \widetilde{\mathcal{S}}_{0}\right| \simeq\left|N \mathcal{S}_{0}\right| \simeq E G_{u} \ltimes_{G_{u}}\left|N \mathcal{S}_{p} / N \mathcal{S}_{p}^{\prime}\right| .
$$

Next, since $\left|N \widetilde{\mathcal{S}}_{1}^{\prime}\right|$ is homeomorphic to $\left|N \mathcal{S}_{1}^{\prime}\right|$, each space consisting of the two discrete points $z_{0} z_{1}$ and $z_{1} z_{0}$ with the same group action, the proposition is true for $p=1$ as well. 
For $p=2$, observe that $\left|N \widetilde{\mathcal{S}}_{2}^{\prime}\right|$ has two connected components, $\tilde{U}_{1}$ and $\tilde{U}_{2}$ that are interchanged by any odd permutation $\sigma \in \Sigma_{3}$. Similarly, $\left|N \mathcal{S}_{2}^{\prime}\right|$ consists of two connected components, $U_{1}$ and $U_{2}$, interchanged by any odd permutation of $\Sigma_{3}$. Now, resticted to the alternating group, $A_{3}$, we certainly have weak equivalences for any subgroup $H_{u} \subseteq A_{3}$,

$$
E H_{u} \times_{H_{u}} \tilde{U}_{1} \stackrel{\simeq}{\longrightarrow} E H_{u} \times_{H_{u}} U_{1} \quad \text { and } \quad E H_{u} \times_{H_{u}} \tilde{U}_{2} \stackrel{\simeq}{\longrightarrow} E H_{u} \times H_{u} U_{2} .
$$

The action of an odd permutation induces equivariant homeomorphisms $\widetilde{U}_{1} \cong \widetilde{U}_{2}$ and $U_{1} \stackrel{\cong}{\longrightarrow} U_{2}$, and so if we have a subgroup $G_{u} \subseteq \Sigma_{3}$ generated by $H_{u} \subseteq A_{3}$ and a transposition, then the two connected components are identified in an $A \Sigma_{3}$-equivariant manner. Thus, if $G_{u}$ contains a transposition,

$$
E G_{u} \times_{G_{u}}\left|N \widetilde{\mathcal{S}}_{2}^{\prime}\right| \cong E H_{u} \times_{H_{u}} \tilde{U}_{1} \simeq E H_{u} \times_{H_{u}} U_{1} \cong E G_{u} \times_{G_{u}}\left|N \mathcal{S}_{2}^{\prime}\right|
$$

This completes the case $p=2$ and the proof of Proposition 59 .

Proposition 59 coupled with Lemma 58 produces the required isomorphism in homology, hence proving Theorem 57:

$$
\begin{aligned}
E_{p, q}^{1}=\bigoplus_{u \in X^{p+1} / \Sigma_{p+1}} H_{p+q}\left(\mathcal{M}_{u}\right) & \cong \bigoplus_{u \in X^{p+1} / \Sigma_{p+1}} H_{p+q}\left(E G_{u} \ltimes_{G_{u}}\left|N \widetilde{\mathcal{S}}_{p} / N \widetilde{\mathcal{S}}_{p}^{\prime}\right| ; k\right) \\
& \cong \bigoplus_{u \in X^{p+1} / \Sigma_{p+1}} H_{p+q}\left(E G_{u} \ltimes_{G_{u}}\left|N \mathcal{S}_{p} / N \mathcal{S}_{p}^{\prime}\right| ; k\right)
\end{aligned}
$$

Corollary 60 If the augmentation ideal of $A$ satisfies $I^{2}=0$, then

$$
\tilde{H} S_{n}(A) \cong \bigoplus_{p \geq 0} \bigoplus_{u \in X^{p+1} / \Sigma_{p+1}} H_{n}\left(E G_{u} \ltimes_{G_{u}} N \mathcal{S}_{p} / N \mathcal{S}_{p}^{\prime} ; k\right)
$$

Proof This follows from consideration of the original $E^{0}$ term of the spectral sequence. $E^{0}$ is generated by chains $\left(\phi_{n}, \ldots, \phi_{1}\right) \otimes w$ with induced differential $d^{0}$, agreeing with the differential $d$ of $C_{*}\left(\right.$ Epi $\left.\Delta S, B_{*}^{\text {sym }} I\right)$ when $\phi_{0}$ is an isomorphism. When $\phi_{0}$ is a strict epimorphism, however, the zeroth face map of $d$ maps the generator to: $\left(\phi_{n}, \ldots, \phi_{1}\right) \otimes\left(\phi_{0}\right)_{*}(Y)=0$, since $\left(\phi_{0}\right)_{*}(Y)$ would have at least one tensor factor that is the product of two or more elements of $I$. Thus, $d^{0}$ also agrees with $d$ in the case that $\phi_{0}$ is strictly epic. Hence, the spectral sequence collapses at level 1 . 


\subsection{The complex $\operatorname{Sym}_{*}^{(p)}$}

Note, for $p>0$, there are homotopy equivalences $\left|N \mathcal{S}_{p} / N \mathcal{S}_{p}^{\prime}\right| \simeq\left|N \mathcal{S}_{p}\right| \vee S\left|N \mathcal{S}_{p}^{\prime}\right| \simeq$ $S\left|N \mathcal{S}_{p}^{\prime}\right|$, since $\left|N \mathcal{S}_{p}\right|$ is contractible. $\left|N \mathcal{S}_{p}\right|$ is a disjoint union of $(p+1)$ ! $p$-cubes, identified along certain faces. Geometric analysis of $S\left|N \mathcal{S}_{p}^{\prime}\right|$, however, seems quite difficult. Fortunately, there is an even smaller chain complex homotopic to $N \mathcal{S}_{p} / N \mathcal{S}_{p}^{\prime}$.

Definition 61 Let $p \geq 0$ and impose an equivalence relation on $k\left[\operatorname{Epi}_{\Delta S}([p],[q])\right]$ generated by

$$
Z_{0} \otimes \cdots \otimes Z_{i} \otimes Z_{i+1} \otimes \cdots \otimes Z_{q} \approx(-1)^{a b} Z_{0} \otimes \cdots \otimes Z_{i+1} \otimes Z_{i} \otimes \cdots \otimes Z_{q},
$$

where $Z_{0} \otimes \cdots \otimes Z_{q}$ is a morphism expressed in tensor notation, and $a=\operatorname{deg}\left(Z_{i}\right)$ and $b=\operatorname{deg}\left(Z_{i+1}\right)$, where $\operatorname{deg}(Z) \stackrel{\text { def }}{=}|Z|-1$, one less than the number of tensor factors of $Z$. The complex $\operatorname{Sym}_{*}^{(p)}$ is then defined by

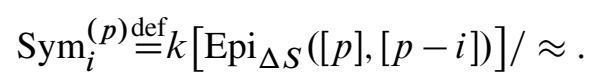

The face maps will be defined recursively. On monomials,

$$
d_{i}\left(z_{j_{0}} \ldots z_{j_{s}}\right)= \begin{cases}0, & i<0 \\ z_{j_{0}} \ldots z_{j_{i}} \otimes z_{j_{i+1}} \ldots z_{j_{s}}, & 0 \leq i<s \\ 0, & i \geq s\end{cases}
$$

Then, extend $d_{i}$ to tensor products via

$$
d_{i}(W \otimes V)=d_{i}(W) \otimes V+W \otimes d_{i-\operatorname{deg}(W)}(V),
$$

where $W$ and $V$ are formal tensors in $k\left[\operatorname{Epi}_{\Delta S}([p],[q])\right]$, and

$$
\operatorname{deg}(W)=\operatorname{deg}\left(W_{0} \otimes \cdots \otimes W_{t}\right) \stackrel{\text { def }}{=} \sum_{k=0}^{t} \operatorname{deg}\left(W_{k}\right) .
$$

The boundary map $\operatorname{Sym}_{n}^{(p)} \rightarrow \operatorname{Sym}_{n-1}^{(p)}$ is then $d=\sum_{i=0}^{n}(-1)^{i} d_{i}=\sum_{i=0}^{n-1}(-1)^{i} d_{i}$.

Remark 62 There is an action $\Sigma_{p+1} \times \operatorname{Sym}_{i}^{(p)} \rightarrow \operatorname{Sym}_{i}^{(p)}$, given by permuting the formal indeterminates $z_{i}$. Furthermore, this action is compatible with the differential.

Lemma $63 \operatorname{Sym}_{*}^{(p)}$ is homotopy-equivalent to $k\left[N \mathcal{S}_{p}\right] / k\left[N \mathcal{S}_{p}^{\prime}\right]$.

Proof Let $v_{0}$ represent the common initial vertex of the $p$-cubes making up $N \mathcal{S}_{p}$. Then, as cell-complex, $N \mathcal{S}_{p}$ consists of $v_{0}$ together with all corners of the various $p$-cubes and $i$-cells for each $i$-face of the cubes. Thus, $N \mathcal{S}_{p}$ consists of $(p+1)$ ! 
$p$-cells with attaching maps $\partial I^{p} \rightarrow\left(N \mathcal{S}_{p}\right)^{p-1}$ defined according to the face maps for $N \mathcal{S}_{p}$ given above. Note that a chain of $N \mathcal{S}_{p}$ is non-trivial in $N \mathcal{S}_{p} / N \mathcal{S}_{p}^{\prime}$ if and only if the initial vertex $v_{0}$ is included. Thus, any (cubical) $k$-cell is uniquely determined by the label of the vertex opposite $v_{0}$.

Label each top-dimensional cell with the permutation induced on the set $\{0,1, \ldots, p\}$ by the order of indeterminates in its final vertex, $z_{i_{0}} z_{i_{1}} \ldots z_{i_{p}}$. On a given $p$-cell, for each vertex $Z_{0} \otimes \cdots \otimes Z_{s}$, there is an ordering of the tensor factors so that $Z_{0} \otimes \cdots \otimes Z_{s} \rightarrow z_{i_{0}} z_{i_{1}} \ldots z_{i_{p}}$ preserves the order of formal indeterminates $z_{i}$. Rewrite each vertex of this $p$-cell in this order. Now, any $p$-chain $\left(z_{i_{0}} \otimes z_{i_{1}} \otimes \cdots \otimes z_{i_{p}}\right) \rightarrow$ $\cdots \rightarrow z_{i_{0}} z_{i_{1}} \ldots z_{i_{p}}$ is obtained by choosing the order in which to combine the factors. In fact, the $p$-chains for this cube are in bijection with the elements of the symmetric group $S_{p}$, as in the standard decomposition of a $p$-cube into $p$ ! simplices. A given permutation $\{1,2, \ldots, p\} \mapsto\left\{j_{1}, j_{2}, \ldots, j_{p}\right\}$ will represent the chain obtained by first combining $z_{j_{0}} \otimes z_{j_{1}}$ into $z_{j_{0}} z_{j_{1}}$, then combining $z_{j_{1}} \otimes z_{j_{2}}$ into $z_{j_{1}} z_{j_{2}}$. In effect, we "erase" the tensor product symbol between $z_{j_{r-1}}$ and $z_{j_{r}}$ for each $j_{r}$ in order given by the list above.

We shall declare that the natural order of combining the factors will be the one that always combines the last two:

$$
\begin{aligned}
\left(z_{i_{0}} \otimes \cdots \otimes z_{i_{p-1}} \otimes z_{i_{p}}\right) \longrightarrow\left(z_{i_{0}} \otimes \cdots \otimes z_{i_{p-1}} z_{i_{p}}\right) \longrightarrow \\
\quad\left(z_{i_{0}} \otimes \cdots \otimes z_{i_{p-2}} z_{i_{p-1}} z_{i_{p}}\right) \longrightarrow \cdots \longrightarrow\left(z_{i_{0}} \ldots z_{i_{p}}\right) .
\end{aligned}
$$

This corresponds to a permutation $\rho \stackrel{\text { def }}{=}\{1, \ldots, p\} \mapsto\{p, p-1, \ldots, 2,1\}$, and this chain will be regarded as positive. A chain $C_{\sigma}$, corresponding to another permutation, $\sigma$, will be regarded as positive or negative depending on the sign of the permutation $\sigma \rho^{-1}$. Finally, the entire $p$-cell should be identified with the sum $\sum_{\sigma \in S_{p}} \operatorname{sgn}\left(\sigma \rho^{-1}\right) C_{\sigma}$. It is this sign convention that permits the inner faces of the cube to cancel appropriately in the boundary maps. Thus we have a map on the top-dimensional chains:

$$
\theta_{p}: \operatorname{Sym}_{p}^{(p)} \rightarrow\left(k\left[N \mathcal{S}_{p}\right] / k\left[N \mathcal{S}_{p}^{\prime}\right]\right)_{p}
$$

Extend the defintion $\theta_{*}$ to arbitrary $k$-cells by sending the $k$-chain $Z_{0} \otimes \cdots \otimes Z_{p-k}$ to the sum of $k$-length chains with source $z_{0} \otimes \cdots \otimes z_{p}$ and target $Z_{0} \otimes \cdots \otimes Z_{p-k}$ with signs determined by the natural order of erasing tensor product symbols of $z_{0} \otimes \cdots \otimes z_{p}$, excluding those tensor product symbols that never get erased. The following example should clarify the point. Let $W=z_{3} z_{0} \otimes z_{1} \otimes z_{2} z_{4}$. This is a 2-cell of $\operatorname{Sym}_{*}^{(4)} . W$ is obtained from $z_{0} \otimes z_{1} \otimes z_{2} \otimes z_{3} \otimes z_{4}=z_{3} \otimes z_{0} \otimes z_{1} \otimes z_{2} \otimes z_{4}$ by combining factors in some order. There are only 2 erasable tensor product symbols in this example. The natural order (last to first) corresponds to the chain, $z_{3} \otimes z_{0} \otimes z_{1} \otimes z_{2} \otimes z_{4} \rightarrow$ 
$z_{3} \otimes z_{0} \otimes z_{1} \otimes z_{2} z_{4} \rightarrow z_{3} z_{0} \otimes z_{1} \otimes z_{2} z_{4}$. So, this chain shows up in $\theta_{*}(W)$ with positive sign, whereas the chain $z_{3} \otimes z_{0} \otimes z_{1} \otimes z_{2} \otimes z_{4} \rightarrow z_{3} z_{0} \otimes z_{1} \otimes z_{2} \otimes z_{4} \rightarrow z_{3} z_{0} \otimes z_{1} \otimes z_{2} z_{4}$ shows up with a negative sign.

Now, $\theta_{*}$ is easily seen to be a chain map $\operatorname{Sym}_{*}^{(p)} \rightarrow k\left[N \mathcal{S}_{p}\right] / k\left[N \mathcal{S}_{p}^{\prime}\right]$. Geometrically, $\theta_{*}$ has the effect of subdividing a cell-complex (defined with cubical cells) into a simplicial space, so $\theta_{*}$ is a homotopy-equivalence. Furthermore, $\theta_{*}$ is equivariant with respect to the action of the symmetric group.

Remark 64 As an example, consider $\left|N \mathcal{S}_{2}\right|$. There are 62 -cells, each represented by a copy of $I^{2}$. The $2-$ cell labelled by the permutation $\{0,1,2\} \mapsto\{1,0,2\}$ consists of the chains $z_{1} \otimes z_{0} \otimes z_{2} \rightarrow z_{1} \otimes z_{0} z_{2} \rightarrow z_{1} z_{0} z_{2}$ and $-\left(z_{1} \otimes z_{0} \otimes z_{2} \rightarrow z_{1} z_{0} \otimes z_{2} \rightarrow z_{1} z_{0} z_{2}\right)$. Hence, the boundary is the sum of 1 -chains:

$$
\begin{aligned}
& {\left[\left(z_{1} \otimes z_{0} z_{2} \rightarrow z_{1} z_{0} z_{2}\right)-\left(z_{1} \otimes z_{0} \otimes z_{2} \rightarrow z_{1} z_{0} z_{2}\right)+\left(z_{1} \otimes z_{0} \otimes z_{2} \rightarrow z_{1} \otimes z_{0} z_{2}\right)\right]} \\
& \quad-\left[\left(z_{1} z_{0} \otimes z_{2} \rightarrow z_{1} z_{0} z_{2}\right)-\left(z_{1} \otimes z_{0} \otimes z_{2} \rightarrow z_{1} z_{0} z_{2}\right)+\left(z_{1} \otimes z_{0} \otimes z_{2} \rightarrow z_{1} z_{0} \otimes z_{2}\right)\right] \\
& =\left(z_{1} \otimes z_{0} z_{2} \rightarrow z_{1} z_{0} z_{2}\right)+\left(z_{1} \otimes z_{0} \otimes z_{2} \rightarrow z_{1} \otimes z_{0} z_{2}\right)\left(z_{1} z_{0} \otimes z_{2} \rightarrow z_{1} z_{0} z_{2}\right) \\
& \quad-\left(z_{1} \otimes z_{0} \otimes z_{2} \rightarrow z_{1} z_{0} \otimes z_{2}\right) .
\end{aligned}
$$

These 1-chains correspond to the 4 edges of the square. Thus, in our example this 2 -cell of $\left|N \mathcal{S}_{p}\right|$ will correspond to $z_{1} z_{0} z_{2} \in \mathrm{Sym}_{2}^{(2)}$, and its boundary in $\left|N \mathcal{S}_{p} / N \mathcal{S}_{p}^{\prime}\right|$ will consist of the two edges adjacent to the vertex labeled $z_{0} \otimes z_{1} \otimes z_{2}$, with appropriate signs: $\left(z_{0} \otimes z_{1} \otimes z_{2} \rightarrow z_{1} \otimes z_{0} z_{2}\right)-\left(z_{0} \otimes z_{1} \otimes z_{2} \rightarrow z_{1} z_{0} \otimes z_{2}\right)$. The corresponding boundary in $\operatorname{Sym}_{1}^{(2)}$ will be $\left(z_{1} \otimes z_{0} z_{2}\right)-\left(z_{1} z_{0} \otimes z_{2}\right)$, matching the boundary map already defined on $\operatorname{Sym}_{*}^{(p)}$. See Figures 3 and 4 .

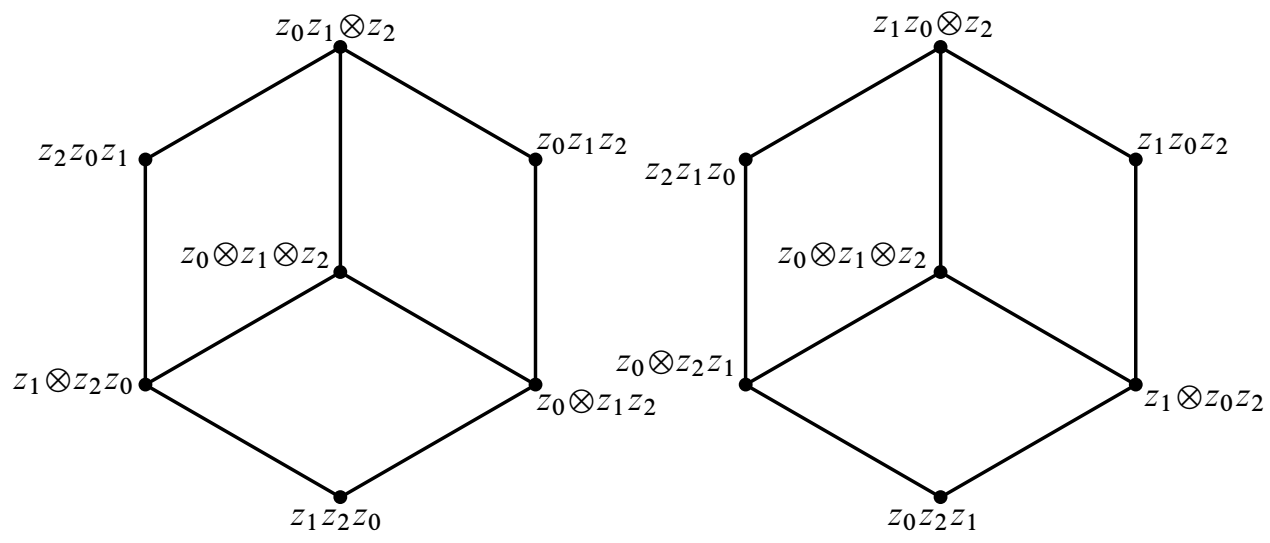

Figure 3: $\left|N \mathcal{S}_{2}\right|$ consists of six squares, grouped into two hexagons that share a common center vertex 

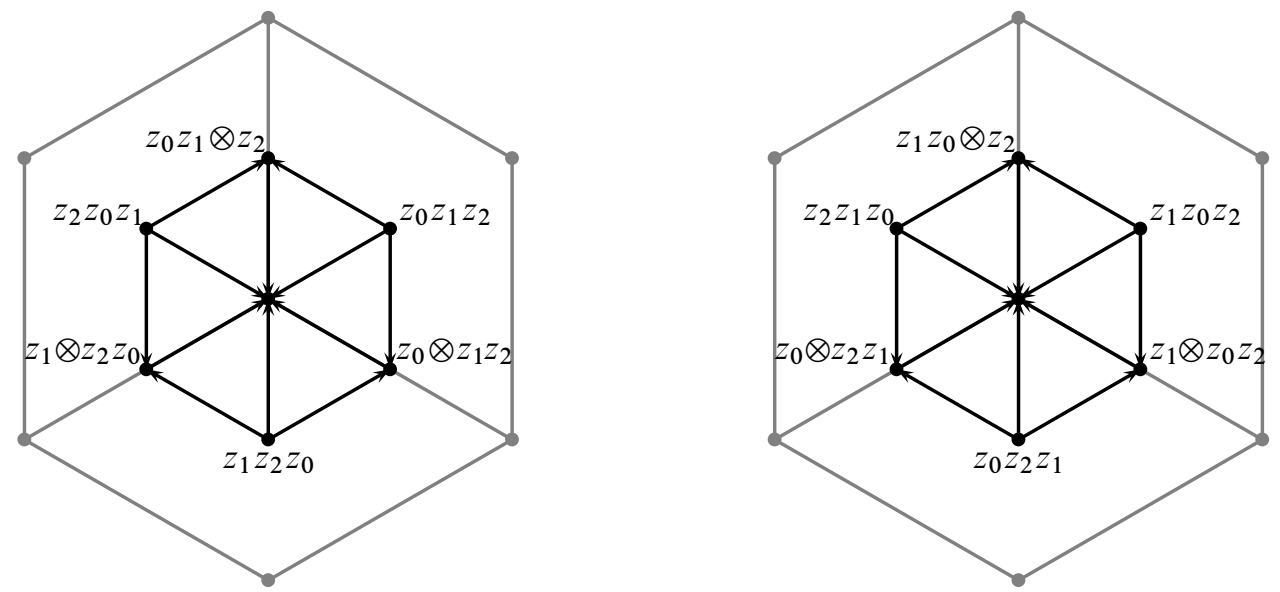

Figure 4: $\operatorname{Sym}^{(2)} \simeq N \mathcal{S}_{2} / N \mathcal{S}_{2}^{\prime}$. The center of each hexagon is $z_{0} \otimes z_{1} \otimes z_{2}$.

Now, with one piece of new notation, we may re-interpret Theorem 57.

Definition 65 Let $G$ be a group. Let $k_{0}$ be the chain complex consisting of $k$ concentrated in degree 0 , with trivial $G$-action. If $X_{*}$ is a right $G$-complex, $Y_{*}$ is a left $G$-complex with $k_{0} \hookrightarrow Y_{*}$ as a $G$-subcomplex, then define the equivariant half-smash tensor product of the two complexes:

$$
X_{*} \otimes_{G} Y_{*} \stackrel{\text { def }}{=}\left(X_{*} \otimes_{k G} Y_{*}\right) /\left(X_{*} \otimes_{k G} k_{0}\right)
$$

Corollary 66 There is spectral sequence converging (weakly) to $\widetilde{H} S_{*}(A)$ with

$$
E_{p, q}^{1} \cong \bigoplus_{u \in X^{p+1} / \Sigma_{p+1}} H_{p+q}\left(E_{*} G_{u} \bigotimes_{G_{u}} \operatorname{Sym}_{*}^{(p)} ; k\right),
$$

where $G_{u}$ is the isotropy subgroup for the chosen representative of $u \in X^{p+1} / \Sigma_{p+1}$.

\section{Properties of the complex $\operatorname{Sym}_{*}^{(p)}$}

\subsection{Algebra structure of $\mathrm{Sym}_{*}$}

We may consider $\operatorname{Sym}_{*} \stackrel{\text { def }}{=} \bigoplus_{p \geq 0} \operatorname{Sym}_{*}^{(p)}$ as a bigraded differential algebra, where $\operatorname{bideg}(W)=(p+1, i)$ for $W \in \operatorname{Sym}_{i}^{(p)}$. The product

$$
\otimes: \operatorname{Sym}_{i}^{(p)} \otimes \operatorname{Sym}_{j}^{(q)} \rightarrow \operatorname{Sym}_{i+j}^{(p+q+1)}
$$


is defined by $W \otimes V \stackrel{\text { def }}{=} W \otimes V^{\prime}$, where $V^{\prime}$ is obtained from $V$ by replacing each formal indeterminate $z_{r}$ by $z_{r+p+1}$ for $0 \leq r \leq q$. Equation (19) then implies

$$
d(W \otimes V)=d(W) \otimes V+(-1)^{\operatorname{bideg}(W)_{2}} W \otimes d(V),
$$

where $\operatorname{bideg}(W)_{2}$ is the second component of $\operatorname{bideg}(W)$.

Proposition 67 The product $\otimes$ is well-defined on the level of homology. Furthermore, this product (on both the chain level and homology level) is skew commutative in a twisted sense: $W \otimes V=(-1)^{i j} \tau(V \otimes W)$, where $\operatorname{bideg}(W)=(p+1, i), \operatorname{bideg}(V)=$ $(q+1, j)$, and $\tau$ is the permutation sending

$$
\begin{aligned}
&\{0,1, \ldots, q, q+1, q+2, \ldots, p+q, p+q+1\} \mapsto \\
&\{p+1, p+2, \ldots, p+q+1,0,1, \ldots, p-1, p\} .
\end{aligned}
$$

Proof Equation (21) implies the product passes to homology classes. Now, suppose $W=Y_{0} \otimes Y_{1} \otimes \cdots \otimes Y_{p-i} \in \operatorname{Sym}_{i}^{(p)}$ and $V=Z_{0} \otimes Z_{1} \otimes \cdots \otimes Z_{q-j} \in \operatorname{Sym}_{j}^{(p)}$.

$$
V \otimes W=V \otimes W^{\prime}=(-1)^{\alpha} W^{\prime} \otimes V,
$$

where $W^{\prime}$ is related to $W$ by replacing each $z_{r}$ by $z_{r+q+1}$. The exponent $\alpha=$ $\operatorname{deg}(V) \operatorname{deg}(W)=i j$ arises from the relations in $\operatorname{Sym}_{i+j}^{(p+q+1)}$. (The fact that $\operatorname{deg}(V)=$ $i$ and $\operatorname{deg}(W)=j$ may be made clear by observing that the degree of a formal tensor product in $\mathrm{Sym}_{*}^{(s)}$ is equal to the number of cut points, that is, the number of places where a tensor product symbol may be inserted.) Next, apply the block transformation $\tau$ to (22) to obtain $\tau(V \otimes W)=(-1)^{\alpha} \tau\left(W^{\prime} \otimes V\right)=(-1)^{\alpha} W \otimes V^{\prime}=(-1)^{\alpha} W \otimes V$, where $V^{\prime}$ is obtained by replacing $z_{r}$ by $z_{r+p+1}$ in $V$.

\subsection{Computer calculations}

In principle, the homology of $\operatorname{Sym}_{*}^{(p)}$ may be found by using a computer. In fact, we have the following results up to $p=7$ :

Theorem 68 For $0 \leq p \leq 7$, the groups $H_{*}\left(\operatorname{Sym}_{*}^{(p)}\right)$ are free abelian and have Poincaré polynomials $P_{p}(t) \stackrel{\text { def }}{=} P\left(H_{*}\left(\operatorname{Sym}_{*}^{(p)}\right) ; t\right)$ :

$$
\begin{array}{llrl}
P_{0}(t) & =1, & P_{1}(t) & =t, \\
P_{2}(t) & =t+2 t^{2}, & P_{3}(t) & =7 t^{2}+6 t^{3}, \\
P_{4}(t) & =43 t^{3}+24 t^{4}, & P_{5}(t) & =t^{3}+272 t^{4}+120 t^{5}, \\
P_{6}(t) & =36 t^{4}+1847 t^{5}+720 t^{6}, & P_{7}(t) & =829 t^{5}+13710 t^{6}+5040 t^{7} .
\end{array}
$$


Proof These computations were performed using scripts written for the computer algebra systems GAP [12] and Octave [7].

We conjecture that the $H_{*}\left(\operatorname{Sym}_{*}^{(p)}\right)$ is always free abelian.

\subsection{Representation theory of $H_{*}\left(\operatorname{Sym}_{*}^{(p)}\right)$}

By Remark 62, the groups $H_{i}\left(\operatorname{Sym}_{*}^{(p)} ; k\right)$ carry the structure of $k \Sigma_{p+1}$-modules, so it seems natural to investigate the irreducible representations comprising these modules.

Proposition 69 Let $C_{p+1} \hookrightarrow \Sigma_{p+1}$ be the cyclic group of order $p+1$, embedded into the symmetric group as the subgroup generated by the permutation

$$
\tau_{p} \stackrel{\text { def }}{=}(0, p, p-1, \ldots, 1) .
$$

Then there is a $\Sigma_{p+1}$-isomorphism $H_{p}\left(\operatorname{Sym}_{*}^{(p)}\right) \cong A C_{p+1} \uparrow \Sigma_{p+1}$, that is, the alternating representation of the cyclic group, induced up to the symmetric group. Note, for $p$ even, $A C_{p+1}$ coincides with the trivial representation $I C_{p+1}$.

Moreover, $H_{p}\left(\operatorname{Sym}_{*}^{(p)}\right)$ is generated by the elements $\sigma\left(b_{p}\right)$, for the distinct cosets $\sigma C_{p+1}$, where $b_{p} \stackrel{\text { def }}{=} \sum_{j=0}^{p}(-1)^{j p} \tau_{p}^{j}\left(z_{0} z_{1} \ldots z_{p}\right)$.

Proof Let $w$ be a general element of $\operatorname{Sym}_{p}^{(p)}, w=\sum_{\sigma \in \Sigma_{p+1}} c_{\sigma} \sigma\left(z_{0} z_{1} \ldots z_{p}\right)$, where $c_{\sigma}$ are constants in $k . H_{p}\left(\operatorname{Sym}_{*}^{(p)}\right)$ consists of those $w$ such that $d(w)=0$. That is,

$$
0=\sum_{\sigma \in \Sigma_{p+1}} \sum_{i=0}^{p-1}(-1)^{i} c_{\sigma} \sigma\left(z_{0} \ldots z_{i} \otimes z_{i+1} \ldots z_{p}\right) .
$$

Now for each $\sigma$, the terms corresponding to $\sigma\left(z_{0} \ldots z_{i} \otimes z_{i+1} \ldots z_{p}\right)$ occur in pairs in the above formula. The obvious term of the pair is $(-1)^{i} c_{\sigma} \sigma\left(z_{0} \ldots z_{i} \otimes z_{i+1} \ldots z_{p}\right)$. Not so obviously, the second term of the pair is

$$
(-1)^{(p-i-1) i}(-1)^{p-i-1} c_{\rho} \rho\left(z_{0} \ldots z_{p-i-1} \otimes z_{p-i} \ldots z_{p}\right),
$$

where $\rho=\sigma \tau_{p}^{p-i}$. Thus, if $d(w)=0$, then we must have

$$
(-1)^{i} c_{\sigma}+(-1)^{(p-i-1)(i+1)} c_{\rho}=0,
$$

or $c_{\rho}=(-1)^{(p-i)(i+1)} c_{\sigma}$. Set $j=p-i$, so that $c_{\rho}=(-1)^{j(p-j+1)} c_{\sigma}=(-1)^{j p} c_{\sigma}$. This shows that the only restrictions on the coefficients $c_{\sigma}$ are that the absolute values of coefficients corresponding to $\sigma, \sigma \tau_{p}, \sigma \tau_{p}^{2}, \ldots$ must be the same, and their corresponding signs in $w$ alternate if and only if $p$ is odd; otherwise, they have the same signs. 
Clearly, the elements $\sigma\left(b_{p}\right)$ for distinct cosets $\sigma C_{p+1}$ represents an independent set of generators over $k$ for $H_{p}\left(\operatorname{Sym}_{*}^{(p)}\right)$.

Observe that $b_{p}$ is invariant under the action of $\operatorname{sgn}\left(\tau_{p}\right) \tau_{p}$, and so $b_{p}$ generates an alternating representation $A C_{p+1}$ over $k$. Induced up to $\Sigma_{p+1}$, we obtain the representation $A C_{p+1} \uparrow \Sigma_{p+1}$ of dimension $(p+1) ! /(p+1)=p$ !, generated by the elements $\sigma\left(b_{p}\right)$ as in the proposition.

Definition 70 For a given proper partition $\lambda=\left[\lambda_{0}, \lambda_{1}, \lambda_{2}, \ldots, \lambda_{s}\right]$ of the $p+1$ integers $\{0,1, \ldots, p\}$, an element $W$ of $\operatorname{Sym}_{*}^{(p)}$ will designated as type $\lambda$ if it equivalent to $\pm\left(Y_{0} \otimes Y_{1} \otimes Y_{2} \otimes \cdots \otimes Y_{S}\right)$ with $\operatorname{deg}\left(Y_{i}\right)=\lambda_{i}-1$. That is, each $Y_{i}$ has $\lambda_{i}$ factors. The notation $\operatorname{Sym}_{\lambda}^{(p)}$ or $\operatorname{Sym}_{\lambda}$ will denote the $k$-submodule of $\operatorname{Sym}_{p-s}^{(p)}$ generated by all elements of type $\lambda$.

In what follows, $|\lambda|$ will refer to the number of components of $\lambda$. The action of $\Sigma_{p+1}$ leaves $\operatorname{Sym}_{\lambda}$ invariant for any given $\lambda$, so the there is a decomposition as $k \Sigma_{p+1}$-module:

$$
\operatorname{Sym}_{p-s}^{(p)}=\bigoplus_{\lambda \vdash(p+1),|\lambda|=s+1} \operatorname{Sym}_{\lambda} .
$$

Proposition 71 For a given proper partition $\lambda \vdash(p+1)$,

(a) $\operatorname{Sym}_{\lambda}$ contains exactly one alternating representation $A \Sigma_{p+1}$ iff $\lambda$ contains no repeated components.

(b) $\operatorname{Sym}_{\lambda}$ contains exactly one trivial representation $I \Sigma_{p+1}$ iff $\lambda$ contains no repeated even components.

Proof $\operatorname{Sym}_{\lambda}$ is a quotient of the regular representation, since it is the image of the $\Sigma_{p+1}-$ map $\pi_{\lambda}: k \Sigma_{p+1} \rightarrow \operatorname{Sym}_{\lambda}, \sigma \mapsto \psi_{\lambda} s$, where $s \in \Sigma_{p+1}^{\mathrm{op}}$ is the $\Delta S-$ automorphism of $[p]$ corresponding to $\sigma$ and $\psi_{\lambda}$ is a $\Delta$-morphism $[p] \rightarrow[|\lambda|]$ that sends the points $0, \ldots, \lambda_{0}-1$ to 0 , the points $\lambda_{0}, \ldots, \lambda_{0}+\lambda_{1}-1$ to 1 , and so on. Hence, there can be at most 1 copy of $A \Sigma_{p+1}$ and at most 1 copy of $I \Sigma_{p+1}$ in $\operatorname{Sym}_{\lambda}$.

Let $W$ be the "standard" element of $\operatorname{Sym}_{\lambda}$. That is, the indeterminates $z_{i}$ occur in $W$ in numerical order and the degrees of monomials of $W$ are in decreasing order. $A \Sigma_{p+1}$ exists in $\operatorname{Sym}_{\lambda}$ iff the element $V=\sum_{\sigma \in \Sigma_{p+1}} \operatorname{sgn}(\sigma) \sigma(W)$ is non-zero. Suppose that some component of $\lambda$ is repeated, say $\lambda_{i}=\lambda_{i+1}=\ell$. If $W=Y_{0} \otimes Y_{1} \otimes \cdots \otimes Y_{s}$, then $\operatorname{deg}\left(Y_{i}\right)=\operatorname{deg}\left(Y_{i+1}\right)=\ell-1$. Now, we know that $W=(-1)^{\operatorname{deg}\left(Y_{i}\right) \operatorname{deg}\left(Y_{i+1}\right)} Y_{0} \otimes \cdots \otimes$ $Y_{i+1} \otimes Y_{i} \otimes \cdots \otimes Y_{s}=-(-1)^{\ell} \alpha(W)$, for the permutation $\alpha \in \Sigma_{p+1}$ that exchanges the indices of indeterminates in $Y_{i}$ with those in $Y_{i+1}$ in an order-preserving way. 
In $V$, the term $\alpha(W)$ shows up with $\operatorname{sign} \operatorname{sgn}(\alpha)=(-1)^{\ell}$, thus cancelling with $W$. Hence, $V=0$, and no alternating representation exists.

If, on the other hand, no component of $\lambda$ is repeated, then no term $\pm \alpha(W)$ can be equivalent to $W$ for $\alpha \neq$ id, so $V$ survives as the generator of $A \Sigma_{p+1}$ in $\operatorname{Sym}_{\lambda}$.

A similar analysis applies for trivial representations. This time, we examine $U=$ $\sum_{\sigma \in \Sigma_{p+1}} \sigma(W)$, which would be a generator for $I \Sigma_{p+1}$ if it were non-zero. As before, if there is a repeated component, $\lambda_{i}=\lambda_{i+1}=\ell$, then $W=(-1)^{\ell-1} \alpha(W)$. However, this time, $W$ cancels with $\alpha(W)$ only if $\ell-1$ is odd. That is, $\left|\lambda_{i}\right|=\left|\lambda_{i+1}\right|$ is even. If $\ell-1$ is even, or if all $\lambda_{i}$ are distinct, then the element $U$ must be non-zero.

Proposition $72 H_{i}\left(\operatorname{Sym}_{*}^{(p)}\right)$ contains an alternating representation for each partition $\lambda \vdash(p+1)$ with $|\lambda|=p-i$ such that no component of $\lambda$ is repeated.

Proof This proposition will follow from the fact that $d(V)=0$ for any generator $V$ of an alternating representation in $\mathrm{Sym}_{\lambda}$. Then, by Schur's Lemma, the alternating representation must survive at the level of homology.

Let $V=\sum_{\sigma \in \Sigma_{p+1}} \operatorname{sgn}(\sigma) \sigma(W)$ be the generator mentioned in Proposition 71. $d(V)$ consists of individual terms $d_{j}(\sigma(W))=\sigma\left(d_{j}(W)\right)$ along with appropriate signs. For a given $j, d_{j}(W)$ is identical to $W$ except at some monomial $Y_{i}$, where a tensor product symbol is inserted. We will introduce some notation to make the argument a little cleaner. If $Y=z_{i_{0}} z_{i_{1}} \ldots z_{i_{r}}$ is a monomial, then the notation $Y\{s, \ldots, t\}$ refers to the monomial $z_{i_{s}} z_{i_{s+1}} \ldots z_{i_{t}}$, assuming $0 \leq s \leq t \leq r$. Now, we write

$$
d_{j}(W)=(-1)^{a+\ell} Y_{0} \otimes \cdots \otimes Y_{i}\{0, \ldots, \ell\} \otimes Y_{i}\{\ell+1, \ldots, m\} \otimes \cdots \otimes Y_{s},
$$

where $a=\operatorname{deg}\left(Y_{0}\right)+\cdots+\operatorname{deg}\left(Y_{i-1}\right)$. Use the relations in $\operatorname{Sym}_{*}$ to rewrite (24):

$$
(-1)^{(a+\ell)+\ell(m-\ell-1)} Y_{0} \otimes \cdots \otimes Y_{i}\{\ell+1, \ldots, m\} \otimes Y_{i}\{0, \ldots, \ell\} \otimes \cdots \otimes Y_{S}
$$

Let $\alpha$ be the permutation that relabels indices in such a way that $Y_{i}\{0, \ldots, m-\ell-1\} \mapsto$ $Y_{i}\{\ell+1, \ldots, m\}$ and $Y_{i}\{m-\ell, \ldots, m\} \mapsto Y_{i}\{0, \ldots, \ell\}$, so that the following is equivalent to $(25)$.

$$
(-1)^{a+m \ell-\ell^{2}} \alpha\left(Y_{0} \otimes \cdots \otimes Y_{i}\{0, \ldots, m-\ell-1\} \otimes Y_{i}\{m-\ell, \ldots, m\} \otimes \cdots \otimes Y_{S}\right)
$$

Now, (26) also occurs in $d_{j^{\prime}}(\operatorname{sgn}(\alpha) \alpha(W))$ for some $j^{\prime}$. This term looks like

$$
\begin{aligned}
\operatorname{sgn}(\alpha)(-1)^{a+m-\ell-1} \alpha\left(Y_{0} \otimes \cdots \otimes Y_{i}\{0, \ldots, m-\ell-1\}\right. & \\
& \left.\otimes Y_{i}\{m-\ell, \ldots, m\} \otimes \cdots \otimes Y_{s}\right)
\end{aligned}
$$


which equals

$$
(-1)^{m \ell-\ell^{2}+a-1} \alpha\left(Y_{0} \otimes \cdots \otimes Y_{i}\{0, \ldots, m-\ell-1\} \otimes Y_{i}\{m-\ell, \ldots, m\} \otimes \cdots \otimes Y_{S}\right)
$$

Comparing the signs of (28) and (26), we verify the two terms cancel each other out in the sum $d(V)$.

By Proposition 72, it is clear that if $p+1$ is a triangular number - that is, $p+1$ is of the form $r(r+1) / 2$ for some positive integer $r$, then the lowest dimension in which an alternating representation may occur is $p+1-r$, corresponding to the partition $\lambda=[r, r-1, \ldots, 2,1]$. A little algebra yields the following statement for any $p$.

Corollary $73 H_{i}\left(\operatorname{Sym}_{*}^{(p)}\right)$ contains an alternating representation in degree $p+1-r$, where $r=\lfloor\sqrt{2 p+9 / 4}-1 / 2\rfloor$. Moreover, there are no alternating representations present for $i \leq p-r$.

There is not much known about the other irreducible representations occurring in the homology groups of $\operatorname{Sym}_{*}^{(p)}$, however computational evidence shows that $H_{i}\left(\operatorname{Sym}_{*}^{(p)}\right)$ contains no trivial representation, $I \Sigma_{p+1}$, for $i \leq p-r$ ( $r$ as in the conjecture above) up to $p=50$.

\subsection{Connectivity of $\operatorname{Sym}_{*}^{(p)}$}

Quite recently, Vrećica and Živaljević [30] observed that the complex $\operatorname{Sym}_{*}^{(p)}$ is isomorphic to the suspension of the cycle-free chessboard complex $\Omega_{p+1}$ (in fact, the isomorphism takes the form $k\left[S \Omega_{p+1}^{+}\right] \rightarrow \operatorname{Sym}_{*}^{(p)}$, where $\Omega_{p+1}^{+}$is the augmented complex).

The $m$-chains of the complex $\Omega_{n}$ are generated by ordered lists,

$$
L=\left\{\left(i_{0}, j_{0}\right),\left(i_{1}, j_{1}\right), \ldots,\left(i_{m}, j_{m}\right)\right\}
$$

where $1 \leq i_{0}<i_{1}<\cdots<i_{m} \leq n$, all $1 \leq j_{s} \leq n$ are distinct integers, and the list $L$ is cycle-free. It may be easier to say what it means for $L$ not to be cycle free: $L$ is not cycle-free if there exists a subset $L_{c} \subseteq L$ and ordering of $L_{c}$ so that

$$
L_{c}=\left\{\left(\ell_{0}, \ell_{1}\right),\left(\ell_{1}, \ell_{2}\right), \ldots,\left(\ell_{t-1}, \ell_{t}\right),\left(\ell_{t}, \ell_{0}\right)\right\} .
$$

The face maps are defined by deltion of elements of $L$ :

$d_{s}\left(\left\{\left(i_{0}, j_{0}\right), \ldots,\left(i_{m}, j_{m}\right)\right\}\right) \stackrel{\text { def }}{=}\left\{\left(i_{0}, j_{0}\right), \ldots,\left(i_{s-1}, j_{s-1}\right),\left(i_{s+1}, j_{s+1}\right), \ldots,\left(i_{m}, j_{m}\right)\right\}$

For completeness, an explicit isomorphism shall be provided as part of the proof of the following proposition. 
Proposition 74 Let $\Omega_{n}^{+}$denote the augmented cycle-free $(n \times n)$-chessboard complex, where the unique $(-1)$-chain is represented by the empty $n \times n$ chessboard, and the boundary map on 0 -chains takes a vertex to the unique $(-1)$-chain. For each $p \geq 0$, there is a chain isomorphism, $\omega_{*}: k\left[S \Omega_{p+1}^{+}\right] \rightarrow \operatorname{Sym}_{*}^{(p)}$.

Proof Note that we may define the generating $m$-chains of $k\left[\Omega_{p+1}\right]$ as cycle-free lists $L$, with no requirement on the order of $L$, under the equivalence relation:

$$
\sigma . L \stackrel{\text { def }}{=}\left\{\left(i_{\sigma^{-1}(0)}, j_{\sigma^{-1}(0)}\right), \ldots,\left(i_{\sigma^{-1}(m)}, j_{\sigma^{-1}(m)}\right)\right\} \approx \operatorname{sgn}(\sigma) L,
$$

for $\sigma \in \Sigma_{m+1}$. Suppose $L$ is an $(m+1)$-chain of $S \Omega_{p+1}^{+}$(that is an $m$-chain of $\Omega_{p+1}^{+}$). Call a subset $L^{\prime} \subseteq L$ a queue if there is a reordering of $L^{\prime}$ such that

$$
L^{\prime}=\left\{\left(\ell_{0}, \ell_{1}\right),\left(\ell_{1}, \ell_{2}\right), \ldots,\left(\ell_{t-1}, \ell_{t}\right)\right\} .
$$

$L^{\prime}$ is called a maximal queue if it is not properly contained in any other queue. Since $L$ is supposed to be cycle-free, we can partition $L$ into some number of maximal queues, $L_{1}^{\prime}, L_{2}^{\prime}, \ldots, L_{q}^{\prime}$. Let $\sigma$ be a permutation representing the reordering of $L$ into maximal ordered queues.

Now, each maximal ordered queue $L_{i}^{\prime}$ will correspond to a monomial of formal indeterminates $z_{i}$ as follows.

$$
L_{s}^{\prime}=\left\{\left(\ell_{0}, \ell_{1}\right),\left(\ell_{1}, \ell_{2}\right), \ldots,\left(\ell_{t-1}, \ell_{t}\right)\right\} \mapsto z_{\ell_{0}-1} z_{\ell_{1}-1} \cdots z_{\ell_{t}-1} .
$$

For each maximal ordered queue, $L_{s}^{\prime}$, denote the monomial obtained by formula (29) by $Z_{s}$. Let $k_{1}, k_{2}, \ldots, k_{u}$ be the numbers in $\{0,1,2, \ldots, p\}$ such that $k_{r}+1$ does not appear in any pair $\left(i_{s}, j_{s}\right) \in L$. Now we may define $\omega_{*}$ on $L=L_{1}^{\prime} \cup L_{2}^{\prime} \cup \ldots \cup L_{q}^{\prime}$.

$$
\omega_{m+1}(L) \stackrel{\text { def }}{=} Z_{1} \otimes Z_{2} \otimes \cdots \otimes Z_{q} \otimes z_{k_{1}} \otimes z_{k_{2}} \otimes \cdots \otimes z_{k_{u}} .
$$

Observe, if $L=\varnothing$ is the $(-1)$-chain of $\Omega_{p+1}^{+}$, then there are no maximal queues in $L$, and so $\omega_{0}(\varnothing)=z_{0} \otimes z_{1} \otimes \cdots \otimes z_{p}$.

$\omega_{*}$ is a (well-defined) chain map with inverse given by essentially reversing the process. To each monomial $Z=z_{i_{0}} z_{i_{1}} \cdots z_{i_{t}}$ with $t>0$, there is an associated ordered queue $L^{\prime}=\left\{\left(i_{0}+1, i_{1}+1\right),\left(i_{1}+1, i_{2}+1\right), \ldots\left(i_{t-1}+1, i_{t}+1\right)\right\}$. If the monomial is a singleton, $Z=z_{i_{0}}$, the associated ordered queue will be the empty set. Now, given a generator $Z_{1} \otimes Z_{2} \otimes \cdots \otimes Z_{q} \in \operatorname{Sym}_{*}^{(p)}$, map it to the list $L \stackrel{\text { def }}{=} L_{1}^{\prime} \cup L_{2}^{\prime} \cup \ldots \cup L_{q}^{\prime}$, preserving the original order of indices.

Theorem $75 \operatorname{Sym}_{*}^{(p)}$ is $\left\lfloor\frac{2}{3}(p-1)\right\rfloor$-connected.

Proof See Vrećica and Živaljević [30, Theorem 10]. 
This remarkable fact yields the following useful corollaries:

Corollary 76 The spectral sequences of Theorem 57 and Corollary 66 converge strongly to $\tilde{H} S_{*}(A)$.

Proof The connectivity of the complexes $\operatorname{Sym}_{*}^{(p)}$ is a non-decreasing function of $p$. So for large enough $p$, we have $H_{n}\left(E_{*} G_{u} \otimes_{G_{u}} \operatorname{Sym}_{*}^{(p)}\right)=0$. For fixed $n$, the spectral sequence is guaranteed to collapse at a finite level.

Corollary 77 For each $i \geq 0$, there is a positive integer $N_{i}$ so that if $p \geq N_{i}$, there is an isomorphism $\tilde{H} S_{i}(A) \cong H_{i}\left(\mathscr{F}_{p} C_{*}\left(\right.\right.$ Epi $\left.\left.\Delta S, B_{*}^{\text {sym }} I\right)\right)$.

Corollary 78 If $A$ is finitely-generated over a Noetherian ground ring $k$, then $H S_{*}(A)$ is finitely-generated over $k$ in each degree.

The bounds on connectivity are conjectured to be tight. This is certainly true for $p \equiv 1(\bmod 3)$, based on Vrećica and Živaljević [30, Theorem 16]. [30, Corollary 12] establishes that either $H_{2 k}\left(\operatorname{Sym}_{*}^{(3 k-1)}\right) \neq 0$ or $H_{2 k}\left(\operatorname{Sym}_{*}^{(3 k)}\right) \neq 0$. For $k \leq 2$, both statements are true. When the latter condition is true, this gives a tight bound on connectivity for $p \equiv 0(\bmod 3)$. When the former is true, there is not enough information for a tight bound, since we are more interested in proving that $H_{2 k-1}\left(\mathrm{Sym}_{*}^{(3 k-1)}\right)$ is nonzero, since for $k=1,2$, we have computed the integral homology, $H_{1}\left(\operatorname{Sym}_{*}^{(2)}\right)=\mathbb{Z}$ and $H_{3}\left(\operatorname{Sym}_{*}^{(5)}\right)=\mathbb{Z}$.

\section{A partial resolution}

As before, $k$ is a commutative ground ring. In this section, we find an explicit partial resolution of the trivial $\Delta S^{\text {op }}$-module $\underline{k}$ by projective modules, allowing the computation of $H S_{0}(A)$ and $H S_{1}(A)$ for a unital associative $k$-algebra $A$. The resolution will be constructed through a number of technical lemmas.

For any small category $\mathscr{C}$ and object $X \in \operatorname{Obj}_{\mathfrak{C}}$, the $\mathscr{C}$-modules $k$ [Mor $\left.\mathscr{C}(X,-)\right)$ ] are projective (as $\mathscr{C}$-module), as are the $\mathscr{C}^{\text {op }}$-modules $k$ [Mor $\left.\operatorname{Mo}_{\mathscr{C}}(-, X)\right]$. In particular, each $k\left[\operatorname{Mor}_{\Delta S}(-,[q])\right]$ is a projective $\Delta S^{\text {op }}$-module. In proving exactness, it suffices to examine the individual sub- $k$-modules, $k\left[\operatorname{Mor}_{\Delta S}([n],[q])\right]$.

Lemma 79 For each $n \geq 0$, the sequence

$$
0 \longleftarrow k \stackrel{\epsilon}{\longleftarrow} k\left[\operatorname{Mor}_{\Delta S}([n],[0])\right] \stackrel{\rho}{\longleftarrow} k\left[\operatorname{Mor}_{\Delta S}([n],[2])\right]
$$


is exact, where $\epsilon$ is defined by $\epsilon(\phi)=1$ for any morphism $\phi:[n] \rightarrow[0]$, and $\rho$ is defined by $\rho(\psi)=\left(x_{0} x_{1} x_{2}\right) \circ \psi-\left(x_{2} x_{1} x_{0}\right) \circ \psi$ for any morphism $\psi:[n] \rightarrow[2]$ $\left(x_{0} x_{1} x_{2}\right.$ and $x_{2} x_{1} x_{0}$ are $\Delta S$ morphisms [2] $\rightarrow$ [0] written in tensor notation).

Proof Clearly, $\epsilon$ is surjective. Now, $\epsilon \rho=0$, since $\rho(\psi)$ consists of two morphisms with opposite signs. Let $\phi_{0}=x_{0} x_{1} \ldots x_{n}:[n] \rightarrow[0]$. The kernel of $\epsilon$ is spanned by elements $\phi-\phi_{0}$ for $\phi \in \operatorname{Mor}_{\Delta S}([n],[0])$. So, it suffices to show that the submodule of $k\left[\operatorname{Mor}_{\Delta S}([n],[0])\right]$ generated by $\left(x_{0} x_{1} x_{2}\right) \psi-\left(x_{2} x_{1} x_{0}\right) \psi$ for $\psi:[n] \rightarrow[2]$ contains all of the elements $\phi-\phi_{0}$. In other words, it suffices to find a sequence $\phi=\phi_{k}, \phi_{k-1}, \ldots, \phi_{2}, \phi_{1}, \phi_{0}$ so that each $\phi_{i}$ is obtained from $\phi_{i+1}$ by reversing the order of 3 (possibly emtpy) blocks, $X Y Z \rightarrow Z Y X$. Let $\phi=x_{i_{0}} x_{i_{1}} \ldots x_{i_{n}}$. If $\phi=\phi_{0}$, we may stop here. Otherwise, we may produce a sequence ending in $\phi_{0}$ by way of a certain family of rearrangements:

$k$-rearrangement $x_{i_{0}} x_{i_{1}} \ldots x_{i_{k-1}} x_{i_{k}} x_{k+1} \ldots x_{n} \rightsquigarrow x_{k+1} \ldots x_{n} x_{i_{k}} x_{i_{0}} x_{i_{1}} \ldots x_{i_{k-1}}$ such that $i_{k} \neq k$. That is, a $k$-rearrangement only applies to those monomials that agree with $\phi_{0}$ in the final $n-k$ indeterminates, but not in the final $n-k+1$ indeterminates. If $k=n$, then this rearrangement reduces to the cyclic rearrangment, $x_{i_{0}} x_{i_{1}} \ldots x_{i_{n}} \rightsquigarrow x_{i_{n}} x_{i_{0}} x_{i_{1}} \ldots x_{i_{n-1}}$.

Beginning with $\phi$, perform $n$-rearrangements until the final indeterminate is $x_{n}$. For convenience of notation, let this new monomial be $x_{j_{0}} x_{j_{1}} \ldots x_{j_{n}}$. (Of course, $j_{n}=n$.) If $j_{k}=k$ for all $k=0,1, \ldots, n$, then we are done. Otherwise, there will be a number $k$ such that $j_{k} \neq k$ but $j_{k+1}=k+1, \ldots, j_{n}=n$. Perform a $k$-rearrangement followed by enough $n$-rearrangements so that the final indeterminate is again $x_{n}$. The net result of these rearrangements is that the ending block $x_{k+1} x_{k+2} \ldots x_{n}$ remains fixed while the beginning block $x_{j_{0}} x_{j_{1}} \ldots x_{j_{k}}$ becomes cyclically permuted to $x_{j_{k}} x_{j_{0}} \ldots x_{j_{k-1}}$. It is clear that applying this combination of rearrangements repeatedly will finally obtain a monomial $x_{\ell_{0}} x_{\ell_{1}} \ldots x_{\ell_{k-1}} x_{k} x_{k+1} \ldots x_{n}$. Now repeat the process, until after a finite number of steps, we finally obtain $\phi_{0}$.

Let $\mathscr{B}_{n} \stackrel{\text { def }}{=}\left\{x_{i_{0}} x_{i_{1}} \ldots x_{i_{k-1}} \otimes x_{i_{k}} \otimes x_{k+1} x_{k+2} \ldots x_{n} \mid 1 \leq k \leq n, i_{k} \neq k\right\}$. Then $k\left[\mathscr{B}_{n}\right]$ is a free submodule of $k\left[\operatorname{Mor}_{\Delta S}([n],[2])\right]$ of size $(n+1) !-1$.

Corollary 80 When restricted to $k\left[\mathscr{B}_{n}\right]$, the map $\rho$ of Lemma 79 is surjective onto the kernel of $\epsilon$.

Proof In the proof of Lemma 79, the $n$-rearrangements correspond to the image of elements $x_{i_{0}} \ldots x_{i_{n-1}} \otimes x_{i_{n}} \otimes 1$, with $i_{n} \neq n$. For $k<n, k$-rearrangements correspond to the image of elements $x_{i_{0}} \ldots x_{i_{k-1}} \otimes x_{i_{k}} \otimes x_{k+1} \ldots x_{n}$, with $i_{k} \neq k$. 
Lemma $81 k\left[\operatorname{Mor}_{\Delta S}([n],[m])\right]$ is a free $k$-module of rank $(m+n+1) ! / m !$.

Proof A morphism $\phi:[n] \rightarrow[m]$ of $\Delta S$ is nothing more than an assignment of $n+1$ objects into $m+1$ compartments, along with a total ordering of the original $n+1$ objects, hence \# $\operatorname{Mor}_{\Delta S}([n],[m])=\left(\begin{array}{c}m+n+1 \\ m\end{array}\right)(n+1) !=\frac{(m+n+1) !}{m !}$.

Lemma $\left.82 \rho\right|_{k\left[\Re_{n}\right]}$ is an isomorphism $k\left[\mathscr{B}_{n}\right] \cong \operatorname{ker} \epsilon$.

Proof Since the rank of $k\left[\operatorname{Mor}_{\Delta S}([n],[0])\right]$ is $(n+1)$ !, the rank of the $k e r \epsilon$ is $(n+1) !-1$. The isomorphism then follows from Corollary 80 .

Lemma 83 The relations of the form

$$
X Y \otimes Z \otimes W+W \otimes Z X \otimes Y+Y Z X \otimes 1 \otimes W+W \otimes Y Z \otimes X \approx 0 \quad \text { and }
$$$$
1 \otimes X \otimes 1 \approx 0
$$

collapse $k\left[\operatorname{Mor}_{\Delta S}([n],[2])\right]$ onto $k\left[\Re_{n}\right]$.

Proof This proof proceeds in multiple steps.

Step 1: Degeneracy Relations $X \otimes Y \otimes 1 \approx X \otimes 1 \otimes Y \approx 1 \otimes X \otimes Y$.

First, observe that letting $X=Y=W=1$ in (31) yields $Z \otimes 1 \otimes 1 \approx 0$, since $1 \otimes Z \otimes 1 \approx 0$. Then, letting $X=Z=W=1$ in (31) produces $1 \otimes 1 \otimes Y \approx 0$. Thus, any formal tensor with two trivial factors is equivalent to 0 . Next, let $Z=W=1$ in (31). Then using the above observation, we obtain $1 \otimes X \otimes Y+1 \otimes Y \otimes X \approx 0$, that is, $1 \otimes X \otimes Y \approx-(1 \otimes Y \otimes X)$. Then, if we let $X=W=1$, we obtain $Y \otimes Z \otimes 1+1 \otimes Z \otimes Y \approx 0$, which is equivalent to $Y \otimes Z \otimes 1-1 \otimes Y \otimes Z \approx 0$. Finally, let $X=Y=1$ in (31). The expression reduces to $Z \otimes 1 \otimes W-1 \otimes Z \otimes W \approx 0$.

Step 2: Sign Relation $X \otimes Y \otimes Z \approx-(Z \otimes Y \otimes X)$.

Let $Y=1$ in (31), and use the degeneracy relations to rewrite the result as $X \otimes Z \otimes W+$ $1 \otimes W \otimes Z X+1 \otimes Z X \otimes W+W \otimes Z \otimes X \approx 0$. Since $1 \otimes Z X \otimes W \approx-(1 \otimes W \otimes Z W)$, the desired result follows: $X \otimes Z \otimes W+W \otimes Z \otimes X \approx 0$.

Step 3: Hochschild Relation $\quad X Y \otimes Z \otimes 1-X \otimes Y Z \otimes 1+Z X \otimes Y \otimes 1 \approx 0$. This relation is named after the similar relation, $X Y \otimes Z-X \otimes Y Z+Z X \otimes Y$, that arises in the Hochschild complex.

Let $W=1$ in (31), and use the degeneracy and sign relations to obtain the desired result. 
Step 4: Cyclic Relation $\sum_{j=0}^{n} \tau_{n}^{j}\left(x_{i_{0}} x_{i_{1}} \ldots x_{i_{n-1}} \otimes x_{i_{n}} \otimes 1\right) \approx 0$, where $\tau_{n} \in \Sigma_{n+1}$ is the $(n+1)$-cycle $(0, n, n-1, \ldots, 2,1)$, which acts by permuting the indices.

For $n=0$, there are no such relations (indeed, no relations at all). For $n=1$, the cyclic relation takes the form $x_{0} \otimes x_{1} \otimes 1+x_{1} \otimes x_{0} \otimes 1 \approx 0$, which follows from degeneracy and sign relations.

Assume now that $n \geq 2$. For each $k=1,2, \ldots, n-1$, define

$$
\begin{aligned}
& A_{k} \stackrel{\text { def }}{=} x_{i_{0}} x_{i_{1}} \ldots x_{i_{k-1}}, \\
& B_{k} \stackrel{\text { def }}{=} x_{i_{k}}, \\
& C_{k} \stackrel{\text { def }}{=} x_{i_{k+1}} \ldots x_{i_{n}} .
\end{aligned}
$$

By the Hochschild relation, $0 \approx \sum_{k=1}^{n-1}\left(A_{k} B_{k} \otimes C_{k} \otimes 1-A_{k} \otimes B_{k} C_{k} \otimes 1+C_{k} A_{k} \otimes\right.$ $\left.B_{k} \otimes 1\right)$. But for $k \leq n-2, A_{k} B_{k} \otimes C_{k} \otimes 1=A_{k+1} \otimes B_{k+1} C_{k+1} \otimes 1$. Thus, after some cancellation,

$$
0 \approx-A_{1} \otimes B_{1} C_{1} \otimes 1+A_{n-1} B_{n-1} \otimes C_{n-1} \otimes 1+\sum_{k=1}^{n-1} C_{k} A_{k} \otimes B_{k} \otimes 1 .
$$

Now observe that sign and degeneracy relations imply that $-A_{1} \otimes B_{1} C_{1} \otimes 1 \approx$ $x_{i_{1}} \ldots x_{i_{n}} \otimes x_{i_{0}} \otimes 1$. The term $A_{n-1} B_{n-1} \otimes C_{n-1} \otimes 1$ is equal to $x_{i_{0}} \ldots x_{i_{n-1}} \otimes x_{i_{n}} \otimes 1$, and for $1 \leq k \leq n-1, C_{k} A_{k} \otimes B_{k} \otimes 1=x_{i_{k+1}} \ldots x_{i_{n}} x_{i_{0}} \ldots x_{i_{k-1}} \otimes x_{i_{k}} \otimes 1$. Thus, (33) can be rewritten as the cyclic relation,

$$
0 \approx\left(x_{i_{0}} \ldots x_{i_{n-1}} \otimes x_{i_{n}} \otimes 1\right)+\sum_{k=0}^{n-1} x_{i_{k+1}} \ldots x_{i_{n}} x_{i_{0}} \ldots x_{i_{k-1}} \otimes x_{i_{k}} \otimes 1 .
$$

Step 5 Every element of the form $X \otimes Y \otimes 1$ is equivalent to a linear combination of elements of $\mathscr{P}_{n}$.

To prove this, we shall induct on the size of $Y$. Suppose $Y$ consists of a single indeterminate. That is, $X \otimes Y \otimes 1=x_{i_{0}} \ldots x_{i_{n-1}} \otimes x_{i_{n}} \otimes 1$. Now, if $i_{n} \neq n$, we are done. Otherwise, we use the cyclic relation to write $x_{i_{0}} \ldots x_{i_{n-1}} \otimes x_{i_{n}} \otimes 1 \approx$ $-\sum_{j=1}^{n} \tau_{n}^{j}\left(x_{i_{0}} \ldots x_{i_{n-1}} \otimes x_{i_{n}} \otimes 1\right)$.

Now suppose $k \geq 1$ and any element $Z \otimes W \otimes 1$ with $|W|=k$ is equivalent to an element of $k\left[\mathscr{B}_{n}\right]$. Consider $X \otimes Y \otimes 1=x_{i_{0}} \ldots x_{i_{n-k-1}} \otimes x_{i_{n-k}} \ldots x_{i_{n}} \otimes 1$. Let

$$
\begin{aligned}
& A=x_{i_{0}} x_{i_{1}} \ldots x_{i_{n-k-1}}, \\
& B=x_{i_{n-k}} \ldots x_{i_{n-1}}, \\
& C=x_{i_{n}} .
\end{aligned}
$$


Then, by the Hochschild relation, $X \otimes Y \otimes 1=A \otimes B C \otimes 1 \approx A B \otimes C \otimes 1+C A \otimes B \otimes 1$. But since $C$ has one indeterminate and $B$ has $k$ indeterminates, this last expression is equivalent to an element of $k\left[\mathscr{B}_{n}\right]$ by inductive hypothesis.

Step 6: Modified Hochschild Relation $\quad X Y \otimes Z \otimes W-X \otimes Y Z \otimes W+Z X \otimes$ $Y \otimes W \approx 0$, modulo $k\left[\mathscr{B}_{n}\right]$.

First, we show that $X \otimes Y \otimes W+Y \otimes X \otimes W \approx 0\left(\bmod k\left[\Re_{n}\right]\right)$. Indeed, if we let $Z=1$ in (31), then sign and degeneracy relations yield: $X \otimes Y \otimes W+Y \otimes X \otimes W \approx$ $X Y \otimes W \otimes 1+Y X \otimes W \otimes 1$, that is, by step 5,

$$
X \otimes Y \otimes W \approx-(Y \otimes X \otimes W) \quad\left(\bmod k\left[\mathscr{B}_{n}\right]\right) .
$$

Now, using sign and degeneracy relations, (31) can be re-expressed:

$$
X Y \otimes Z \otimes W-Y \otimes Z X \otimes W+Y Z X \otimes W \otimes 1-X \otimes Y Z \otimes W \approx 0 .
$$

Using (34), we then arrive at the modified Hochschild relation,

$$
X Y \otimes Z \otimes W-X \otimes Y Z \otimes W+Z X \otimes Y \otimes W \approx 0 \quad\left(\bmod k\left[\Re_{n}\right]\right) .
$$

Step 7 Modified Cyclic Relation $\sum_{j=0}^{k} \tau_{k}^{j}\left(x_{i_{0}} x_{i_{1}} \ldots x_{i_{k-1}} \otimes x_{i_{k}} \otimes x_{i_{k+1}} \ldots x_{i_{n}}\right) \approx$ 0 , modulo $k\left[\mathscr{B}_{n}\right]$. Note, the $(k+1)$-cycle $\tau_{k}$ permutes the indices $i_{0}, i_{1}, \ldots, i_{k}$, and fixes the rest.

Equation (34) proves the modified cyclic relations for $k=1$. The modified cyclic relation for $k \geq 2$ follows from the modified Hochschild relation in the same manner as in step 4. Of course, this time all equivalences are taken modulo $k\left[\mathscr{R}_{n}\right]$.

Step 8 Every element of the form $X \otimes Y \otimes x_{n}$ is equivalent to an element of $k\left[\mathscr{S}_{n}\right]$.

We shall use the modified cyclic and modified Hochschild relations in a similar way as cyclic and Hochschild relations were used in step 5. Again we induct on the size of $Y$. If $|Y|=1$, then $X \otimes Y \otimes x_{n}=x_{i_{0}} \ldots x_{i_{n-2}} \otimes x_{i_{n-1}} \otimes x_{n}$. If $i_{n-1} \neq n-1$, then we are done. Otherwise, use the modified cyclic relation to re-express $X \otimes Y \otimes x_{n}$ as a sum of elements of $k\left[\Re_{n}\right]$.

Next, suppose $k \geq 1$ and any element $Z \otimes W \otimes x_{n}$ with $|W|=k$ is equivalent to an element of $k\left[\mathscr{\Re}_{n}\right]$. Consider an element $X \otimes Y \otimes x_{n}$ with $|Y|=k+1$. Write $Y=B C$ with $|B|=k$ and $|C|=1$ and use the modified Hochschild relation to rewrite $X \otimes B C \otimes x_{n}$ in terms of two elements whose middle tensor factors are either $B$ or $C$ (modulo $k\left[\mathscr{P}_{n}\right]$ ). By inductive hypothesis, the rewritten expression must lie in $k\left[\mathscr{B}_{n}\right]$. 
Step 9 Every element of $k\left[\operatorname{Mor}_{\Delta S}([n],[2])\right]$ is equivalent to a linear combination of elements from the following set:

$$
\begin{aligned}
& \mathscr{C}_{n} \stackrel{\text { def }}{=}\left\{X \otimes x_{i_{n}} \otimes 1 \mid i_{n} \neq n\right\} \cup\left\{X \otimes x_{i_{n-1}} \otimes x_{n} \mid\right.\left.i_{n-1} \neq n-1\right\} \\
& \cup\left\{X \otimes Y \otimes Z x_{n}|| Z \mid \geq 1\right\}
\end{aligned}
$$

Note, the $k$-module generated by $\mathscr{C}_{n}$ contains $k\left[\mathscr{B}_{n}\right]$.

Let $X \otimes Y \otimes Z$ be an arbitrary element of $k\left[\operatorname{Mor}_{\Delta S}([n],[2])\right]$. If $|X|=0,|Y|=0$, or $|Z|=0$, then the degeneracy relations and step 5 imply that $X \otimes Y \otimes Z$ is equivalent to an element of $k\left[\mathscr{B}_{n}\right]$.

Suppose now that $|X|,|Y|,|Z| \geq 1$. If $x_{n}$ occurs in $X$, use the relation $X \otimes Y \otimes W \approx$ $-(Y \otimes X \otimes W)\left(\bmod k\left[\mathscr{B}_{n}\right]\right)$ to ensure that $x_{n}$ occurs in the middle factor. If $x_{n}$ occurs in $Z$, use the sign relation and the above relation to put $x_{n}$ into the middle factor. In any case, it suffices to assume our element has the form: $X \otimes U x_{n} V \otimes Z$. Using the modified Hochschild relation, $X \otimes U x_{n} V \otimes Z \approx-\left(Z \otimes V \otimes X U x_{n}\right)+Z \otimes V X \otimes U x_{n}$, $\left(\bmod k\left[\mathscr{S}_{n}\right]\right)$. The first term is certainly in $k\left[\mathscr{C}_{n}\right]$, since $|X| \geq 1$. If $|U|>0$, the second term also lies in $k\left[\mathscr{C}_{n}\right]$. If, on the other hand, $|U|=0$, then step 8 implies that $Z \otimes V X \otimes x_{n}$ is an element of $k\left[\mathscr{B}_{n}\right]$.

Observe that Step 9 proves Lemma 83 for $n=0,1,2$, since in these cases, any elements that fall within the set $\left\{X \otimes Y \otimes Z x_{n}|| Z \mid \geq 1\right\}$ must have either $|X|=0$ or $|Y|=0$, hence are equivalent via the degeneracy relation to elements of $k\left[\left\{X \otimes x_{i_{n}} \otimes 1 \mid i_{n} \neq n\right\}\right]$. In what follows, assume $n \geq 3$.

Step 10 Every element of $k\left[\operatorname{Mor}_{\Delta S}([n],[2])\right]$ is equivalent, modulo $k\left[\mathscr{S}_{n}\right]$, to a linear combination of elements from the following set:

$$
\mathscr{D}_{n} \stackrel{\text { def }}{=}\left\{X \otimes x_{i_{n-2}} \otimes x_{n-1} x_{n} \mid i_{n-2} \neq n-2\right\} \cup\left\{X \otimes Y \otimes Z x_{n-1} x_{n}|| Z \mid \geq 1\right\} .
$$

First, we require a relation that transports $x_{n}$ from the end of a tensor:

$$
W \otimes Z \otimes X x_{n} \approx W \otimes x_{n} Z \otimes X \quad\left(\bmod k\left[\mathscr{S}_{n}\right]\right)
$$

Letting $Y=x_{n}$ in (31), and making use of the sign relation, we have: $W \otimes Z \otimes$ $X x_{n} \approx W \otimes Z X \otimes x_{n}+x_{n} Z X \otimes W \otimes 1+W \otimes x_{n} Z \otimes X$. By steps 5 and 8 , $W \otimes Z \otimes X x_{n} \approx W \otimes x_{n} Z \otimes X$, modulo elements of $k\left[\mathscr{B}_{n}\right]$.

Now, let $X \otimes Y \otimes Z$ be an arbitrary element of $k\left[\operatorname{Mor}_{\Delta S}([n],[2])\right]$. Locate $x_{n-1}$ and use the techniques of Step 9 to re-express $X \otimes Y \otimes Z$ as a linear combination of terms of the form: $X_{j} \otimes Y_{j} \otimes Z_{j} x_{n-1}$, modulo $k\left[\mathscr{B}_{n}\right]$. Our goal is to re-express each term as a linear combination of vectors in which $x_{n}$ occurs only in the second tensor factor. 
If $x_{n}$ occurs in $X_{j}$, then observe $X_{j} \otimes Y_{j} \otimes Z_{j} x_{n-1} \approx-\left(Y_{j} \otimes X_{j} \otimes Z_{j} x_{n-1}\right)$, $\left(\bmod k\left[\Re_{n}\right]\right)$.

If $x_{n}$ occurs in $Z_{j}$, then first substitute $Y=x_{n-1}$ into (31), obtaining the relation:

$$
\begin{array}{r}
X x_{n-1} \otimes Z \otimes W+W \otimes Z X \otimes x_{n-1}+x_{n-1} Z X \otimes 1 \otimes W+W \otimes x_{n-1} Z \otimes X \approx 0 \\
\Rightarrow W \otimes Z \otimes X x_{n-1} \approx W \otimes Z X \otimes x_{n-1}+W \otimes x_{n-1} Z \otimes X \quad\left(\bmod k\left[\Re_{n}\right]\right)
\end{array}
$$

By the modified Hochschild relation, $W \otimes x_{n-1} Z \otimes X \approx W x_{n-1} \otimes Z \otimes X+Z W \otimes$ $x_{n-1} \otimes X, \quad\left(\bmod k\left[\mathscr{P}_{n}\right]\right)$, then using sign relations etc., we obtain:

$W \otimes Z \otimes X x_{n-1} \approx$

$W \otimes Z X \otimes x_{n-1}+Z \otimes X \otimes W x_{n-1}-Z W \otimes X \otimes x_{n-1}, \quad\left(\bmod k\left[\Re_{n}\right]\right)$.

Thus, we can express our original element $X \otimes Y \otimes Z$ as a linear combination of elements of the form $X^{\prime} \otimes U^{\prime} x_{n} V^{\prime} \otimes Z^{\prime} x_{n-1}, \quad\left(\bmod k\left[\mathscr{B}_{n}\right]\right)$. Then using modified Hochschild etc., rewrite each such term as follows:

$X^{\prime} \otimes U^{\prime} x_{n} V^{\prime} \otimes Z^{\prime} x_{n-1} \approx$

$$
X^{\prime} U^{\prime} \otimes x_{n} V^{\prime} \otimes Z^{\prime} x_{n-1}-U^{\prime} \otimes x_{n} V^{\prime} X^{\prime} \otimes Z^{\prime} x_{n-1}, \quad\left(\bmod k\left[\Re_{n}\right]\right) .
$$

By (38), we transport $x_{n}$ to the end of each term, so $X^{\prime} \otimes U^{\prime} x_{n} V^{\prime} \otimes Z^{\prime} x_{n-1} \approx$ $X^{\prime} U^{\prime} \otimes V^{\prime} \otimes Z^{\prime} x_{n-1} x_{n}-U^{\prime} \otimes V^{\prime} X^{\prime} \otimes Z^{\prime} x_{n-1} x_{n}$, modulo elements of $k\left[\Re_{n}\right]$. If $\left|Z^{\prime}\right| \geq 1$, then we are done. Otherwise, we have some elements of the form $X^{\prime \prime} \otimes Y^{\prime \prime} \otimes x_{n-1} x_{n}$. Use an induction argument analogous to that in step 8 to re-express this type of element as a linear combination of elements of the form $U \otimes x_{i_{n-2}} \otimes x_{n-1} x_{n}$ such that $i_{n-2} \neq n-2$, modulo elements of $k\left[\Re_{n}\right]$.

Step 11 Every element of $k\left[\operatorname{Mor}_{\Delta S}([n],[2])\right]$ is equivalent to an element of $k\left[\Re_{n}\right]$.

We shall use an iterative re-writing procedure. First of all, define sets:

$$
\begin{aligned}
& \mathscr{B}_{n}^{j} \stackrel{\text { def }}{=}\left\{A \otimes x_{i_{n-j}} \otimes x_{n-j+1} \ldots x_{n} \mid i_{n-j} \neq n-j\right\}, \\
& \mathscr{C}_{n}^{j} \stackrel{\text { def }}{=}\left\{A \otimes B \otimes C x_{n-j+1} \ldots x_{n}|| C \mid \geq 1\right\} .
\end{aligned}
$$

Now clearly, $\mathscr{B}_{n}=\bigcup_{j=0}^{n-1} \mathscr{B}_{n}^{j}$. In what follows, 'reduced' will always mean reduced modulo elements of $k\left[\mathscr{P}_{n}\right]$. By steps 9 and 10, we can reduce an arbitrary element $X \otimes Y \otimes Z$ to linear combinations of elements in $\mathscr{B}_{n}^{0} \cup \mathscr{B}_{n}^{1} \cup \mathscr{B}_{n}^{2} \cup \mathscr{C}_{n}^{2}$. Suppose now that we have reduced elements to linear combinations of elements from the set $\mathscr{B}_{n}^{0} \cup \mathscr{B}_{n}^{1} \cup \ldots \cup \mathscr{B}_{n}^{j} \cup \mathscr{C}_{n}^{j}$, for some $j \geq 2$. I claim any element of $\mathscr{C}_{n}^{j}$ can be re-expressed as a linear combination of elements from the set $\mathscr{B}_{n}^{0} \cup \mathscr{B}_{n}^{1} \cup \ldots \cup \mathscr{B}_{n}^{j+1} \cup \mathscr{C}_{n}^{j+1}$. Indeed, let $X \otimes Y \otimes Z x_{n-j+1} \ldots x_{n}$, with $|Z| \geq 1$. Let $w \stackrel{\text { def }}{=} x_{n-j+1} \ldots x_{n}$. We may now 
think of $X \otimes Y \otimes Z w$ as consisting of the 'indeterminates' $x_{0}, x_{1}, \ldots, x_{n-j}, w$, hence, by step 10 , we may reduce this element to a linear combination of elements from the set $\left\{X \otimes x_{i_{n-j-1}} \otimes x_{n-j} w \mid i_{n-j-1} \neq n-j-1\right\} \cup\left\{X \otimes Y \otimes Z x_{n-j} w|| Z \mid \geq 1\right\}$. This implies the element may written as a linear combination of elements from the set $\mathscr{B}_{n}^{j+1} \cup \mathscr{C}_{n}^{j+1}$, modulo elements of the form $A \otimes B \otimes 1$ and $A \otimes B \otimes x_{n-j+1} \ldots x_{n}$. Since $\left\{A \otimes B \otimes x_{n-j+1} x_{n-j+2} \ldots x_{n}\right\} \subseteq \mathscr{C}_{n}^{j-1}$, the inductive hypothesis ensures that the there is set containment $\left\{A \otimes B \otimes x_{n-j+1} \ldots x_{n}\right\} \subseteq \mathscr{B}_{n}^{0} \cup \ldots \cup \mathscr{P}_{n}^{j}$. This completes the inductive step.

After a finite number of iterations, then, we can re-express any element $X \otimes Y \otimes Z$ as a linear combination from the set $\mathscr{B}_{n}^{0} \cup \ldots \cup \mathscr{B}_{n}^{n-1} \cup \mathscr{C}_{n}^{n-1}=\mathscr{B}_{n} \cup \mathscr{C}_{n}^{n-1}$. But $\mathscr{C}_{n}^{n-1}=\left\{A \otimes B \otimes C x_{2} \ldots x_{n}|| C \mid \geq 1\right\}$. Any element from this set has either $|A|=0$ or $|B|=0$, therefore is equivalent to an element of $k\left[\mathscr{B}_{n}\right]$ already.

Corollary 84 If $\frac{1}{2} \in k$, then the four-term relation $X Y \otimes Z \otimes W+W \otimes Z X \otimes Y+$ $Y Z X \otimes 1 \otimes W+W \otimes Y Z \otimes X \approx 0$ is sufficient to collapse $k\left[\operatorname{Mor}_{\Delta S}([n],[2])\right]$ onto $k\left[\mathscr{B}_{n}\right]$.

Proof We only need to modify step 1 of the previous proof. We will establish that $X \otimes 1 \otimes 1 \approx 1 \otimes X \otimes 1 \approx 1 \otimes 1 \otimes X \approx 0$.

Setting three variables at a time equal to 1 in (31) we obtain,

$$
\begin{aligned}
2(W \otimes 1 \otimes 1)+2(1 \otimes 1 \otimes W) \approx 0, & \text { when } X=Y=Z=1 . \\
Z \otimes 1 \otimes 1+3(1 \otimes Z \otimes 1) \approx 0, & \text { when } X=Y=W=1 . \\
2(Y \otimes 1 \otimes 1)+1 \otimes Y \otimes 1+1 \otimes 1 \otimes Y \approx 0, & \text { when } X=Z=W=1 .
\end{aligned}
$$

Equivalently, we have a system of linear equations,

$$
\left[\begin{array}{lll}
2 & 0 & 2 \\
1 & 3 & 0 \\
2 & 1 & 1
\end{array}\right]\left[\begin{array}{l}
z_{1} \\
z_{2} \\
z_{3}
\end{array}\right]=0,
$$

where $z_{1}=X \otimes 1 \otimes 1, z_{2}=1 \otimes X \otimes 1$, and $z_{3}=1 \otimes 1 \otimes X$. Since the determinant of the coefficient matrix is -4 , the matrix is invertible in the ring $k$ as long as $1 / 2 \in k$.

Define for each $m \geq 0, P_{m} \stackrel{\text { def }}{=} k\left[\operatorname{Mor}_{\Delta S}(-,[m])\right]$ and $P_{m}^{n}=P_{m}([n])$. Lemma 83 together with Lemmas 82 and 79 show the following sequence of $k$-modules is exact for each $n \geq 0$ :

$$
0 \longleftarrow k \stackrel{\epsilon}{\longleftarrow} P_{0}^{n} \stackrel{\rho}{\longleftarrow} P_{2}^{n} \stackrel{(\alpha, \beta)}{\longleftarrow} P_{3}^{n} \oplus P_{0}^{n}
$$


where $\alpha: P_{3}^{n} \rightarrow P_{2}^{n}$ is given by composition with the $\Delta S$ morphism

$$
x_{0} x_{1} \otimes x_{2} \otimes x_{3}+x_{3} \otimes x_{2} x_{0} \otimes x_{1}+x_{1} x_{2} x_{0} \otimes 1 \otimes x_{3}+x_{3} \otimes x_{1} x_{2} \otimes x_{0},
$$

and $\beta: P_{0}^{n} \rightarrow P_{2}^{n}$ is induced by $1 \otimes x_{0} \otimes 1$. This holds for all $n \geq 0$, so we have constructed a partial resolution of $\underline{k}$ by projective $\Delta S^{\text {op }}$-modules:

$$
0 \longleftarrow k \stackrel{\epsilon}{\longleftarrow} P_{0} \stackrel{\rho}{\longleftarrow} P_{2} \stackrel{(\alpha, \beta)}{\longleftarrow} P_{3} \oplus P_{0}
$$

\section{Using the partial resolution for low degree computations}

Let $A$ be a unital associative algebra over $k$. Since (43) is a partial resolution of $\underline{k}$, it can be used to find $H S_{i}(A)$ for $i=0,1$.

\subsection{Main theorem}

Theorem $85 H S_{i}(A)$ for $i=0,1$ may be computed as the degree 0 and degree 1 homology groups of the following (partial) chain complex:

$$
0 \longleftarrow A \stackrel{\partial_{1}}{\longleftarrow} A \otimes A \otimes A \stackrel{\partial_{2}}{\longleftarrow}(A \otimes A \otimes A \otimes A) \oplus A,
$$

where

$$
\begin{aligned}
& \partial_{1}: a \otimes b \otimes c \mapsto a b c-c b a, \\
& \partial_{2}: \begin{cases}a \otimes b \otimes c \otimes d & \mapsto a b \otimes c \otimes d+d \otimes c a \otimes b+b c a \otimes 1 \otimes d+d \otimes b c \otimes a, \\
a & \mapsto 1 \otimes a \otimes 1 .\end{cases}
\end{aligned}
$$

Proof Tensoring the complex (43) with $B_{*}^{\text {sym }} A$ over $\Delta S$, we obtain a complex that computes $H S_{0}(A)$ and $H S_{1}(A)$. The statement of the theorem then follows from isomorphisms induced by the evaluation map

$$
k\left[\operatorname{Mor}_{\Delta S}(-,[p])\right] \otimes_{\Delta S} B_{*}^{\mathrm{sym}} A \stackrel{\cong}{\longrightarrow} B_{p}^{\mathrm{sym}} A .
$$

\subsection{Degree 0 symmetric homology}

Theorem 86 For a unital associative algebra $A$ over commutative ground ring $k$, $H S_{0}(A) \cong A /([A, A])$, where $([A, A])$ is the ideal generated by the commutator submodule $[A, A]$.

Proof By Theorem $5, H S_{0}(A) \cong A / k[\{a b c-c b a\}]$ as a $k$-module. But $k[\{a b c-$ $c b a\}]$ is an ideal of $A$. Now clearly $[A, A] \subseteq k[\{a b c-b c a\}]$. On the other hand, $k[\{a b c-c b a\}] \subseteq([A, A])$ since $a b c-c b a=a(b c-c b)+a(c b)-(c b) a$. 
Corollary 87 If $A$ is commutative, then $H S_{0}(A) \cong A$.

Remark 88 Theorem 86 implies that symmetric homology does not preserve Morita equivalence, since for $n>1, H S_{0}\left(M_{n}(A)\right)=M_{n}(A) /\left(\left[M_{n}(A), M_{n}(A)\right]\right)=0$, while in general $H S_{0}(A)=A /([A, A]) \neq 0$.

\subsection{Degree 1 symmetric homology}

Using GAP, we have made the following explicit computations of degree 1 integral symmetric homology. See Section 13 for a discussion of how computer algebra systems were used in symmetric homology computations.

\begin{tabular}{l|l}
$A$ & $H S_{1}(A \mid \mathbb{Z})$ \\
\hline $\mathbb{Z}[t] /\left(t^{2}\right)$ & $\mathbb{Z} / 2 \mathbb{Z} \oplus \mathbb{Z} / 2 \mathbb{Z}$ \\
$\mathbb{Z}[t] /\left(t^{3}\right)$ & $\mathbb{Z} / 2 \mathbb{Z} \oplus \mathbb{Z} / 2 \mathbb{Z}$ \\
$\mathbb{Z}[t] /\left(t^{4}\right)$ & $(\mathbb{Z} / 2 \mathbb{Z})^{4}$ \\
$\mathbb{Z}[t] /\left(t^{5}\right)$ & $(\mathbb{Z} / 2 \mathbb{Z})^{4}$ \\
$\mathbb{Z}[t] /\left(t^{6}\right)$ & $(\mathbb{Z} / 2 \mathbb{Z})^{6}$ \\
\hline $\mathbb{Z}\left[C_{2}\right]$ & $\mathbb{Z} / 2 \mathbb{Z} \oplus \mathbb{Z} / 2 \mathbb{Z}$ \\
$\mathbb{Z}\left[C_{3}\right]$ & 0 \\
$\mathbb{Z}\left[C_{4}\right]$ & $(\mathbb{Z} / 2 \mathbb{Z})^{4}$ \\
$\mathbb{Z}\left[C_{5}\right]$ & 0 \\
$\mathbb{Z}\left[C_{6}\right]$ & $(\mathbb{Z} / 2 \mathbb{Z})^{6}$ \\
\hline
\end{tabular}

Based on these calculations, we conjecture:

\section{Conjecture 89}

$$
H S_{1}\left(k[t] /\left(t^{n}\right)\right)= \begin{cases}(k / 2 k)^{n}, & \text { if } n \geq 0 \text { is even. } \\ (k / 2 k)^{n-1} & \text { if } n \geq 1 \text { is odd. }\end{cases}
$$

Remark 90 The computations of $H S_{1}\left(\mathbb{Z}\left[C_{n}\right]\right)$ are consistent with those of Brown and Loday [2]. See Section 11.5 for a more detailed treatment of $H S_{1}$ for group rings.

Additionally, $H S_{1}$ has been computed for the following examples. These computations were done using GAP in some cases and in others, Fermat [15] computations on sparse matrices were used in conjunction with the GAP scripts. (for example, when the algebra has dimension greater than 6 over $\mathbb{Z}$ ). 


\begin{tabular}{l|l}
$A$ & $H S_{1}(A \mid \mathbb{Z})$ \\
\hline $\mathbb{Z}[t, u] /\left(t^{2}, u^{2}\right)$ & $\mathbb{Z} \oplus(\mathbb{Z} / 2 \mathbb{Z})^{11}$ \\
$\mathbb{Z}[t, u] /\left(t^{3}, u^{2}\right)$ & $\mathbb{Z}^{2} \oplus(\mathbb{Z} / 2 \mathbb{Z})^{11} \oplus \mathbb{Z} / 6 \mathbb{Z}$ \\
$\mathbb{Z}[t, u] /\left(t^{3}, u^{2}, t^{2} u\right)$ & $\mathbb{Z}^{2} \oplus(\mathbb{Z} / 2 \mathbb{Z})^{10}$ \\
$\mathbb{Z}[t, u] /\left(t^{3}, u^{3}\right)$ & $\mathbb{Z}^{4} \oplus(\mathbb{Z} / 2 \mathbb{Z})^{7} \oplus(\mathbb{Z} / 6 \mathbb{Z})^{5}$ \\
$\mathbb{Z}[t, u] /\left(t^{2}, u^{4}\right)$ & $\mathbb{Z}^{3} \oplus(\mathbb{Z} / 2 \mathbb{Z})^{20} \oplus \mathbb{Z} / 4 \mathbb{Z}$ \\
$\mathbb{Z}[t, u, v] /\left(t^{2}, u^{2}, v^{2}\right)$ & $\mathbb{Z}^{6} \oplus(\mathbb{Z} / 2 \mathbb{Z})^{42}$ \\
$\mathbb{Z}[t, u] /\left(t^{4}, u^{3}\right)$ & $\mathbb{Z}^{6} \oplus(\mathbb{Z} / 2 \mathbb{Z})^{19} \oplus \mathbb{Z} / 6 \mathbb{Z} \oplus(\mathbb{Z} / 12 \mathbb{Z})^{2}$ \\
$\mathbb{Z}[t, u, v] /\left(t^{2}, u^{2}, v^{3}\right)$ & $\mathbb{Z}^{11} \oplus(\mathbb{Z} / 2 \mathbb{Z})^{45} \oplus(\mathbb{Z} / 6 \mathbb{Z})^{4}$ \\
$\mathbb{Z}[i, j, k], i^{2}=j^{2}=k^{2}=i j k=-1$ & $(\mathbb{Z} / 2 \mathbb{Z})^{8}$ \\
$\mathbb{Z}\left[C_{2} \times C_{2}\right]$ & $(\mathbb{Z} / 2 \mathbb{Z})^{12}$ \\
$\mathbb{Z}\left[C_{3} \times C_{2}\right]$ & $(\mathbb{Z} / 2 \mathbb{Z})^{6}$ \\
$\mathbb{Z}\left[C_{3} \times C_{3}\right]$ & $(\mathbb{Z} / 3 \mathbb{Z})^{9}$ \\
$\mathbb{Z}\left[S_{3}\right]$ & $(\mathbb{Z} / 2 \mathbb{Z})^{2}$ \\
\hline
\end{tabular}

\subsection{Splittings of the partial complex}

Under certain circumstances, the partial complex in Theorem 5 splits as a direct sum of smaller complexes. This observation becomes increasingly important as the dimension of the algebra increases. Indeed, some of the computations of the previous section were done using splittings.

Definition 91 For a commutative $k$-algebra $A$ and $u \in A$, define the $k$-modules:

$$
\left(A^{\otimes n}\right)_{u} \stackrel{\text { def }}{=}\left\{a_{1} \otimes a_{2} \otimes \cdots \otimes a_{n} \in A^{\otimes n} \mid a_{1} a_{2} \ldots a_{n}=u\right\}
$$

Proposition 92 If $A=k[M]$ for a commutative monoid $M$, then complex (44) splits as a direct sum of complexes

$$
0 \longleftarrow(A)_{u} \stackrel{\partial_{1}}{\longleftarrow}(A \otimes A \otimes A)_{u} \stackrel{\partial_{2}}{\longleftarrow}(A \otimes A \otimes A \otimes A)_{u} \oplus(A)_{u},
$$

where $u$ ranges over the elements of $M$. Thus, for $i=0,1$, we have $H S_{i}(A) \cong$ $\bigoplus_{u \in M} H S_{i}(A)_{u}$.

Proof Since $M$ is a commutative monoid, there are direct sum decompositions as $k-$ module: $A^{\otimes n}=\bigoplus_{u \in M}\left(A^{\otimes n}\right)_{u}$. The boundary maps $\partial_{1}$ and $\partial_{2}$ preserve the products of tensor factors, so the inclusions $\left(A^{\otimes n}\right)_{u} \hookrightarrow A^{\otimes n}$ induce maps of complexes, hence the complex itself splits as a direct sum.

Definition 93 For each $u$, the homology groups of complex (45) will be called the $u$-layered symmetric homology of $A$, denoted $H S_{i}(A)_{u}$. 
We may use layers to investigate the symmetric homology of $k[t]$. This algebra is monoidal, generated by the monoid $\left\{1, t, t^{2}, t^{3}, \ldots\right\}$. Now, the $t^{m}$-layer symmetric homology of $k[t]$ will be the same as the $t^{m}$-layer symmetric homology of $k\left[M_{m+1}^{m+2}\right]$, where $M_{q}^{p}$ denotes the cyclic monoid generated by an indeterminate $s$ with the property that $s^{p}=s^{q}$. Using this observation and subsequent computation, we conjecture:

\section{Conjecture 94}

$$
H S_{1}(k[t])_{t^{m}}= \begin{cases}0 & m=0,1 \\ k / 2 k, & m \geq 2\end{cases}
$$

This conjecture has been verified up to $m=18$, in the case $k=\mathbb{Z}$.

\subsection{2-torsion in $H S_{1}$}

The occurrence of 2-torsion in $H S_{1}(A)$ for the examples considered in Sections 11.3 and 11.4 comes as no surprise, based on Theorem 2. First consider the following chain of isomorphisms:

$$
\begin{aligned}
& \pi_{2}^{s}(B \Gamma)=\pi_{2}\left(\Omega^{\infty} S^{\infty}(B \Gamma)\right) \cong \pi_{1}\left(\Omega \Omega^{\infty} S^{\infty}(B \Gamma)\right) \\
& \cong \pi_{1}\left(\Omega_{0} \Omega^{\infty} S^{\infty}(B \Gamma)\right) \stackrel{h}{\longrightarrow} H_{1}\left(\Omega_{0} \Omega^{\infty} S^{\infty}(B \Gamma)\right)
\end{aligned}
$$

Here, $\Omega_{0} \Omega^{\infty} S^{\infty}(B \Gamma)$ denotes the component of the constant loop, and $h$ is the Hurewicz homomorphism, which is an isomorphism since $\Omega_{0} \Omega^{\infty} S^{\infty}(B \Gamma)$ is pathconnected and $\pi_{1}$ is abelian (since it is actually $\pi_{2}$ of a space).

On the other hand, by Theorem 2,

$$
H S_{1}(k[\Gamma]) \cong H_{1}\left(\Omega \Omega^{\infty} S^{\infty}(B \Gamma) ; k\right) \cong H_{1}\left(\Omega \Omega^{\infty} S^{\infty}(B \Gamma)\right) \otimes k .
$$

Note, all tensor products will be over $\mathbb{Z}$ in this section. Now $\Omega \Omega^{\infty} S^{\infty}(B \Gamma)$ consists of disjoint homeomorphic copies of $\Omega_{0} \Omega^{\infty} S^{\infty}(B \Gamma)$, one for each element of $\Gamma /[\Gamma, \Gamma]$, (where $[\Gamma, \Gamma]$ is the commutator subgroup of $\Gamma$ ), so we may write

$$
H_{1}\left(\Omega \Omega^{\infty} S^{\infty}(B \Gamma)\right) \otimes k \cong H_{1}\left(\Omega_{0} \Omega^{\infty} S^{\infty}(B \Gamma)\right) \otimes k[\Gamma /[\Gamma, \Gamma]]
$$

to obtain the following result:

Proposition 95 If $\Gamma$ is a group, then $H S_{1}(k[\Gamma]) \cong \pi_{2}^{s}(B \Gamma) \otimes k[\Gamma /[\Gamma, \Gamma]]$.

As an immediate corollary, if $\Gamma$ is abelian, then $H S_{1}(k[\Gamma]) \cong \pi_{2}^{s}(B \Gamma) \otimes k[\Gamma]$. Moreover, by results of Brown and Loday [2], if $\Gamma$ is abelian, then $\pi_{2}^{s}(B \Gamma)$ is the reduced tensor square, $\Gamma \widetilde{\wedge}=(\Gamma \otimes \Gamma) / \approx$, where $g \otimes h \approx-h \otimes g$ for all $g, h \in \Gamma$. 


\section{Proposition 96}

$$
H S_{1}\left(k\left[C_{n}\right]\right)= \begin{cases}(\mathbb{Z} / 2 \mathbb{Z})^{n} & n \text { even } \\ 0 & n \text { odd }\end{cases}
$$

Proof $\pi_{2}^{s}\left(B C_{n}\right)=\mathbb{Z} / 2 \mathbb{Z}$ if $n$ is even, and 0 if $n$ is odd. The result then follows from Proposition 95, as $k\left[C_{n} /\left[C_{n}, C_{n}\right]\right] \cong k\left[C_{n}\right] \cong k^{n}$, as $k$-module.

\section{Relations to cyclic homology}

The relation between the symmetric bar construction and the cyclic bar construction arising from the inclusions $\Delta C \hookrightarrow \Delta S$ gives rise to a natural map $H C_{*}(A) \rightarrow$ $H S_{*}(A)$ Indeed, by Remark 17, we may define cyclic homology thus: $H C_{*}(A)=$ $\operatorname{Tor}_{*}^{\Delta C}\left(\underline{k}, B_{*}^{\mathrm{sym}} A\right)$, where we understand $B_{*}^{\mathrm{sym}} A$ as the restriction of the functor to $\Delta C$.

Using the partial complex of Theorem 5, and an analogous one for computing cyclic homology (see Loday [16, page 59]), the map $H C_{*}(A) \rightarrow H S_{*}(A)$ for degrees 0 and 1 is induced by the following partial chain map:

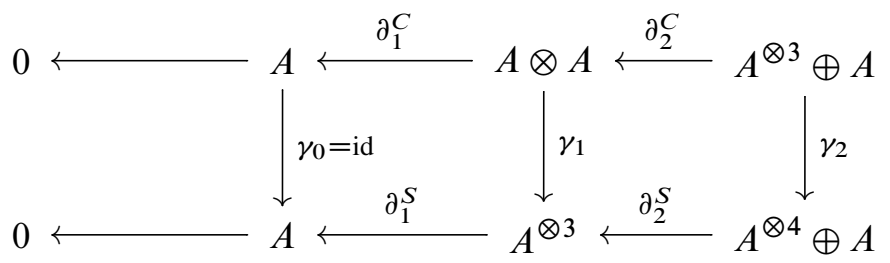

In this diagram, the boundary maps in the upper row are defined as follows:

$$
\begin{aligned}
& \partial_{1}^{C}: a \otimes b \mapsto a b-b a \\
& \partial_{2}^{C}: \begin{cases}a \otimes b \otimes c & \mapsto a b \otimes c-a \otimes b c+c a \otimes b \\
a & \mapsto 1 \otimes a-a \otimes 1\end{cases}
\end{aligned}
$$

The boundary maps in the lower row are defined as in Theorem 5.

$\partial_{1}^{S}: a \otimes b \otimes c \mapsto a b c-c b a$

$\partial_{2}^{S}: \begin{cases}a \otimes b \otimes c \otimes d & \mapsto a b \otimes c \otimes d-d \otimes c a \otimes b+b c a \otimes 1 \otimes d+d \otimes b c \otimes a \\ a & \mapsto 1 \otimes a \otimes 1\end{cases}$ 
The partial chain map is given in degree 1 by $\gamma_{1}(a \otimes b) \stackrel{\text { def }}{=} a \otimes b \otimes 1$. In degree $2, \gamma_{2}$ is defined on the summand $A^{\otimes 3}$ via

$a \otimes b \otimes c \mapsto(a \otimes b \otimes c \otimes 1-1 \otimes a \otimes b c \otimes 1+1 \otimes c a \otimes b \otimes 1$

$$
+1 \otimes 1 \otimes a b c \otimes 1-b \otimes c a \otimes 1 \otimes 1,-2 a b c-c a b),
$$

and on the summand $A$ via

$$
a \mapsto(-1 \otimes 1 \otimes a \otimes 1,4 a)
$$

\subsection{Examples}

To provide some examples, consider the maps $\gamma_{1}: H C_{1}\left(\mathbb{Z}[t] /\left(t^{n}\right)\right) \rightarrow H S_{1}\left(\mathbb{Z}[t] /\left(t^{n}\right)\right)$. It can be shown (for example, by direct computation) that $H C_{1}\left(\mathbb{Z}[t] /\left(t^{2}\right)\right) \cong \mathbb{Z} / 2 \mathbb{Z}$ is generated by the $1-$ chain $t \otimes t \cdot \gamma_{1}(t \otimes t)=t \otimes t \otimes 1 \in H S_{1}\left(\mathbb{Z}[t] /\left(t^{2}\right)\right.$ is a non-trivial element of $\mathbb{Z} / 2 \mathbb{Z} \oplus \mathbb{Z} / 2 \mathbb{Z}$ (which may be verified by direct computation as well).

The map $H C_{1}\left(\mathbb{Z}[t] /\left(t^{3}\right)\right) \rightarrow H S_{1}\left(\mathbb{Z}[t] /\left(t^{2}\right)\right)$ may be similarly analyzed. Here, the chain $t \otimes t+t \otimes t^{2}$ is a generator of $H C_{1}\left(\mathbb{Z}[t] /\left(t^{3}\right)\right) \cong \mathbb{Z} / 6 \mathbb{Z}$, which gets sent by $\gamma_{1}$ to $t \otimes t \otimes 1+t \otimes t^{2} \otimes 1$, a non-trivial element of $H S_{1}\left(\mathbb{Z}[t] /\left(t^{3}\right)\right) \cong \mathbb{Z} / 2 \mathbb{Z} \oplus \mathbb{Z} / 2 \mathbb{Z}$.

The case $n=4$ is bit more interesting. Here, $H C_{1}\left(\mathbb{Z}[t] /\left(t^{4}\right)\right) \cong \mathbb{Z} / 2 \mathbb{Z} \oplus \mathbb{Z} / 12 \mathbb{Z}$, generated by $t \otimes t$ and $t \otimes t^{2}+t \otimes t^{3}$, respectively. The image of the map $\gamma_{1}$ in $H S_{1}\left(\mathbb{Z}[t] /\left(t^{4}\right)\right)$ is $(\mathbb{Z} / 2 \mathbb{Z})^{2} \subseteq(\mathbb{Z} / 2 \mathbb{Z})^{4}$.

\section{Using computer algebra systems for computing symmet- ric homology}

The computer algebra systems GAP, Octave and Fermat were used to verify proposed theorems and also to obtain some concrete computations of symmetric homology for some small algebras. A tar-file of the scripts that were created and used for this work is available at http://arxiv.org/e-print/0807.4521v1/. This tar-file contains the following files:

- Basic.g - Some elementary functions, necessary for some functions in Deltas.g

- HomAlg.g - Homological Algebra functions, such as computation of homology groups for chain complexes.

- Fermat.g - Functions necessary to invoke Fermat for fast sparse matrix computations. 
- fermattogap, gaptofermat - Auxiliary text files for use when invoking Fermat from GAP.

- DeltaS.g - This is the main repository of scripts used to compute various quantities associated with the category $\Delta S$, including $H S_{1}(A)$ for finitedimensional algebras $A$.

In order to use the functions of DeltaS.g, simply copy the above files into the working directory (such as $\sim / g a p /$ ), invoke GAP, then read in DeltaS.g at the prompt. The dependent modules will automatically be loaded (hence they must be present in the same directory as DeltaS.g). Note that most of the computations involving homology require substantial memory to run. I recommend calling GAP with the command line option "-o mem", where mem is the amount of memory to be allocated to this instance of GAP. All computations done in this dissertation can be accomplished by allocating 20 gigabytes of memory. The following provides a few examples of using the functions of DeltaS.g

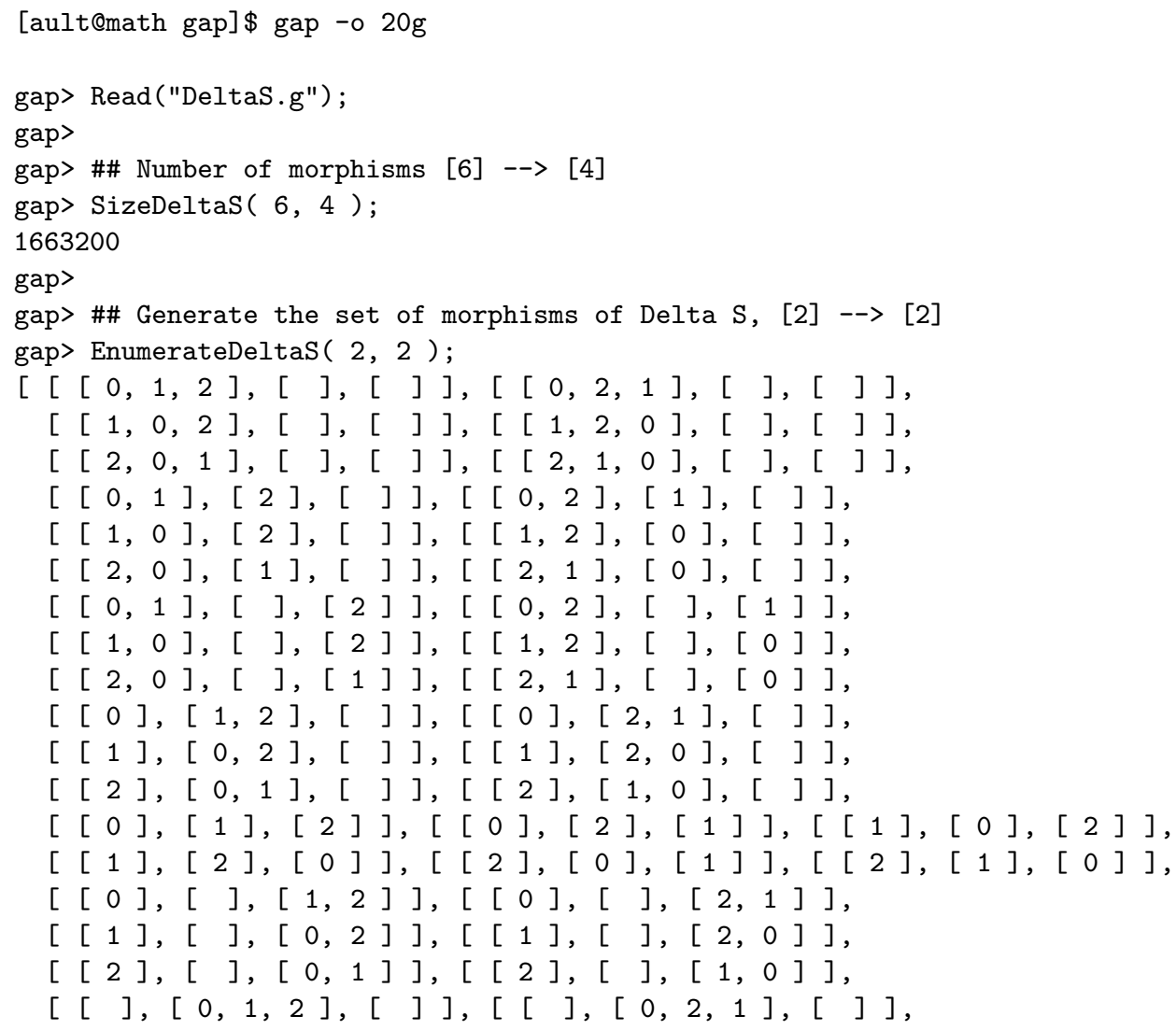


$[[],[1,0,2],[]],[[],[1,2,0],[]]$,

$[[],[2,0,1],[]],[[],[2,1,0],[]]$,

$[[],[0,1],[2]],[[],[0,2],[1]]$,

$[[],[1,0],[2]],[[],[1,2],[0]]$,

$[[],[2,0],[1]],[[],[2,1],[0]]$,

$[[],[0],[1,2]],[[],[0],[2,1]]$,

$[[],[1],[0,2]],[[],[1],[2,0]]$,

$[[],[2],[0,1]],[[],[2],[1,0]]$,

$[[],[],[0,1,2]],[[],[],[0,2,1]]$,

$[[],[],[1,0,2]],[[],[],[1,2,0]]$,

$[[],[],[2,0,1]],[[],[],[2,1,0]]]$

\section{gap>}

gap> \#\# Generate only the epimorphisms [2] --> [2]

gap> EnumerateDeltaS ( 2, 2 : epi );

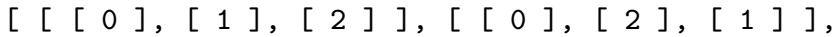

$\left[\left[\begin{array}{l}1 \\ ]\end{array},[0],[2]\right],\left[\left[\begin{array}{ll}1 & 0\end{array}\right],[2],[0]\right]\right.$,

$\left[\left[\begin{array}{l}2 \\ {[}\end{array},[0],[1]\right],[[2],[1],[0]]\right]$

\section{gap>}

gap> \#\# Compose two morphisms of Delta S.

gap> a := Random(EnumerateDeltaS $(4,3))$;

[ [ 0$],[2,4,1],[],[3]]$

gap $>b:=$ Random (EnumerateDeltaS $(3,2))$;

[ [ ], [ $3,0,2],[1]]$

gap> MultDeltaS $(b, a)$;

$[[],[3,0],[2,4,1]]$

gap> MultDeltaS(a, b);

Maps incomposeable

[ ]

gap>

gap> \#\# Examples of using morphisms of Delta $\mathrm{S}$ to act on simple tensors

gap> A := TruncPolyAlg $([3,2])$;

<algebra of dimension 6 over Rationals>

gap> \#\# TruncPolyAlg is defined in Basic.g

gap> \#\# TruncPolyAlg $\left(\left[i_{-} 1, i_{-} 2, \ldots, i_{-} n\right]\right)$ is generated by

gap> \#\# $x_{-} 1, x_{-} 2, \ldots, x_{-} n$, under the relation $\left(x_{-} j\right)-\left(i_{-} j\right)=0$.

gap> $g:=$ GeneratorsOfLeftModule $(A)$;

$\left[X^{\wedge}[0,0], X^{\wedge}[0,1], X^{\wedge}[1,0], X^{-}[1,1], X^{\wedge}[2,0], X^{\wedge}[2,1]\right]$

gap> $\mathrm{x}:=\mathrm{g}[2] ; \mathrm{y}:=\mathrm{g}[3]$;

$\mathrm{X}^{\wedge}[0,1]$

$\mathrm{X}^{\sim}[1,0]$

gap $>\mathrm{v}:=\left[\mathrm{x} * \mathrm{y}, 1, \mathrm{y}^{\wedge} 2\right]$;

gap> \#\# v represents the simple tensor xy lotimes 1 lotimes $\mathrm{y}^{\wedge} 2$.

$\left[X^{\wedge}[1,1], 1, X^{\wedge}[2,0]\right]$

gap> ActByDeltaS ( v, [[2], [], [0], [1] ] );

$\left[X^{\wedge}[2,0], 1, X^{-}[1,1], 1\right]$

gap> ActByDeltaS( v, [[2], [0,1]] );

$\left[\mathrm{X}^{-}[2,0], \mathrm{X}^{-}[1,1]\right]$

gap> $\operatorname{ActByDeltaS}(\mathrm{v},[[2,0],[1]])$; 
$\left[0 * \mathrm{X}^{\wedge}[0,0], 1\right]$

gap>

gap> \#\# Symmetric monoidal product on DeltaS_+

gap> a := Random(EnumerateDeltaS $(4,2))$;

$[$ [ ], [ 2, 1, 0], [ 3,4 ] ]

gap $>\mathrm{b}:=$ Random(EnumerateDeltaS $(3,3))$;

[ [ ], [ ], [ ], [ $1,3,2,0]]$

gap> MonoidProductDeltaS $(\mathrm{a}, \mathrm{b})$;

$[[],[2,1,0],[3,4],[],[],[],[6,8,7,5]]$

gap> MonoidProductDeltaS $(b, a)$;

$[[],[],[],[1,3,2,0],[],[6,5,4],[7,8]]$

gap> MonoidProductDeltaS (a, [] );

$[[],[2,1,0],[3,4]]$

gap>

gap> \#\# Symmetric Homology of the algebra A, in degrees 0 and 1.

gap> SymHomUnitalAlg $(A)$;

$[[0,0,0,0,0,0],[2,2,2,2,2,2,2,2,2,2,2,6,0,0]]$

gap> \#\# 'O' represents a factor of $\mathrm{Z}$, while a non-zero $\mathrm{p}$ represents

gap> \#\# a factor of $\mathrm{Z} / \mathrm{pZ}$.

gap>

gap> \#\# Using layers to compute symmetric homology

gap> C2 := CyclicGroup(2);

<pc group of size 2 with 1 generators $>$

gap> A := GroupRing(Rationals, DirectProduct(C2, C2));

<algebra-with-one over Rationals, with 2 generators>

gap> \#\# First, a direct computation without layers:

gap> SymHomUnitalAlg $(A)$;

$[[0,0,0,0],[2,2,2,2,2,2,2,2,2,2,2,2]]$

gap> \#\# Next, compute HS_O(A)_u and HS_1(A)_u for each generator $u$.

gap $>\mathrm{g}:=$ GeneratorsOfLeftModule $(\mathrm{A})$;

[ (1) $*<$ identity> of . ., (1) $* \mathrm{f} 2,(1) * \mathrm{f} 1,(1) * \mathrm{f} 1 * \mathrm{f} 2]$

gap> SymHomUnitalAlgLayered (A, g[1]);

$[$ [ 0$],[2,2,2]]$

gap> SymHomUnitalAlgLayered (A, g[2]);

[ [ 0$],[2,2,2]]$

gap> SymHomUnitalAlgLayered (A, g[3]);

$[[0],[2,2,2]]$

gap> SymHomUnitalAlgLayered (A, g[4]);

[ [ 0 ], [ $2,2,2]]$

gap> \#\# Computing HS_1( Z[t] ) by layers:

gap> SymHomFreeMonoid $(0,10)$;

HS_1 $1 k[t]) \_\left\{t^{-0} 0\right\}:$ [ ]

$\mathrm{HS}_{-} 1(\mathrm{k}[\mathrm{t}])_{-}\left\{\mathrm{t}^{-1}\right\}:$ : [ ]

$\mathrm{HS}_{-} 1(\mathrm{k}[\mathrm{t}])_{-}\left\{\mathrm{t}^{\wedge} 2\right\}:$ : [ 2$]$

$\mathrm{HS}_{-} 1(\mathrm{k}[\mathrm{t}])_{-}\{\mathrm{t}-3\}:[2]$

$\mathrm{HS}_{-} 1(\mathrm{k}[\mathrm{t}])_{-}\{\mathrm{t} \sim 4\}:$ [ 2$]$

$\mathrm{HS}_{-} 1(\mathrm{k}[\mathrm{t}])_{-}\{\mathrm{t} \sim 5\}:$ [ 2$]$

HS_1 $1 k[t])_{-}\left\{\mathrm{t}^{\wedge} 6\right\}:$ : [ 2$]$ 


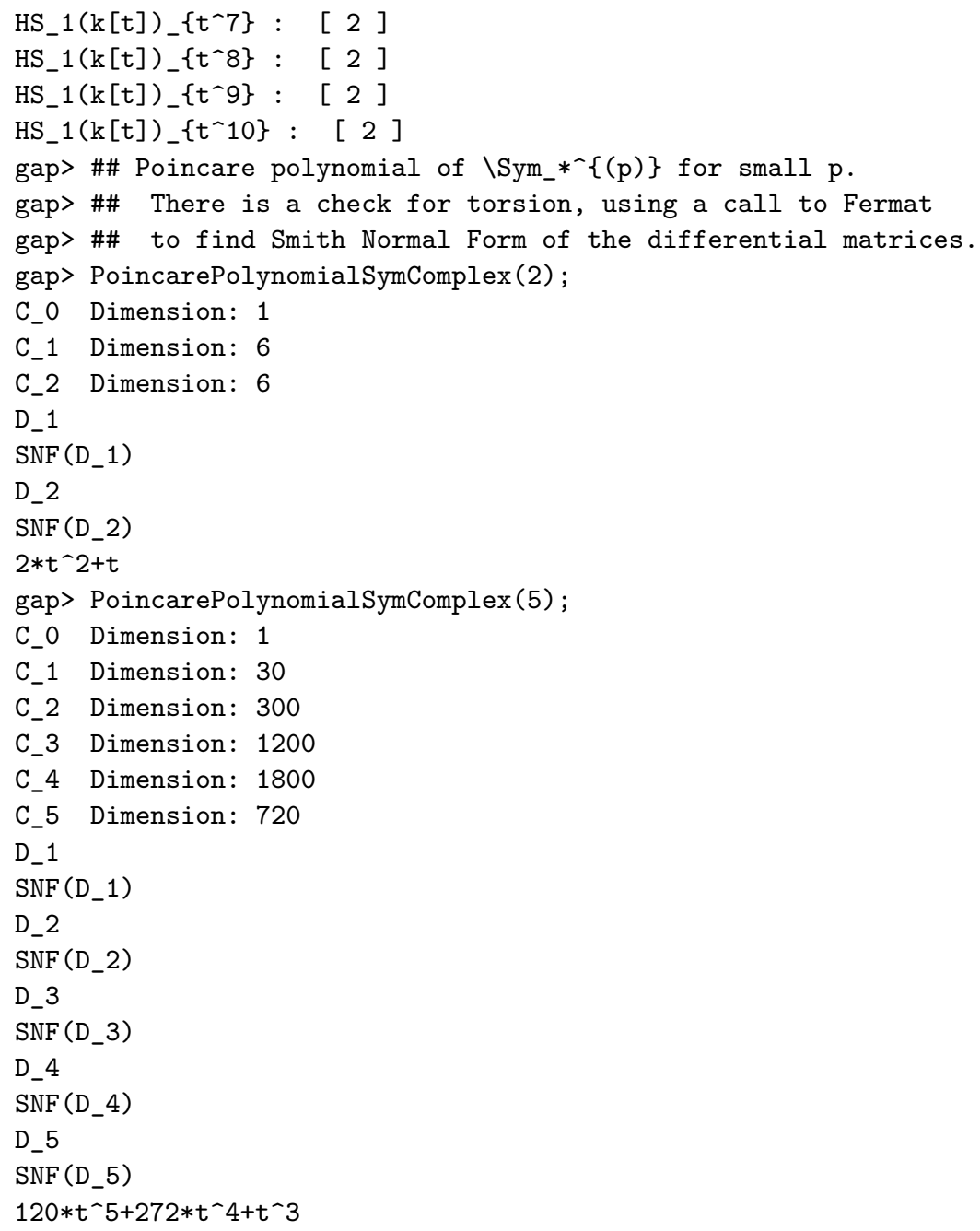

\section{References}

[1] KS Brown, Cohomology of groups, Graduate Texts in Mathematics 87, Springer, New York (1982) MR672956

[2] R Brown, J-L Loday, Van Kampen theorems for diagrams of spaces, Topology 26 (1987) 311-335 MR899052 With an appendix by M Zisman

[3] R R Bruner, J P May, J E McClure, M Steinberger, $H_{\infty}$ ring spectra and their applications, Lecture Notes in Mathematics 1176, Springer, Berlin (1986) MR836132

[4] F R Cohen, T J Lada, J P May, The homology of iterated loop spaces, Lecture Notes in Mathematics 533, Springer, Berlin (1976) MR0436146 
[5] F R Cohen, F P Peterson, On the homology of certain spaces looped beyond their connectivity, Israel J. Math. 66 (1989) 105-131 MR1017156

[6] A Dold, Universelle Koeffizienten, Math. Z. 80 (1962) 63-88 MR0140558

[7] J W Eaton, GNU Octave Manual (2002) Available at http://www.gnu.org/ software/octave/doc/interpreter/

[8] Z Fiedorowicz, The symmetric bar construction, preprint Available at http:// www.math.ohio-state.edu/ fiedorow/

[9] Z Fiedorowicz, Classifying spaces of topological monoids and categories, Amer. J. Math. 106 (1984) 301-350 MR737777

[10] Z Fiedorowicz, J-L Loday, Crossed simplicial groups and their associated homology, Trans. Amer. Math. Soc. 326 (1991) 57-87 MR998125

[11] P Gabriel, M Zisman, Calculus of fractions and homotopy theory, Ergebnisse der Mathematik und ihrer Grenzgebiete 35, Springer New York, New York (1967) MR0210125

[12] T G Group, GAP - Groups, Algorithms and Programming, version 4.4.10 (2007) Available at http://www.gap-system.org/

[13] K Itô, Encyclopedic Dictionary of Mathematics (1993)

[14] D M Kan, Adjoint functors, Trans. Amer. Math. Soc. 87 (1958) 294-329 MR0131451

[15] RH Lewis, Fermat computer algebra system (2008) Available at http:// home. bway .net/lewis

[16] J-L Loday, Cyclic homology, second edition, Grundlehren der Mathematischen Wissenschaften 301, Springer, Berlin (1998) MR1600246 Appendix E by María O Ronco, Chapter 13 by the author in collaboration with Teimuraz Pirashvili

[17] S Mac Lane, The Milgram bar construction as a tensor product of functors, from: "The Steenrod Algebra and its Applications (Proc. Conf. to Celebrate N. E. Steenrod's Sixtieth Birthday, Battelle Memorial Inst., Columbus, Ohio,1970)", Lecture Notes in Mathematics 168, Springer, Berlin (1970) 135-152 MR0273618

[18] S MacLane, Categories for the working mathematician, Graduate Texts in Mathematics 5, Springer, New York (1971) MR0354798

[19] J P May, The geometry of iterated loop spaces, Lectures Notes in Mathematics 271, Springer, Berlin (1972) MR0420610

[20] J P May, R Thomason, The uniqueness of infinite loop space machines, Topology 17 (1978) 205-224 MR508885

[21] J McCleary, A user's guide to spectral sequences, second edition, Cambridge Studies in Advanced Mathematics 58, Cambridge University Press, Cambridge (2001) MR1793722 
[22] T Pirashvili, On the PROP corresponding to bialgebras, Cah. Topol. Géom. Différ. Catég. 43 (2002) 221-239 MR1928233

[23] T Pirashvili, B Richter, Hochschild and cyclic homology via functor homology, $K-$ Theory 25 (2002) 39-49 MR1899698

[24] D Quillen, Higher algebraic $K$-theory I, from: "Algebraic $K$-theory, I: Higher $K-$ theories (Proc. Conf., Battelle Memorial Inst., Seattle, Wash., 1972)", Springer, Berlin (1973) 85-147. Lecture Notes in Math., Vol. 341 MR0338129

[25] J J Rotman, An introduction to homological algebra, Pure and Applied Mathematics 85, Academic Press [Harcourt Brace Jovanovich Publishers], New York (1979) MR538169

[26] G Segal, Classifying spaces and spectral sequences, Inst. Hautes Études Sci. Publ. Math. (1968) 105-112 MR0232393

[27] J Słomińska, Homotopy colimits on E-I-categories, from: "Algebraic topology Poznań 1989”, Lecture Notes in Math. 1474, Springer, Berlin (1991) 273-294 MR1133907

[28] B Stenström, Rings of quotients, Die Grundlehren der Mathematischen Wissenschaften 217, Springer, New York (1975) MR0389953

[29] R W Thomason, Uniqueness of delooping machines, Duke Math. J. 46 (1979) 217-252 MR534053

[30] S T Vrećica, R T Živaljević, Cycle-free chessboard complexes and symmetric homology of algebras, European J. Combin. 30 (2009) 542-554 MR2489249

Department of Mathematics, Fordham University

Bronx NY 10461, USA

ault@fordham.edu

http://fordham.academia.edu/ShaunAult/

Received: 6 January 2010 